\title{
Analysis of Soil and Water at the Four Mile Creek Seepline near the F\&H Areas of SRS (U)
}<smiles>C#CC1CCCC1</smiles>

June 20, 1990

J. S. Haselow, Environmental Sciences

M. Harris, Waste Managemerit Technology

B. B. Looney, Environniental Sciences

N. V. Halverson, Environmental Sciences (formerly)

J. B. Gladden, Envirorımental Sciences

Approved By:

D. B. Moore, Section Manager Environmental Sciences

\section{DISCLAIMER}

This report was prepared as an account of work sponsored by an agency of the United States Government. Neither the United States Government nor any agency thereof, nor any of their employees, makes any warranty, express or implied, or assumes any legal liability or responsibility for the accuracy, completeness, or usefulness of any information, apparatus, product, or process disclosed, or represents that its use would not infringe privately owned rights. Reference herein to any specific commercial product, process, or service by trade name, trademark, manufacturer, or otherwise does not necessarily constitute or imply its endorsement, recommendation, or favoring by the United States Government or any agency thereof. The views and opinions of authors expressed herein do not necessarily state or reflect those of the United States Government or any agency thereof.

WESTINGHOUSE SAVANNAH RIVER COMPANY

SAVANNAH RIVER LABORATORY

SAVANNAH RIVER SITE

AIKEN, SC 29808

Prepared for the U. S. Department of Energy under Contract No. DE-ACO9-8ASR18035 
WSRC-RP-90-0591

\section{Executive summary}

Several soil and water samples wexe collected along the Four Mile Creek (FMC) seepline at the F \& H Areas of the Savannah River Site. The samples were analyzed for concentrations of metals, radionuclides, and inorganic constituents. The results of the analyses are summarized below for the soil and water samples.

Soil

Soil concentrations were compared to values obtained from a survey of unimpacted soils on the SRS (Looney et al., 1989).

Cadmium is the only metal that exceeds the maximum sRs background soil value (1.19 ppm), and that was only for one sample out twenty-six. Gross beta exceeds the background value (23 pCi/g) four out of twenty-six times; ${ }^{90} \mathrm{Sr}$ exceeded the maximum background value $(<1 \mathrm{pci} / \mathrm{g})$ six out of twerty-six times, and ${ }^{233 / 234} \mathrm{U}$ and ${ }^{238} \mathrm{U}$ each exceeded the maximum background value for total uranium $(4.7 \mathrm{pCi} / \mathrm{g})$ one out of twenty-six times.

${ }^{249} \mathrm{Am},{ }^{244} \mathrm{Cm},{ }^{60} \mathrm{Co},{ }^{129} \mathrm{I},{ }^{238} \mathrm{Pu}$, and ${ }^{226} \mathrm{Ra}$ may also be above background activities at least in one sample. However, because there are no available background data to compare the results, this conclusion is based on: 1) an apparent correlation with other impacted soil samples (high values of other radionuclides such as ${ }^{90} \mathrm{Sr}$ ), 2) comparison with soil samples that are out of the central portion of the plume, and 3) knowledge of constituents that have potentially been released into the basins in the past.

Water

Water concentrations were compared to proposed or established drinking water standards.

All of the metals concentrations are below the Primary Drinking Water standard at the FMC seepline except for cadmium in F-Area. Measured cadmium concentrations were slightly above the proposed standard of $5 \mathrm{ppb}$ (but not the established standard of $10 \mathrm{ppb}$ ) during both the 1988 and 1989 sampling period at one point in the central portion of the plume from the basins. Manganese and iron concentrations are above the Secondary Drinking water standard at both the $F$ \& $H$ Area seepline. Aluminum and sodium concentrations are apparently elevated, but no standards exist for these constituents. The sodium is probably from the caustic discharged to the basins, and the aluminum is probably being leached from the soil matrix (Looney et al., 1988).

Gross alpha, gross beta, ${ }^{241} \mathrm{Arn},{ }^{129} \mathrm{I},{ }^{3} \mathrm{H},{ }^{226} \mathrm{Ra},{ }^{89} \mathrm{Sr},{ }^{90} \mathrm{Sr},{ }^{233 / 234} \mathrm{U}$, and ${ }^{238} U$ are above either the proposed or established drinking 
water standard at one point or more along the F-Area FMC seepline. Gross alpha, gross beta, ${ }^{3} \mathrm{H},{ }^{226} \mathrm{Ra},{ }^{89} \mathrm{Sr}$, and ${ }^{90} \mathrm{Sr}$ are above either the proposed or established drinking water standard at one point or more along the H-Area FMC seepline. ${ }^{244} \mathrm{Cm},{ }^{60} \mathrm{Co}$, ${ }^{134} \mathrm{Cs}$ and ${ }^{137} \mathrm{Cs}$ have apparently impacted the seepline water as indicated by the elevated concentrations of these constituents in the central portion of the plume as compared to the outer edges. Gross beta and ${ }^{3} \mathrm{H}$ are above either the proposed or established drinking water standard at one point or more in $\mathrm{FMC}$.

Nitrate is also above the PDWS (10 ppm) at both the $F$ \& $H$ Area seepline, but not in the creek. 
Table of Contents

Page

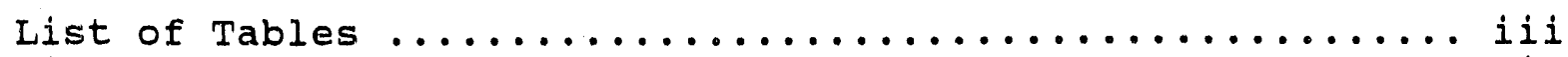

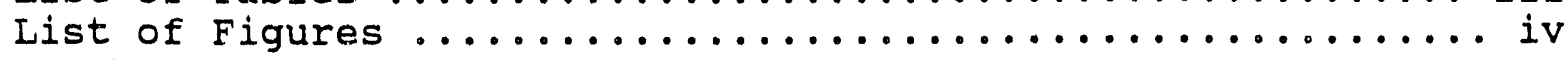

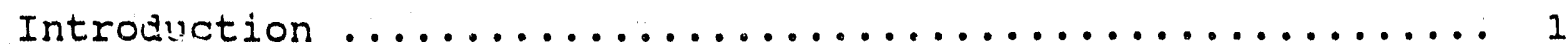

Extensive Seepline Survey for $\mathrm{pH}$,

Conductivity and Tritiun ...................... 2

Extended Chemical Analysis .....................

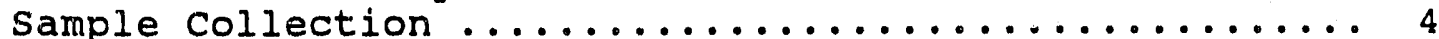

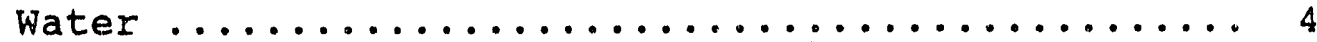

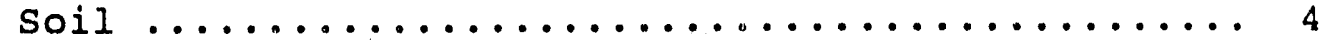

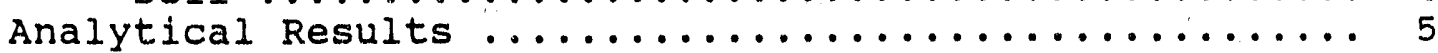

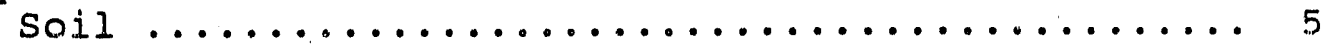

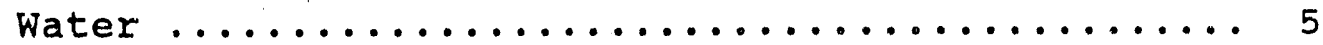

References $\ldots \ldots \ldots \ldots \ldots \ldots \ldots \ldots \ldots \ldots \ldots \ldots \ldots \ldots \ldots \ldots$

Tables

Figures

Appendices 
WSRC-RP-90-0591

\section{List of Tables}

Table 1. Analyses completed for 1988 ant 1989 soil and water samples.

Table 2. Tritium concentrations and conductivities of Four Mile Creek Seepline water.

Table 3. 1988 and 1989 Four Mile Creek Seepline Soil Results Metals.

Table 4. 1988 and 1989 Four Mile creek Seepline Soil Results Radionuclides.

Table 5. 1988 seepline Soil Chloride and Nitrate Concentrations

Table 6. 1988 and 1989 Four Mile creek seepline and Stream Water Samples - Metals

Table 7. 1988 and 1989 Four Mile cretk seepline and Stream Water Samples - Radionuclides

Table 8. 1988 and 1989 Four Mile creek Seepline and Stream Water Sampiles - Miscellaneous Parameters 


\section{List of Figures}

Figure 1. Location of the Separations Area.

Figure 2. Sampline Points for F-Area Tritium Survey.

Figure 3. Sampline Points for H-Area Tritium Survey.

Figure 4. F-Area tritium plume at the Four Mile creek seepline.

Figure 5. H-Area tritium plume at the Four Mile creek seeksine.

Figure 6. F\&H Area tritium plume at the Four Mile Creek seepline.

Figure 7. F\&H Area conductivity plume at the Four Mile Creek seepline.

Figure 8. Location of vibracore samples collected in 1988.

Figure 9. Location of vibracore samples collected in 1989.

Figure 10. Location of seepline water samples collected in 1988 .

Figure 11. Location of seepline watex samples collected in 1989. 


\section{INTRODUCTION}

Until 1988, solutions containing sodium hydxoxide, nitric acid, low levels of radionuclides (mostly tritiated water) and some metals were discharged to unlined seepage basins at the $F$ and $H$ Areas of the Savannah River Site (SRS) as part of normal operations (Killian et al, 1987a,b). The basins are now being closed according to the Resource conservation and Recovery Act (RCRA). As part of the closure, a Part B Post-Closure Care Permit is being prepared. The information included in this report will fulfill some of the data requirements for that part $B$ permit.

The area that includes the basins is bounded to the north and west by Upper Three Runs Creek, and to the south by Four Mile Creek (Figure 1). These tributaries of the Savannah River are drains for the shallow groundwater system in the area. Constituents entering the basins seep to the underlying water table. Once in the water table, most of the constituents flow horizontally to the south in the water table towards Four Mile creek (FMC). However, a relatively small fraction of the constituents enter a lower water-bearing formation that flows towards Upper Three Runs creek. Numerical simulations of flow in the hydrciogic system undexlying the basins indicate that travel times for unretarded constituents from the basins to FMC is on the order of 10 years, and about 70 years from the basins to Upper Three Runs Creek (GeoTrans, 1988). Discharges to the basins started in the 1950's. Therefore, a steady-state profile for unretarded constituents (e.g. tritium) has developed between the basins and FMC. It is unlikely that constituents emanating from the basins have reached Upper Three Runs creek.

FMC is bordered by well developed floodplains and wetland areas. Consequently, a portion of the groundwater moving downgradient from the basins to FMC reaches the surface prior to entering the stream. This downslope region of saturated or near-saturated surface soils is most easily distinguished by a transition from upland to wetland type vegetation (conifers to bottomland hardwoods), and this transition zone is designated in this report as the seepline.

The goal of the work reported herein is to document the impacts from the basins on FMC and the wetlands adjacent to FMC. The study of FMC has been completed in a phased approach. Looney et al. (1988) reported the results of a preliminary nonradionuclide study to determine impacts to the area. Then in the fall of 1988, soil cores and water samples were taken along the FMC seepline and analyzed for both nonradioactive and radioactive constituents listed in Table?. This study was followed by an extensive survey along the FMC seepline where 
tritium was measured at over three hundred points. Because tritiated water essentially moves as untritiated water, this study provides a detailed delineation of the size and nature of the impacted groundwater. In the late summer of 1989 , water and soil samples were again taken at points along FMC. The list of constituents analyzed during this sampling period are also listed in Table 1. The 1988 soil and water sampling period followed a below normal precipitation period, and the 1989 sampling period followed an above normal precipitation period. Several other constituent specific studies have been completed along FMC in the area in both the 1960's and 1970's. These studies are given as references in Looney et al., 1988, which is given as Appendix A.

\section{Extensive seepline survey for pH, conductivity and Tritium}

During March, 1989, seepline water samples were taken at over three hundred points along the FMC. The samples were collected by excavating a cylindrical plug of soil about ten inches deep and with a diameter of about five inches at a nearly saturated zone along FMC. Water was allowed to seep into the excavated space, and a twenty milliliter sample was collected. The water sample was analyzed for conductivity in the field, and for tritium at a $Q A$ level 1 laboratory. Additional samples were collected using the same procedure during January, 1990 to complete the seepline study. All sampling locations are shown on Figures 2 and 3 (foldout maps at the end).

During the 1990 sampling for tritium, sixteen duplicate samples were collected (HSP-94 to HSP-109) to determine whether the concentration of tritium was changing substantially along the seepline (Table 2). A statistical analysis was completed using paired differences (Appendix B). The statistical analysis does not allow one to reject the hypothesis that the tritium concentrations are equivalent, even though nine out of sixteen recent samples have greater tritium concentrations. Ostensibly, the variance in the data is too great to statistically determine a difference. As a result, tritium and conductivity data obtained during the 1889 and 1990 sampling period are presented together.

The complete results of the tritium seepline survey are given in Table 2. Concentrat cons of tritium in the seepline water ranged from below the analytical detection limit to three orders of magnitude above the primary drinking water standard (PDWS), which is $20 \mathrm{pCi} / \mathrm{ml}$. As expected, the tritium results provide excellent evidence of the extent of the plume from the basins. The results also delineate regions of preferential subsurface flow. This is depicted in Figures 4 and 5 where the concentration of tritium along the seepline is represented as proportional to the size of 
the shaded circles for F-Area and $\mathrm{H}-\mathrm{Area}$, respectively. A pattern of larger circles denotes the areas of preferential flow. Also shown in these figures is that the extent of the plume falls off exponentially from the center of the plume. Evidence of preferential flow exists even away from the center of the plume.

Figure 6 shows the tritium concentrations for the entire area. The tritium concentrations increase downgradient from the $F \& H$ Area seepage basins and from the old Burial Ground. The information in this report is intended for the F\&H Basins closure, but the old Burial Grounds may also influence both the surface and subsurface water chemistry in the vicinity.

The results of the field-measured specific conductivity are given in Table 2 and are illustrated in Figure 7 . The measured conductivities are influenced by the soil and water chemistry at the point of sampling. Nevertheless, the conductivity data correlate fairly well with the tritium concentrations (elevated tritium with elevated conductivity) except for a few samples in the vicinity of influence of the old Burial Ground. This is because the old Burial Ground received discharges containing tritium, but not nearly the volume of ions (salts) that the basins did. Thus, the conductivities directly downgradient from the old Burial Ground are relatively low even though some tritium concentrations are above the PDWS.

The $\mathrm{pH}$ varies along the seepline from 3.7 to 6.5 with an average value of 4.90 (Appendix $C$ ). The $\mathrm{pH}$ has been reduced because of the nitric acid that was discharged to the basins. The acidity of the groundwater influences the observed metals concentrations for two reasons. The species of metals and thus the solubility is a function of the $\mathrm{pH}$, and the acid is "dissolving" the soil matrix. The former observation is well documented in the geochemical literature, and the latter observation was reported in Looney et al., 1988 (Appendix A).

\section{Extepded Chemical Analyses}

In addition to the extensive sampling for tritium, $\mathrm{pH}$, and conductivity along FMC, additional seepline water, stream water, and soil core samples were collected and analyzed in 1988 aid 1989 for the constituents 1 isted in Table 1 . The 1988 sampling was during a low precipitation period, and the 1989 sampling was during a high precipitation period. The locations of the sampling points for the 1988 soil, 1989 soil, 1988 water, and 1989 water are given in Figures $8,9,10$, and 11 , respectively. The coded locations on these figures correspond to those given in the tables of this document, but in the tables the year proceeds the code. Note that the there is not a direct correspondence 
WSRC-RP-90-0591

between 1988 and 1989 sampling points. For instance, 88HWI is not at the same point as $89 \mathrm{HW} 1$.

For the 1988 water samples, a stream or creek sample is denoted with a CW (creek water), and a seepline sample is denoted as a $\mathrm{HW}$ or FW. In 1989, FW and $\mathrm{HW}$ indicate whether the water sample is closest to F-Area or H-Area, but not a stream or seepline sample. The following codes are for in-stream samples: $89 \mathrm{HW}-2,89 \mathrm{HW}-7$, $89 \mathrm{HW}-8,89 \mathrm{FW}-4$. (This information is provided in the tables.)

Both the 1988 and 1989 soil samples are coded with a FS or HS (F-soil or $\mathrm{H}-\mathrm{So}$-il) and a prefix to denote the year the sample was collected. Again, there is not a direct correspondence between a 1988 sample and a 1989 sample location. Selection of 1988 soil samples for analyses was based on scans of radioactivity, and the 1989 soil samples were selected to fulfill data needs.

\section{Sample coliection}

Water

The seepline water samples were collected as described earlier. The stream water samples were collected by immersing collection containers into the stream. The water samples were preserved according to the conditions specified in DPSOP-254. For example, water samples that were analyzed for metals were kept at $4^{\circ} \mathrm{C}$, then filtered with a 45 micron filter, and then preserved with nitric acid to reduce the $\mathrm{pH}$ to below 2.0 .

\section{Soil}

Three meter-long continuous soil core samples were taken at the points listed in Figure 8 and 9. The soil cores were collected by first vibracoring a 10 centimeter diameter aluminum tube down 3 meters. This tube served as an outer casing to prevent borehole collapse after retrieval of the soil core. A 7.5 centimeter tube was then vibracored inside the outer tube.

After the inner $7.5 \mathrm{~cm}$ diameter core was in place, it was capped with a plumber's test plug, and a vacuum was applied with a hand pump to ensure recovery of the core. The inner core barrel was retrievea by utilizing a jack, tripod, and tackle arrangement. All soil samples were stored at approximately $4{ }^{\circ} \mathrm{C}$ until analysis. Soll cores were halved, and one portion has been stored for future reference.

The soil cores were separated into approximately 25 centimeter sections (10 inches) for those samples collected during 1988 . The cores collected during 1989 were separated according to 
WSRC-RP-90-0591

lithologic sections and each distinct section was coded with a letter. The soil core descriptions are given in Appendix D along with the lettering scheme.

\section{Analytical Results}

Soil

The analytical results obtained from the analyses of soil core samples are reported in Tables 3,4 , and 5 for metals, radionuclides, and inorganics, respectively. For the 1988 data, the number proceeding the colon correspond to the number of inches below the surface. For the 1989 data, letters are used to denote lithologic zones (Appendix D). To determine if the soil in the vicinity of the basins is impacted, the data were compared to results obtained from a background soil study of other unimpacted areas at the SRS (Looney et al., 1990). The soil in the vicinity of the basins is defined as impacted if it exceeds the maximum value determined from the background soil study.

Cadmium was the only metal that exceeded the maximum background value (1.19 ppm), and that was only for one sample out 26 . None of the inorganics (nitrate and chloride) exceeded the background values. Probably because these chemicals are relatively mobile, and therefore are not bound to the soil matrix. Gross beta exceeded the background value $(23 \mathrm{pCi} / \mathrm{g})$ four out of twenty-six times; ${ }^{90} \mathrm{Sr}$ exceeded the maximumbackground value $(<1 \mathrm{pCi} / \mathrm{g})$ six out of twenty-six times, and ${ }^{233 / 234} \mathrm{U}$ and ${ }^{238} \mathrm{U}$ each exceeded the maximum background value for total uranium $(4.7 \mathrm{pci} / \mathrm{g})$ one out of twenty-six times.

${ }^{241} \mathrm{Am},{ }^{244} \mathrm{Cm},{ }^{60} \mathrm{Co},{ }^{129} \mathrm{I},{ }^{238} \mathrm{Pu}$, and ${ }^{226} \mathrm{Ra}$ may also be above background values at least once for each of these constituents. However, there are no available background data to compare the results; therefore this observation is based on: 1) an apparent correlation with other impacted soil samples (high values of other radionuclides such as ${ }^{90} \mathrm{Sr}$ ), 2) comparison with soil samples that are out of the central portion of the plume, and 3 ) knowledge of constituents that have potentially been released into the basins in the past.

Water

The analytical results obtained from the analyses of seepline and stream water samples are reported in Tables 6,7 , and 8 for metals, radionuclides, and miscellaneous parameters, respectively. Federal water standards (PWDS, Secondary Drinking Water Standards (PDWS), Maximum Concentration Levels) are used 
for comparison, for constituents in which they exist. For some of the chemicals, both established and proposed standards have been published. In this case, the lower of the two values is used for comparison (see Appendix E). Also, for some constituents, there are no standards, and as a result, one can only postulate whether the concentration is elevated (indirectly or directly) from past discharges to the basins.

All of the metals concentrations are below the PDWS at the FMC seepline except for cadmium in F-Area (Table 6). Measured cadmium concentrations were slightly above the proposed standard of $5 \mathrm{ppb}$ (but not the established standard of $10 \mathrm{ppb}$ ) during both the 1988 and 1989 sampling period at one point in the central portion of the plume from the basins. Manganese and iron concentrations are above the SDWS at both the $F$ \& $H$ Area seepline. However, this standard was established to avoid staining (rust spots). Aluminum and sodium concentrations are apparently elevated, but no standards exist for these constituents. The sodium is probably from the caustic discharged to the basins, and the aluminum is probably being leached from the soil matrix (Lconey et al., 1988).

Gross alpha, gross beta, ${ }^{249} \mathrm{Am},{ }^{129} \mathrm{I},{ }^{3} \mathrm{H},{ }^{226} \mathrm{Ra},{ }^{89} \mathrm{Sr},{ }^{90} \mathrm{Sr},{ }^{233 / 234} \mathrm{U}$, and ${ }^{238} \mathrm{U}$ are above either the proposed or established drinking water standard at one point or more along the F-Area FMC seepline (Table 7). Gross alpha, gross beta, ${ }^{3} \mathrm{H},{ }^{226} \mathrm{Ra},{ }^{89} \mathrm{Sr}$, and ${ }^{90} \mathrm{Sr}$ are above either the proposed or established drinking water standard at one point or more along the H-Area FMC seepline. ${ }^{244} \mathrm{~cm},{ }^{60} \mathrm{CO}$, ${ }^{134} \mathrm{Cs}$ and ${ }^{137} \mathrm{Cs}$ have apparently impacted the seepline water as indicated by the elevated concentrations of these constituents in the central portion of the plume as compared to the outer edges. only gross beta and ${ }^{3} \mathrm{H}$ are above either the proposed or established drinking water standard at one point or more in FMC.

Nitrate is also above the PDWS (10 ppm) at both the $F$ \& $H$ Area seepline (Table 8 ), but not in the stream. However, it is at least half the PWDS in FMC in both $F \& H$ Area. The concentration of chloride is apparently unaffected by the basins, because the upstrearn sample at $89 \mathrm{HW}-8$ is about the same as along the seepline and in FMC (Table 8). 


\section{References}

GeoTrans, 1988. Characterization of Groundwater Flow and Transport in the General Separations Area Savannah River Plant, report prepared for E.J. du Pont and de Nemours, Savannah River Laboratory, Aiken, SC.

Killian, T.H., N.L. Kolb, P. Corbo, and I.W. Marine, 1987 a. Environmental Information Document: F-Area Seepage Basins. DPST-85-704, Savannah River Laboratory, Aiken, SC.

Killian, T.H., N.L. Kolb, P. Corbo, and I.W. Marine, 1987b. Environmental Information Document: H-Area Seepage Basins. DPST-85-706, Savannah River Laboratory, Aiken, SC.

Looney, B.B., J.E. Cantrell, and J.R. Cook, 1988. Sampling and Analysis of Surface water in the vicinity of the $\mathrm{F}$ - and $\mathrm{H}-$ Area Seepage Basins, DPST-88-229.

Looney, B.B., C.A. Eddy, M. Ramdeen, J. Pickett, V. Rogers, P.A. Shirley, and M.T. Scott, 1990. Geochemical and Physical Properties of Soils and Shallow sediments at the Savannah River Site, WSRC-RP-90-0464. 
Table 1. Analyses completed for 1988 and 1989 soil and water samples.

\begin{tabular}{|c|c|c|c|c|}
\hline \multirow[b]{2}{*}{ METALS } & \multicolumn{2}{|c|}{1988} & \multicolumn{2}{|c|}{1989} \\
\hline & WATER & SOIL & WATER & SOIL \\
\hline Ag & $\mathrm{X}$ & $\mathrm{X}$ & $\mathrm{X}$ & $\mathrm{X}$ \\
\hline $\mathrm{Sb}$ & $\mathrm{X}$ & $\mathrm{X}$ & & \\
\hline Al & $\mathrm{x}$ & $\mathrm{x}$ & $\mathrm{x}$ & $x$ \\
\hline As & & & $\mathrm{X}$ & $\mathrm{x}$ \\
\hline $\mathrm{Ba}$ & $\mathrm{X}$ & $\mathrm{X}$. & & \\
\hline $\mathrm{cd}$ & $x$ & $\mathrm{x}$ & $\mathrm{x}$ & $\mathrm{x}$ \\
\hline $\mathrm{Cr}$ & $\mathrm{x}$ & $\mathrm{x}$ & $\mathrm{x}$ & $\mathrm{X}$ \\
\hline $\mathrm{Cu}$ & $\mathrm{X}$ & $\mathrm{x}$ & $\mathrm{x}$ & $\mathrm{x}$ \\
\hline $\mathrm{Fe}$ & $x$ & $x$ & & \\
\hline $\mathrm{Hg}$ & $\mathrm{X}$ & $\mathrm{x}$ & $\mathrm{X}$ & $\mathrm{X}$ \\
\hline $\mathrm{K}$ & $\mathrm{x}$ & $x$ & & \\
\hline $\mathrm{Mg}$ & $X$ & $\mathrm{X}$ & & \\
\hline Mn & $\mathrm{x}$ & $\mathrm{x}$ & & \\
\hline $\mathrm{Na}$ & $x$ & $x$ & $\mathrm{x}$ & $\mathrm{X}$ \\
\hline $\mathrm{Ni}$ & $\mathrm{X}$ & $\mathrm{X}$ & $\mathrm{X}$ & $\mathrm{X}$ \\
\hline $\mathrm{Pb}$ & $\mathrm{X}$ & $\mathrm{x}$ & $\mathrm{x}$ & $\mathrm{x}$ \\
\hline $\mathrm{Se}$ & & & $\mathrm{X}$ & $\mathrm{X}$ \\
\hline$S i$ & $\mathrm{x}$ & & & \\
\hline $\mathrm{sn}$ & $\mathrm{x}$ & $\mathrm{x}$ & & \\
\hline Th & & $\mathrm{x}$ & & \\
\hline $\mathrm{Zn}$ & $\mathrm{x}$ & $\mathrm{X}$ & $\mathrm{X}$ & $X$ \\
\hline Alkalinity, Acidity & $x$ & & $\mathrm{x}$ & \\
\hline Chloride & $\mathrm{x}$ & & $\mathrm{x}$ & $\mathrm{x}$ \\
\hline Nitrogen $\left(\mathrm{NH}_{3}, \mathrm{NO}_{3}\right)$ & $\mathrm{x}$ & & $\mathrm{x}$ & $\mathrm{x}$ \\
\hline Phosphate & $\mathrm{X}$ & & & \\
\hline Total Hardness & & & $\mathrm{X}$ & \\
\hline $\mathrm{pH}$ & $x$ & & $\mathrm{X}$ & \\
\hline Conductivity & $\mathrm{x}$ & & $\mathrm{X}$ & \\
\hline
\end{tabular}


Table 1. (continued)

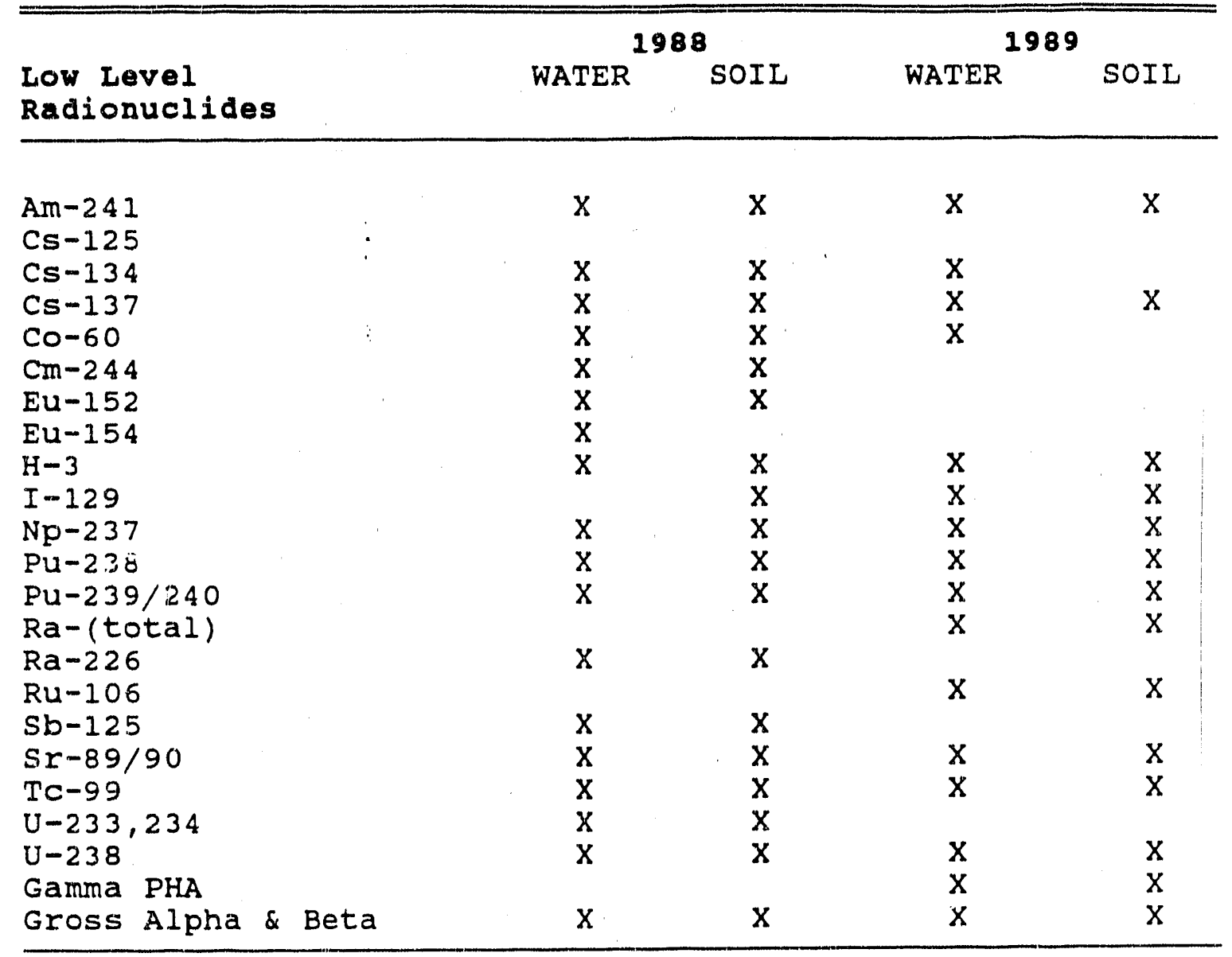


Table 2. Tritium concentrations and conductivities of Four Mile Creek Seepline Water (Sample Date: 3/89).

\begin{tabular}{|c|c|c|c|c|}
\hline Sample & \multicolumn{2}{|c|}{ SRS Coordinate } & $\begin{array}{l}\text { Tritium } \\
(\mathrm{pCi} / \mathrm{ml})\end{array}$ & $\begin{array}{c}\text { Conductivity } \\
(\mu \mathrm{mhos} / \mathrm{cm})\end{array}$ \\
\hline HSP-I & 70574 & 57141 & 1600 & 156 \\
\hline HSP-2 & 70642 & 56992 & 7100 & 256 \\
\hline HSP-3 & 70711 & 56879 & 14000 & 468 \\
\hline HSP- 4 & 70710 & 56819 & 11000 & 292 \\
\hline $\mathrm{HSP}-5$ & 70769 & 56819 & 9900 & 281 \\
\hline HSP- 6 & 70809 & 56868 & 12000 & 318 \\
\hline HSP -8 & 71005 & 56990 & 24000 & 556 \\
\hline HSP-9 & 70970 & 57141 & 12000 & 317 \\
\hline HSP-10 & 71044 & 57142 & 1700 & 171 \\
\hline HSP-11 & 71099 & 57046 & 960 & 80 \\
\hline$H S P-12$ & 71115 & 56960 & 4500 & 218 \\
\hline HSP -13 & 71056 & 56878 & 12000 & 592 \\
\hline HSP- 14 & 71038 & 56810 & 6800 & 393 \\
\hline HSP-15 & 71085 & 56791 & 1000 & 82 \\
\hline HSP-16 & 71150 & 56786 & 2500 & 216 \\
\hline HSP-18 & 71276 & 56662 & 2700 & 149 \\
\hline HSP-19 & 71253 & 56539 & 3400 & 148 \\
\hline HSP -20 & 71142 & 56489 & 6500 & 183 \\
\hline HSP-2 I & 71028 & 56520 & 5800 & 189 \\
\hline HSP -22 & 70989 & 56483 & 5100 & 161 \\
\hline HSP -23 & 70921 & 56500 & 6600 & 184 \\
\hline HSP-24 & 70910 & 56347 & 3100 & 140 \\
\hline HSP -25 & 70960 & 56297 & 3300 & 135 \\
\hline HSP -26 & 71016 & 562.36 & 6600 & 205 \\
\hline HSP -27 & 71121 & 56208 & 4500 & 202 \\
\hline HSP -28 & 71194 & 56192 & 5700 & 244 \\
\hline HSP-29 & 71278 & 56257 & 9200 & 257 \\
\hline HSP -30 & 71397 & 56302 & 3400 & 121 \\
\hline$H S P-31$ & 71490 & 56342 & 4100 & 214 \\
\hline HSP -32 & 71580 & 56281 & 3100 & 215 \\
\hline HSP -33 & 71656 & 56262 & 3800 & .176 \\
\hline HSP-34 & 71698 & 56193 & 5600 & 331 \\
\hline HSP -35 & 71768 & 56185 & 2200 & 121 \\
\hline HSP-36 & 71813 & 56148 & 2800 & 210 \\
\hline HSP -37 & 71778 & 56037 & 7600 & 231 \\
\hline HSP -38 & 71788 & 55969 & 6500 & 227 \\
\hline HSP -39 & 71838 & 55903 & 6700 & 242 \\
\hline HSP-40 & 71844 & 55773 & 7200 & 256 \\
\hline HSP-4 1 & 71768 & 55736 & 5700 & 224 \\
\hline HSP -42 & 71691 & 55749 & 8500 & 310 \\
\hline HSP -43 & 71644 & 55722 & 10000 & 413 \\
\hline $\mathrm{HSP}-44$ & 71659 & 55818 & 14000 & 333 \\
\hline$H S P-45$ & 71653 & 55917 & 5500 & 255 \\
\hline HSP -46 & 71621 & 56052 & 11000 & 318 \\
\hline HSP -47 & 71534 & 56077 & 19000 & 426 \\
\hline HSP -48 & 71455 & 56059 & 21000 & 569 \\
\hline HSP -49 & 71389 & 55987 & 11000 & 551 \\
\hline $\mathrm{HSP}-50$ & 71338 & 55903 & 18000 & 537 \\
\hline
\end{tabular}


Table 2. Continued (Sample Date: 3/89)

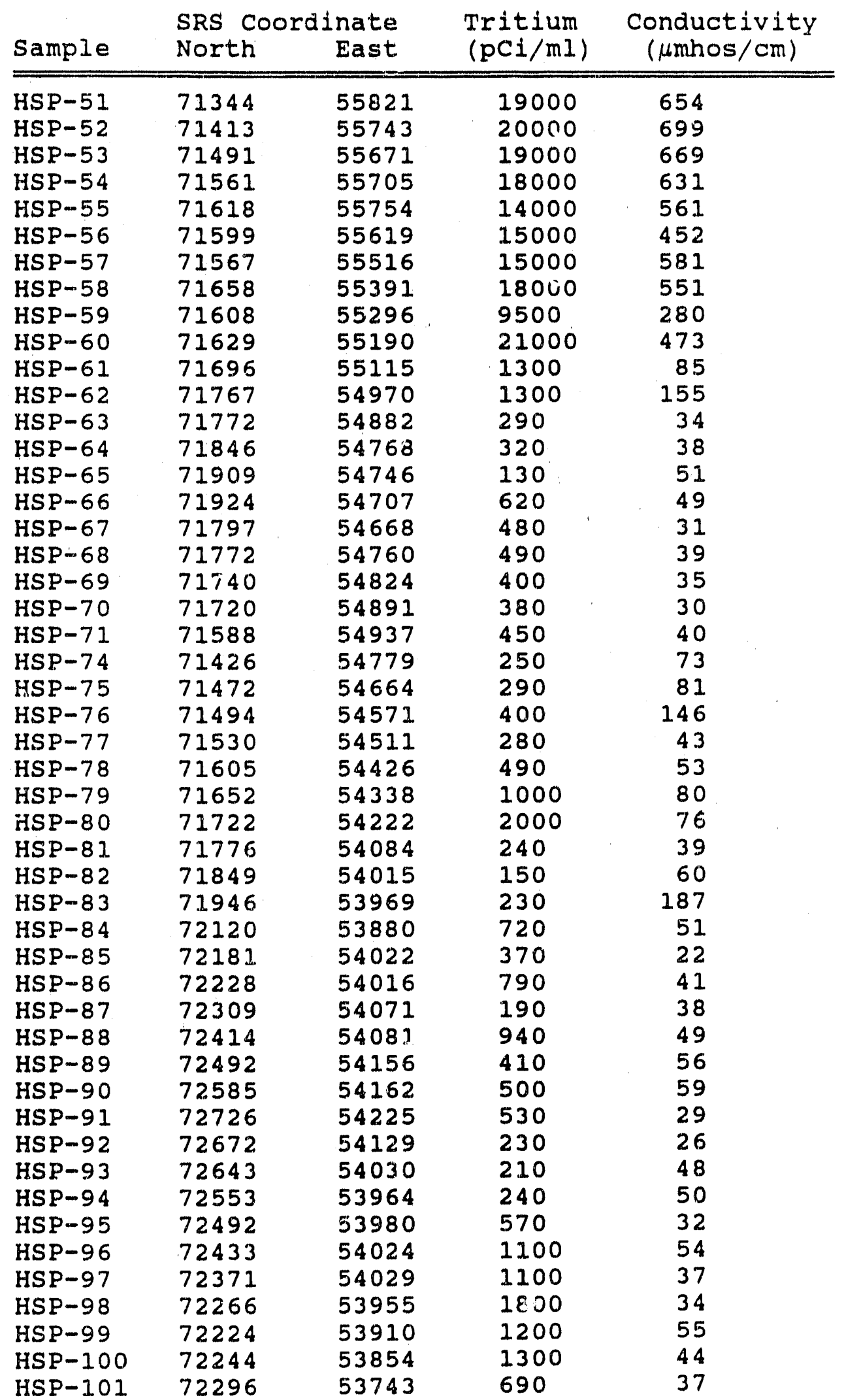


Table 2. Continued (Sample Date: 3/89)

\begin{tabular}{|c|c|c|c|c|}
\hline Sample & \multicolumn{2}{|c|}{ SRS Coordinate } & $\begin{array}{l}\text { Tritium } \\
(\mathrm{pci} / \mathrm{ml})\end{array}$ & 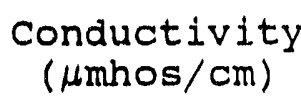 \\
\hline HSP-102 & 72347 & 53673 & 700 & 40 \\
\hline HSP-103 & 72448 & 53665 & 510 & 43 \\
\hline HSP-104 & 72529 & 53539 & 660 & 38. \\
\hline HSP-105 & 72632 & 53427 & 740 & 44 \\
\hline HSP-106 & 72720 & 53376 & 640 & 60 \\
\hline HSP -107 & 72806 & 53410 & 480 & 47 \\
\hline HSP-108 & 72887 & 53419 & 600 & 58 \\
\hline HSP-109 & 72933 & 53294 & 680 & 73 \\
\hline HSP-201 & 70409 & 57235 & 470 & 74 \\
\hline HSP -202 & 70473 & 57346 & 560 & 56 \\
\hline HSP -203 & 70448 & 57445 & 940 & 110 \\
\hline HSP-205 & 70282 & 57522 & 12 & 139 \\
\hline HSP -206 & 70143 & 57525 & 19 & 62 \\
\hline HSP -207 & 70094 & 57506 & 17 & 135 \\
\hline HSP -208 & 70029 & 57451 & 19 & 84 \\
\hline HSP-209 & 69906 & 57444 & 20 & 72 \\
\hline HSP -210 & 69859 & 57537 & 15 & 55 \\
\hline$H S P-211$ & 69876 & 57624 & 18 & 43 \\
\hline HSP -212 & 69858 & 57690 & 19 & 27 \\
\hline $\mathrm{HSP}-213$ & 69796 & 57740 & 15 & 57 \\
\hline HSP-2 14 & 69690 & 57759 & 18 & 37 \\
\hline HSP-2 15 & 69586 & 57790 & 16 & 58 \\
\hline HSP -216 & 69500 & 57865 & 10 & 56 \\
\hline HSP -217 & 69468 & 57939 & 11 & 50 \\
\hline HSP $-101 \mathrm{~N}$ & 70622 & 56346 & 22 & 31 \\
\hline $\mathrm{HSP}-102 \mathrm{~N}$ & 70570 & 56437 & 22 & 36 \\
\hline $\mathrm{HSP}-103 \mathrm{~N}$ & 70528 & 56524 & 18 & 26 \\
\hline HSP $-104 \mathrm{~N}$ & 70468 & 56620 & 150 & 56 \\
\hline HSP-105N & 70431 & 56698 & 71 & 70 \\
\hline HSP-106N & 70429 & 56761 & 18 & 29 \\
\hline $\mathrm{HSP}-107 \mathrm{~N}$ & 70419 & 56838 & 19 & 47 \\
\hline HSP $-108 \mathrm{~N}$ & 70405 & 56915 & 18 & 57 \\
\hline HSP-109N & 70340 & 56974 & 22 & 39 \\
\hline HSP-110N & 70304 & 57138 & 19 & 44 \\
\hline HSP-111N & 70269 & 57192 & 21 & 44 \\
\hline HSP-112N & 70208 & 57295 & 12 & 60 \\
\hline $\mathrm{HSP}-113 \mathrm{~N}$ & 70122 & 57308 & 14 & 46 \\
\hline $\mathrm{HSP}-114 \mathrm{~N}$ & 70066 & 57280 & 18 & 47 \\
\hline HSP-115N & 70000 & 57257 & 15 & 32 \\
\hline $\mathrm{HSP}-116 \mathrm{~N}$ & 69922 & 57274 & 21 & 32 \\
\hline HSP-101S & 70403 & 55016 & 18 & 33 \\
\hline HSP-102S & 70525 & 55003 & 24 & 31 \\
\hline HSP-103S & 70621 & 54989 & 17 & 35 \\
\hline HSP $-104 S$ & 70705 & 54946 & 21 & 28 \\
\hline HSP-105S & 70768 & 54873 & 20 & 26 \\
\hline HSP-106S & 70786 & 54788 & 23 & 30 \\
\hline HSP-107S & 70854 & 54710 & 23 & 46 \\
\hline HSP-108S & 70885 & 54631 & 21 & 32 \\
\hline HSP-109S & 70950 & 54550 & 20 & 29 \\
\hline
\end{tabular}


Table 2. Continued (Sample Date: 3/89)

\begin{tabular}{|c|c|c|c|c|}
\hline Sample & $\begin{array}{l}\text { SRS C } \\
\text { North }\end{array}$ & $\begin{array}{l}\text { inate } \\
\text { East }\end{array}$ & $\begin{array}{l}\text { Tritium } \\
(\mathrm{pCi} / \mathrm{ml})\end{array}$ & $\begin{array}{c}\text { Conductivity } \\
(\mu \operatorname{mhos} / \mathrm{cin})\end{array}$ \\
\hline HSP-110S & 71038 & 54509 & 23 & 19 \\
\hline HSP-111S & 71112 & 54464 & 16 & 26 \\
\hline $\mathrm{HSP}-112 \mathrm{~S}$ & 71201 & 54486 & 29 & 42 \\
\hline HSP-113S & 71256 & 54443 & 22 & 36 \\
\hline$T-1$ & 71248 & 55795 & 21000 & \\
\hline $\mathrm{T}-2$ & 71150 & 55769 & 1600 & \\
\hline$T-3$ & 71051 & 55742 & 8200 & \\
\hline$T-4$ & 70957 & 55717 & 10000 & \\
\hline$T-5$ & 70861 & 55691 & 140 & \\
\hline$T-6$ & 70764 & 55665 & 31 & \\
\hline$T-7$ & 70668 & 55639 & 26 & \\
\hline FSP-1 & 72776 & 49011 & 1100 & 72 \\
\hline FSP -2 & 72796 & 49133 & 520 & 94 \\
\hline FSP-3 & 72830 & 49222 & 70 & 42 \\
\hline FSP -4 & 72877 & 49355 & 1600 & 122 \\
\hline FSP- 5 & 72965 & 49414 & 2400 & 425 \\
\hline FSP -6 & 73009 & 49460 & 1700 & 236 \\
\hline FSP-7 & 73008 & 49540 & 3400 & 681 \\
\hline FSP -8 & 73080 & 49562 & 3000 & 1175 \\
\hline FSP-9 & 73221 & 49626 & 2900 & 589 \\
\hline $15 P-10$ & 73375 & 49600 & 850 & 167 \\
\hline FSP-11 & 73475 & 49707 & 4100 & 417 \\
\hline FSP-12 & 73602 & 49644 & 260 & 30 \\
\hline FSP-13 & 73674 & 49656 & 69 & 32 \\
\hline FSP-14 & 73685 & 49802 & 14000 & 666 \\
\hline FSP-15 & 73576 & 49863 & 1200 & 52 \\
\hline FSP-16 & 73521 & 49871 & 8600 & 714 \\
\hline FSP -17 & 73439 & 49907 & 8600 & 794 \\
\hline FSP-18 & 73308 & 49791 & 5200 & 1043 \\
\hline FSP- 19 & 73177 & 49778 & 4900 & 1424 \\
\hline FSP-20 & 73060 & 49741 & 6600 & 1193 \\
\hline FSP -21 & 73026 & 49608 & 7600 & 1029 \\
\hline FSP-22 & 72953 & 49585 & 3600 & 1015 \\
\hline FSP-23 & 72862 & 49554 & 3100 & 851 \\
\hline FSP-24 & 72803 & 49665 & 2700 & 1325 \\
\hline FSP-25 & 72821 & 49764 & 3000 & 790 \\
\hline FSP-26 & 72932 & 49801 & 4400 & 1095 \\
\hline FSP-27 & 73060 & 49856 & 3400 & 780 \\
\hline FSP-28 & 73082 & 49980 & 4000 & 1203 \\
\hline FSP-29 & 73143 & 50134 & 4600 & 534 \\
\hline FSP-30 & 73216 & 50164 & 5200 & 490 \\
\hline FSP-31 & 73330 & 50132 & 970 & 44 \\
\hline FSP -32 & 73367 & 50258 & 3600 & 174 \\
\hline FSP-33 & 73306 & 50302 & 10000 & 540 \\
\hline FSP-34 & 73156 & 50261 & 14000 & 810 \\
\hline FSP-35 & 73065 & 50297 & 11000 & 1100 \\
\hline FSP -36 & 72940 & 50292 & 8900 & 1200 \\
\hline FSP-37 & 72960 & 50368 & 8800 & 1660 \\
\hline FSP -38 & 73019 & 50514 & 7100 & 800 \\
\hline
\end{tabular}


Table 2. Continued (Sample Date: 3/89)

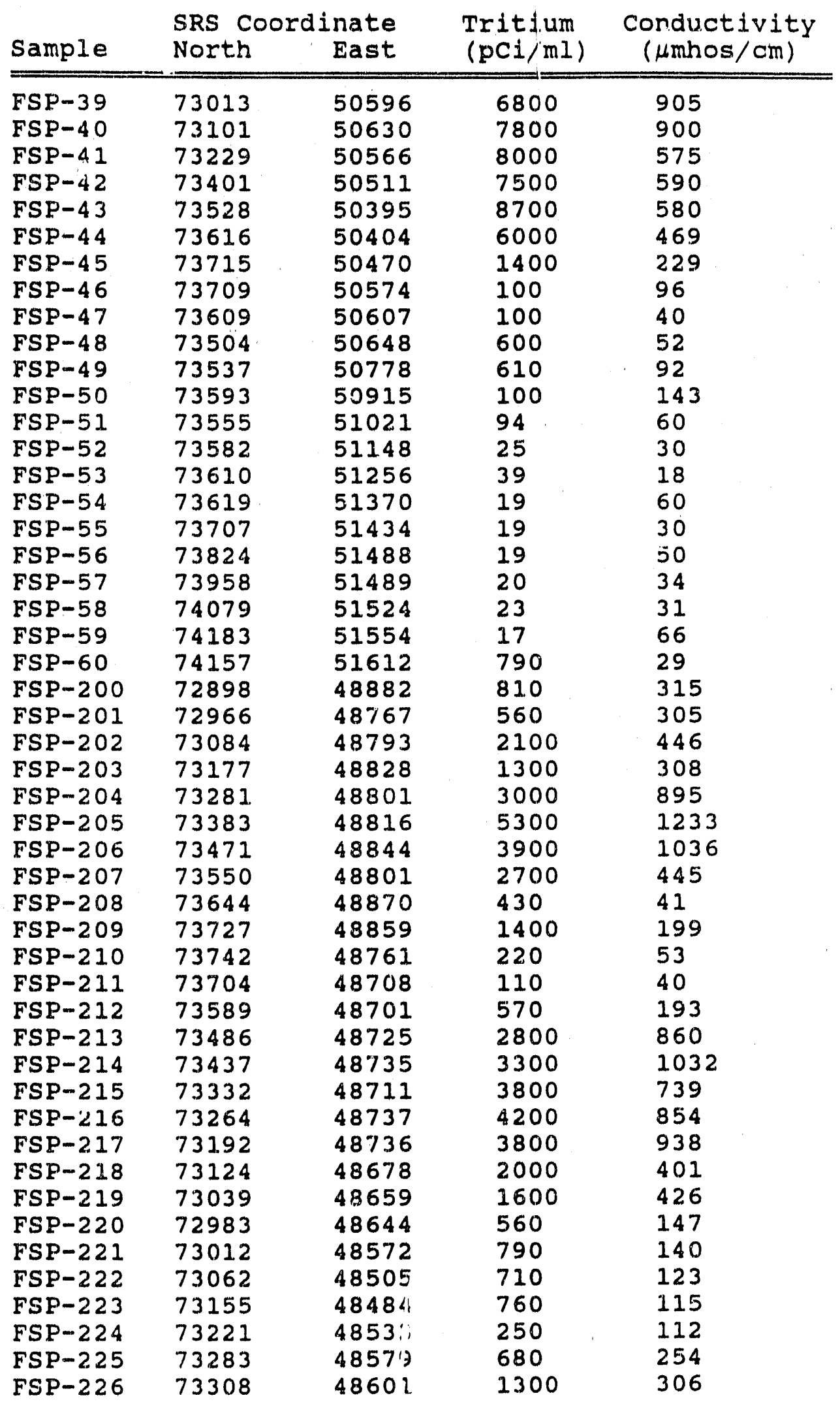


Table 2. Continued (Sample Date: 3/89)

\begin{tabular}{|c|c|c|c|c|}
\hline Sample & \multicolumn{2}{|c|}{ SRS Coordinate } & $\begin{array}{l}\text { Tritium } \\
(\mathrm{pCi} / \mathrm{ml})\end{array}$ & $\begin{array}{c}\text { Conductivity } \\
(\mu \text { mhos } / \mathrm{cm})\end{array}$ \\
\hline FSP-2 27 & 73324 & 48501 & 1100 & 204 \\
\hline FSP-228 & 73305 & 48428 & 1500 & 259 \\
\hline FSP-2,29 & 73305 & 48331 & 350 & 54 \\
\hline FSP-230 & 73246 & 48298 & 430 & 95 \\
\hline FSP-231 & 73181 & 48373 & 250 & 70 \\
\hline FSP -232 & 73169 & 48456 & 520 & 96 \\
\hline FSP -233 & 73118 & 48468 & 650 & 103 \\
\hline FSP-234 & 73034 & 48482 & 670 & 66 \\
\hline FSP-235 & 72979 & 48587 & 580 & 84 \\
\hline FSP-236 & 72943 & 48599 & 530 & 112 \\
\hline FSP- 237 & 72915 & 48536 & 310 & 67 \\
\hline FSP -238 & 72939 & 48453 & 680 & 79 \\
\hline FSP-239 & 72937 & 48367 & 250 & 37 \\
\hline FSP -240 & 72878 & 48287 & 57 & 42 \\
\hline FSP -241 & 72853 & 48190 & 560 & 36 \\
\hline FSP-242 & .72780 & 48085 & 180 & 53 \\
\hline FSP-243 & 72786 & 47980 & 390 & 48 \\
\hline FSP-244 & 72806 & 47918 & 280 & 50 \\
\hline FSP -245 & 72794 & 47833 & 980 & 116 \\
\hline FSP-246 & 72856 & 47842 & 780 & 90 \\
\hline FSP-247 & 72946 & 47901 & 590 & 102 \\
\hline FSP -248 & 7.3030 & 47918 & 960 & 120 \\
\hline FSP -249 & 73107 & 47905 & 580 & 84 \\
\hline FSP -250 & 731.56 & 47867 & 200 & 42 \\
\hline FSP -251 & 73280 & 47881 & 460 & 59 \\
\hline FSP -252 & 73341 & 47913 & 570 & 66 \\
\hline FSP -253 & 73403 & 47938 & 210 & 31 \\
\hline FSP-254 & 73446 & 47896 & 90 & 41 \\
\hline FSP-255 & 73471 & 47821 & 370 & 52 \\
\hline FSP -256 & 73435 & 47770 & 400 & 56 \\
\hline FSP-257 & 73385 & 47735 & 270 & 46 \\
\hline FSP -258 & 73307 & 47777 & 510 & 49 \\
\hline FSP -259 & 73225 & 47779 & 150 & 78 \\
\hline FSP-260 & 73148 & 47794 & 360 & 61 \\
\hline FSP-261 & 73071 & 47800 & 730 & 91 \\
\hline FSP-262 & 72988 & 47811 & 630 & 64 \\
\hline FSP -263 & 72918 & 47797 & 220 & 59 \\
\hline FSP-264 & 72873 & 47758 & 100 & 57 \\
\hline FSP-265 & 72844 & 47697 & 110 & 62 \\
\hline FSP-266 & 72805 & 47594 & 92 & 44 \\
\hline FSP-267 & 72724 & 47578 & 94 & 85 \\
\hline FSP -268 & 72703 & 47545 & 270 & 35 \\
\hline FSP -269 & 72777 & 47509 & 32 & 28 \\
\hline FSP -270 & 72805 & $\$ 7411$ & 40 & 50 \\
\hline FSP -271 & 72779 & 47393 & 62 & 29 \\
\hline FSP -272 & 72773 & 47362 & 45 & 39 \\
\hline FSP -273 & 72842 & 47349 & 32 & 54 \\
\hline FSP-274 & 72850 & 47278 & 49 & 39 \\
\hline FSP -275 & 72847 & 47193 & 39 & 69 \\
\hline
\end{tabular}


Table 2. Continued (Sample Date: 3/89)

\begin{tabular}{|c|c|c|c|c|}
\hline Sample & $\begin{array}{l}\text { SRS Co } \\
\text { North }\end{array}$ & $\begin{array}{l}\text { nate } \\
\text { East }\end{array}$ & $\begin{array}{l}\text { Tritium } \\
(\mathrm{pCi} / \mathrm{ml})\end{array}$ & $\begin{array}{c}\text { Conductivity } \\
(\mu \text { mhos/cm) }\end{array}$ \\
\hline $\begin{array}{l}\text { FSP-276 } \\
\text { FSP-277 } \\
\text { FSP-2 } 28 \\
\text { FSP-279 } \\
\text { FSP-280 } \\
\text { FSP-281 } \\
\text { FSP-282 } \\
\text { FSP-283 } \\
\text { FSP-284 } \\
\text { FSP-285 } \\
\text { FSP-286 } \\
\text { FSP-287 } \\
\text { FSP-288 } \\
\text { FSP-289 } \\
\text { FSP-290 } \\
\text { FSP-291 } \\
\text { FSP-292 } \\
\text { FSP-293 } \\
\text { FSP-294 } \\
\text { FSP-295 } \\
\text { FSP-296 } \\
\text { FSP-297 } \\
\text { FSP-298 } \\
\text { FSP-299 } \\
\text { FSP-300 } \\
\text { FSP-301 } \\
\text { FSP-302 } \\
\text { FSP-303 } \\
\text { FSP-304 } \\
\text { FSP-305 } \\
\text { FSP-306 } \\
\text { FSP-307 } \\
\text { FSP-308 } \\
\text { FSP-309 }\end{array}$ & $\begin{array}{l}72823 \\
72788 \\
72853 \\
72833 \\
72837 \\
72824 \\
72869 \\
72887 \\
72870 \\
72884 \\
72961 \\
73043 \\
73094 \\
73045 \\
73160 \\
73235 \\
73209 \\
73136 \\
73083 \\
73026 \\
72924 \\
72904 \\
72886 \\
72864 \\
72848 \\
72842 \\
72804 \\
72798 \\
72795 \\
72773 \\
72769 \\
72831 \\
72899 \\
72971\end{array}$ & $\begin{array}{l}47118 \\
47086 \\
47058 \\
46982 \\
46853 \\
46785 \\
46720 \\
46672 \\
46608 \\
46560 \\
46603 \\
46676 \\
46724 \\
46791 \\
46865 \\
46717 \\
46681 \\
46677 \\
46635 \\
46573 \\
46540 \\
46541 \\
46453 \\
46402 \\
46364 \\
46277 \\
46209 \\
46145 \\
46070 \\
45993 \\
45919 \\
45937 \\
45968 \\
45947\end{array}$ & $\begin{array}{l}30 \\
28 \\
24 \\
27 \\
24 \\
31 \\
22 \\
33 \\
33 \\
99 \\
45 \\
72 \\
59 \\
25 \\
35 \\
19 \\
20 \\
18 \\
19 \\
17 \\
10 \\
15 \\
17 \\
20 \\
17 \\
16 \\
16 \\
58 \\
16 \\
17 \\
16 \\
18 \\
18 \\
13\end{array}$ & $\begin{array}{l}56 \\
49 \\
42 \\
47 \\
47 \\
55 \\
37 \\
95 \\
57 \\
37 \\
24 \\
22 \\
83 \\
53 \\
49 \\
24 \\
24 \\
36 \\
49 \\
56 \\
18 \\
44 \\
46 \\
44 \\
37 \\
34 \\
29 \\
94 \\
52 \\
41 \\
23 \\
29 \\
33 \\
36\end{array}$ \\
\hline
\end{tabular}


Table 2. Continued (Sample Date 1/90)

\begin{tabular}{|c|c|c|c|c|}
\hline Sample & \multicolumn{2}{|c|}{ SRS Coordinate } & $\begin{array}{l}\text { Tritium } \\
\text { (pci/ml) }\end{array}$ & $\begin{array}{c}\text { Conductivity } \\
\text { ( } \mu \text { mhos/cm })\end{array}$ \\
\hline FSP-101E & 72496 & 49222 & 50 & 82 \\
\hline FSP-102E & 72483 & 49339 & 65 & 82 \\
\hline FSP-103E & 72507 & 49379 & 60 & 36 \\
\hline FSP-104E & 72545 & 49399 & 32 & 36 \\
\hline FSP-105E & 72598 & 49470 & 58 & 27 \\
\hline F'SP-106E & 72648 & 49527 & 43 & 78 \\
\hline FSP $-107 \mathrm{E}$ & 72633 & 49628 & 18 & 22 \\
\hline FSP $-108 E$ & 72605 & 49754 & 63 & 13 \\
\hline FSP-109E & 72630 & 49836 & 68 & 25 \\
\hline FSP-110E & 72647 & 49910 & 45 & 20 \\
\hline FSP-IIIE & 72667 & 49989 & 73 & 25 \\
\hline FSP-112E & 72683 & 50060 & 78 & 22 \\
\hline FSP-113E & 72675 & 50142 & 38 & 17 \\
\hline FSP-114E & 72706 & 50221 & 28 & 60 \\
\hline FSP-115E & 72710 & 50301 & 82 & 14 \\
\hline FSP-I16E & 72686 & 50359 & 44 & 20 \\
\hline FSP-117E & 72685 & 50421 & 39 & 19 \\
\hline FSP $-101 \mathrm{~W}$ & 72505 & 48015 & 80 & 228 \\
\hline FSP-102W & 72500 & 48107 & 110 & 12.3 \\
\hline FSP-103W & 72535 & 48178 & 53 & 39 \\
\hline FSP-104W & 72585 & 48261 & 48 & 55 \\
\hline FSP $-105 \mathrm{~W}$ & 72606 & 48339 & 55 & 62 \\
\hline FSP-106W & 72648 & 48412 & 83 & 61 \\
\hline FSP $-107 \mathrm{~W}$ & 72626 & 48472 & 71 & 43 \\
\hline FSP-108W & 7261.3 & 48544 & 32 & 60 \\
\hline FSP-109W & 72635 & 48635 & 28 & 53 \\
\hline FSP $-110 \mathrm{~W}$ & 72638 & 48722 & 34 & 79 \\
\hline FSP-61 & 74097 & 51686 & 18 & 32 \\
\hline FSP- 62 & 74027 & 51678 & 10 & 21 \\
\hline FSP -63 & 73964 & 51677 & 10 & 19 \\
\hline FSP-64 & 73908 & 51648 & $<2.2$ & 26 \\
\hline$F S P-65$ & 73828 & 51591 & 29 & 34 \\
\hline FSP-66 & 73752 & 51529 & NA & $\mathrm{NA}$ \\
\hline FSP -67 & 73681 & 51501 & 13 & 27 \\
\hline FSP-68 & 73627 & 51528 & $<2.2$ & 25 \\
\hline FSP-69 & 73603 & 51640 & 33 & 42 \\
\hline FSP-70 & 73625 & 51721 & 31 & 42 \\
\hline FSP-71 & 73674 & 51818 & NA & \\
\hline FSP-72 & 73699 & 51919 & 28 & 20 \\
\hline FSP -73 & 73674 & 51992 & 22 & 31 \\
\hline FSP -74 & 73636 & 52086 & 14 & 43 \\
\hline FSP -75 & 73607 & 52153 & 22 & 24 \\
\hline FSP -76 & 73666 & 52198 & 30 & 26 \\
\hline FSP -77 & 73631 & 52256 & 30 & 26 \\
\hline FSP-78 & 73514 & 52189 & 32 & 17 \\
\hline FSP-79 & 73446 & 52127 & 25 & 17 \\
\hline FSP -80 & 73336 & 52124 & 85 & 55 \\
\hline FSP-81 & 73281 & 52197 & 19 & 41 \\
\hline FSP -82 & 73324 & 52278 & 19 & 30 \\
\hline
\end{tabular}


Table 2. Continued (Sample Date: 1/90)

\begin{tabular}{lllll} 
Sample & $\begin{array}{c}\text { SRS Coordinate } \\
\text { North }\end{array}$ & $\begin{array}{c}\text { Tritium } \\
(\text { pCi/m })\end{array}$ & $\begin{array}{c}\text { Conductivity } \\
(\mu \text { mhos/cm) }\end{array}$ \\
\hline FSP-83 & 73383 & 52360 & 19 & 33 \\
FSP-84 & 73379 & 52424 & 180 & 39 \\
FSP-85 & 73354 & 52486 & 5.9 & 43 \\
FSP-86 & 73299 & 52536 & 49 & 33 \\
FSP-87 & 73239 & 52590 & 19 & 43 \\
FSP-88 & 73204 & 52666 & 35 & 19 \\
FSP-89 & 73220 & 52728 & 22 & 31 \\
FSP-90 & 73214 & 52788 & 31 & 28 \\
FSP-91 & 73152 & 52829 & 220 & 17 \\
FSP-92 & 73109 & 52898 & 140 & 18 \\
FSP-93 & 73026 & 52971 & 37 & 22 \\
HSP-94 & 72553 & 53963 & 750 & 28 \\
HSP-95 & 72492 & 53980 & 750 & 26 \\
HSP-96 & 72433 & 57023 & 1600 & 45 \\
HSP-97 & 72371 & 54029 & 1500 & 51 \\
HSP-98 & 72265 & 53954 & 1500 & 43 \\
HSP-99 & 72524 & 53909 & 1200 & 28 \\
HSP-100 & 72243 & 53854 & 1800 & 64 \\
HSP-101 & 72296 & 53742 & 950 & 28 \\
HSP-102 & 72347 & 53673 & 610 & 78 \\
HSP-103 & 72448 & 53664 & 400 & 58 \\
HSP-104 & 72529 & 53539 & 810 & 52 \\
HSP -105 & 72631 & 53427 & 810 & 103 \\
HSP-106 & 72720 & 53375 & 740 & 58 \\
HSP-107 & 72806 & 53409 & 460 & 124 \\
HSP-108 & 72887 & 53418 & 910 & 61 \\
HSP-109 & 72932 & 53293 & 230 & 75 \\
HSP & & & &
\end{tabular}




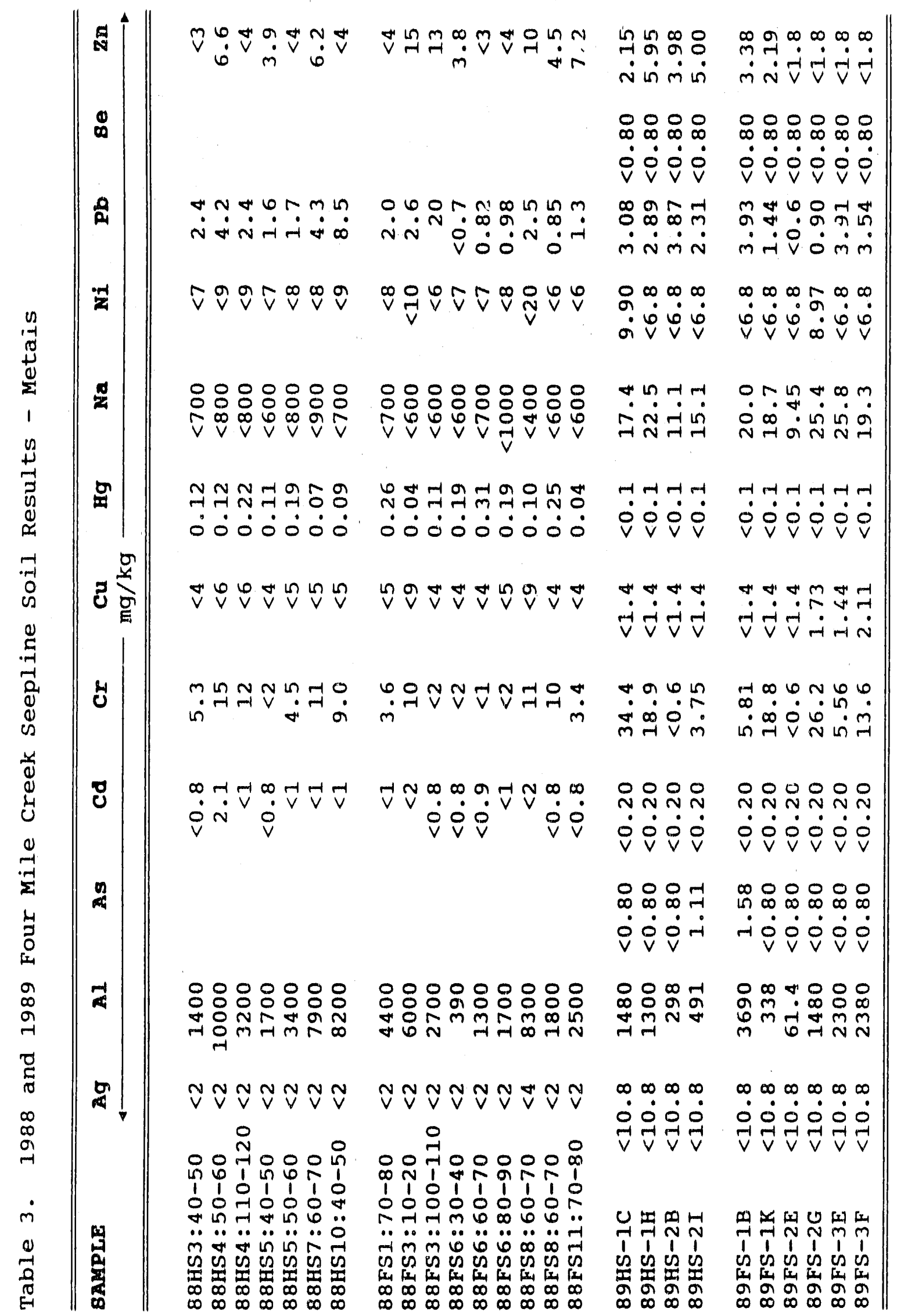




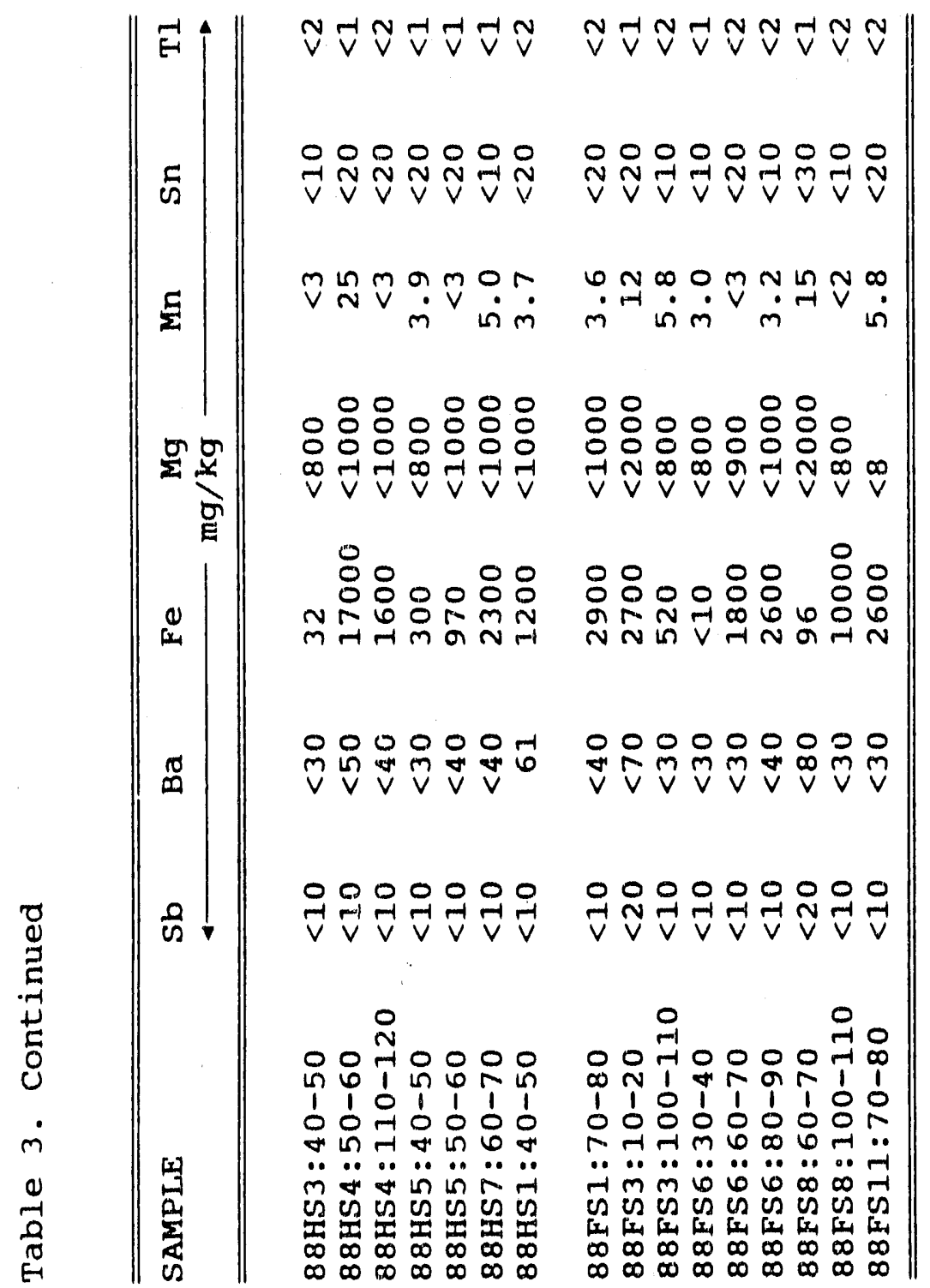




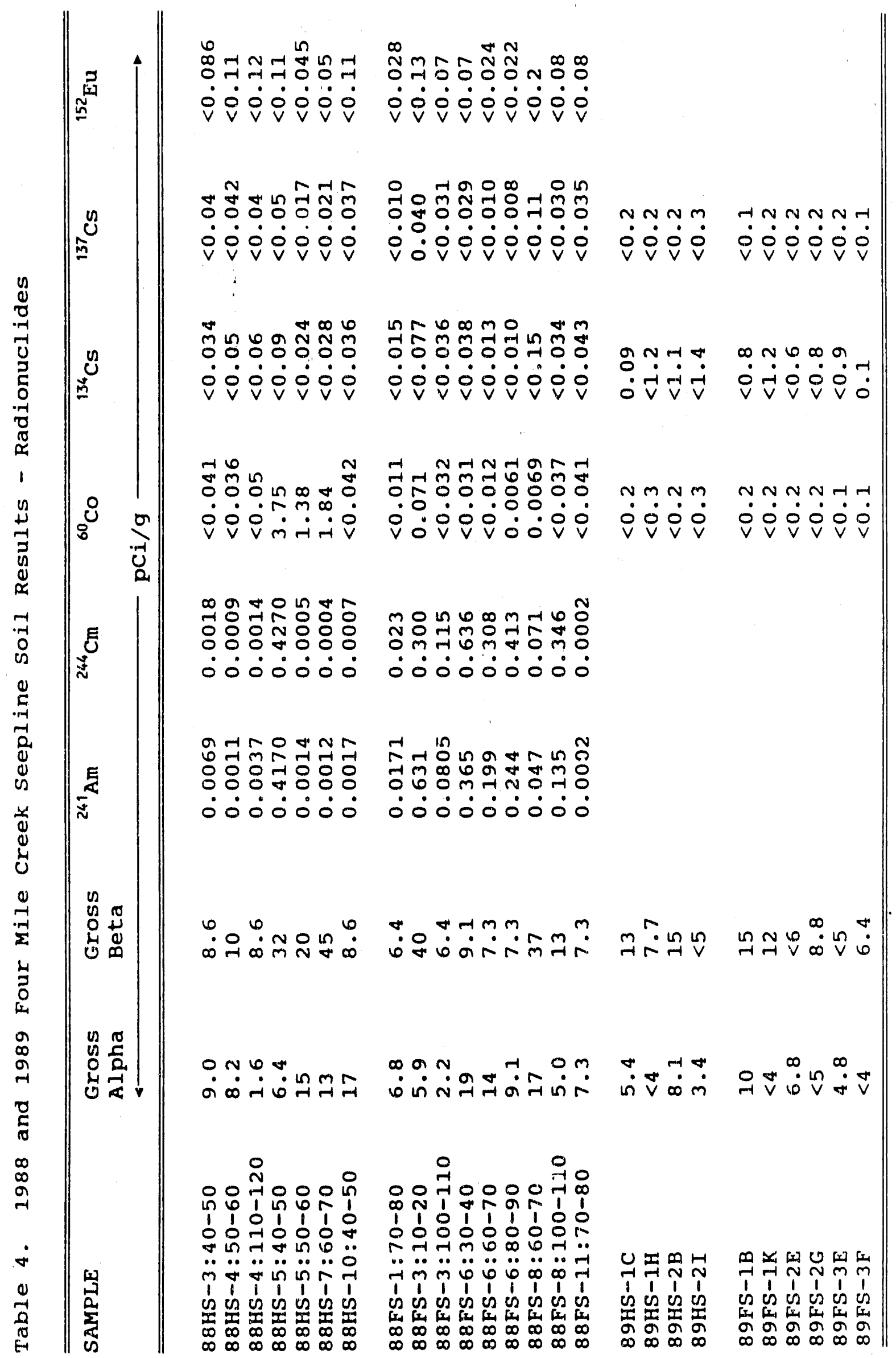




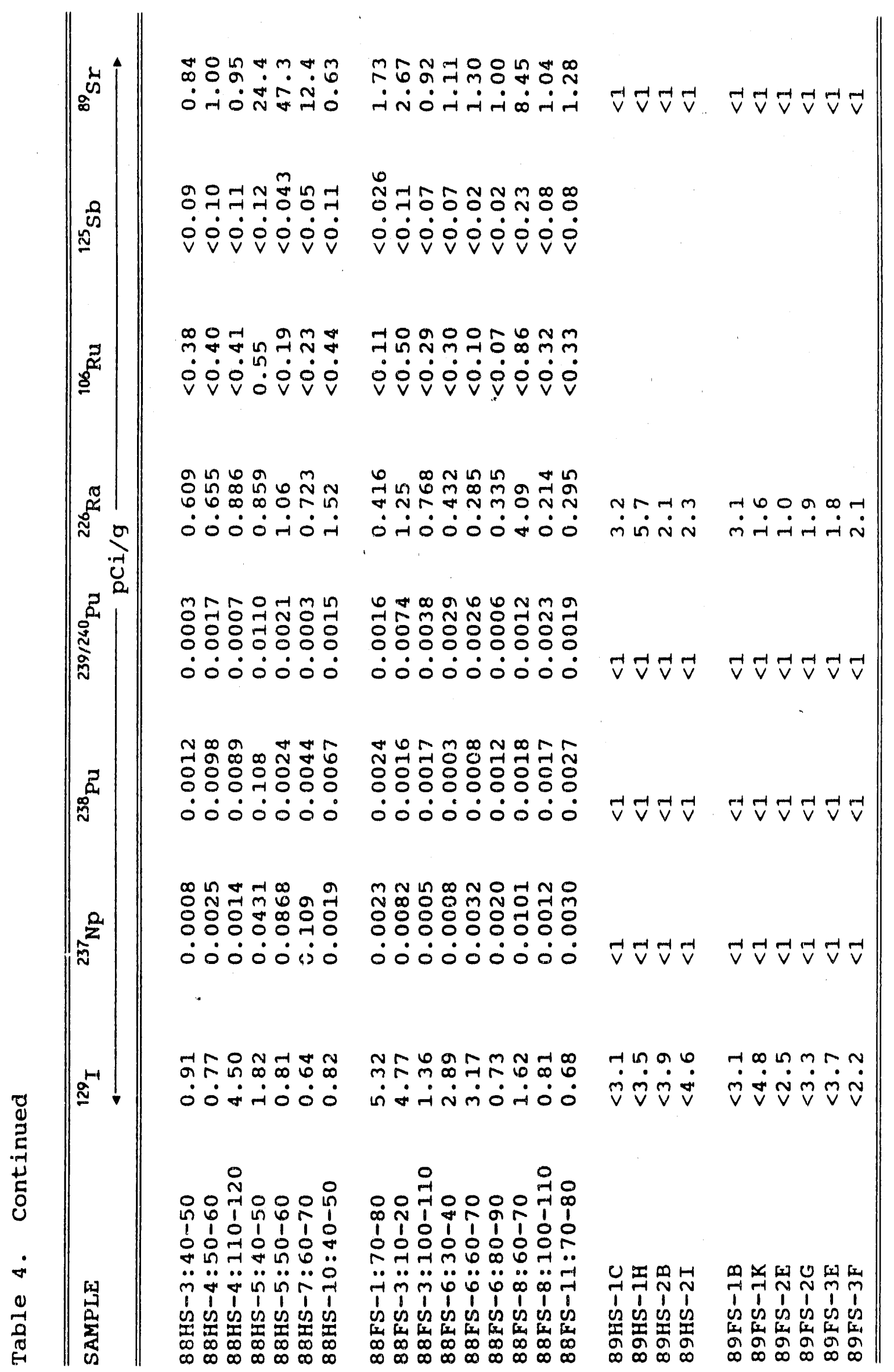




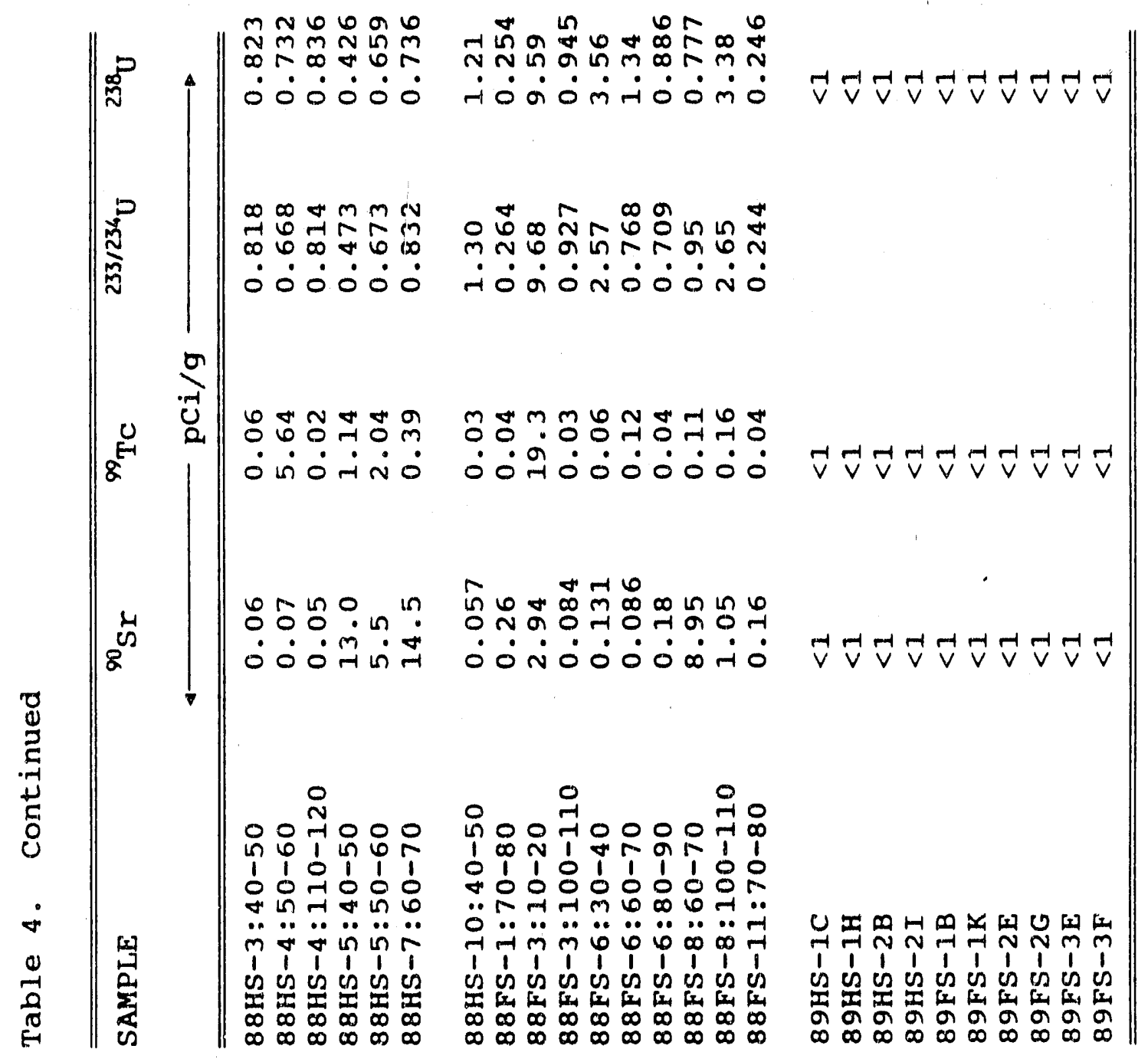




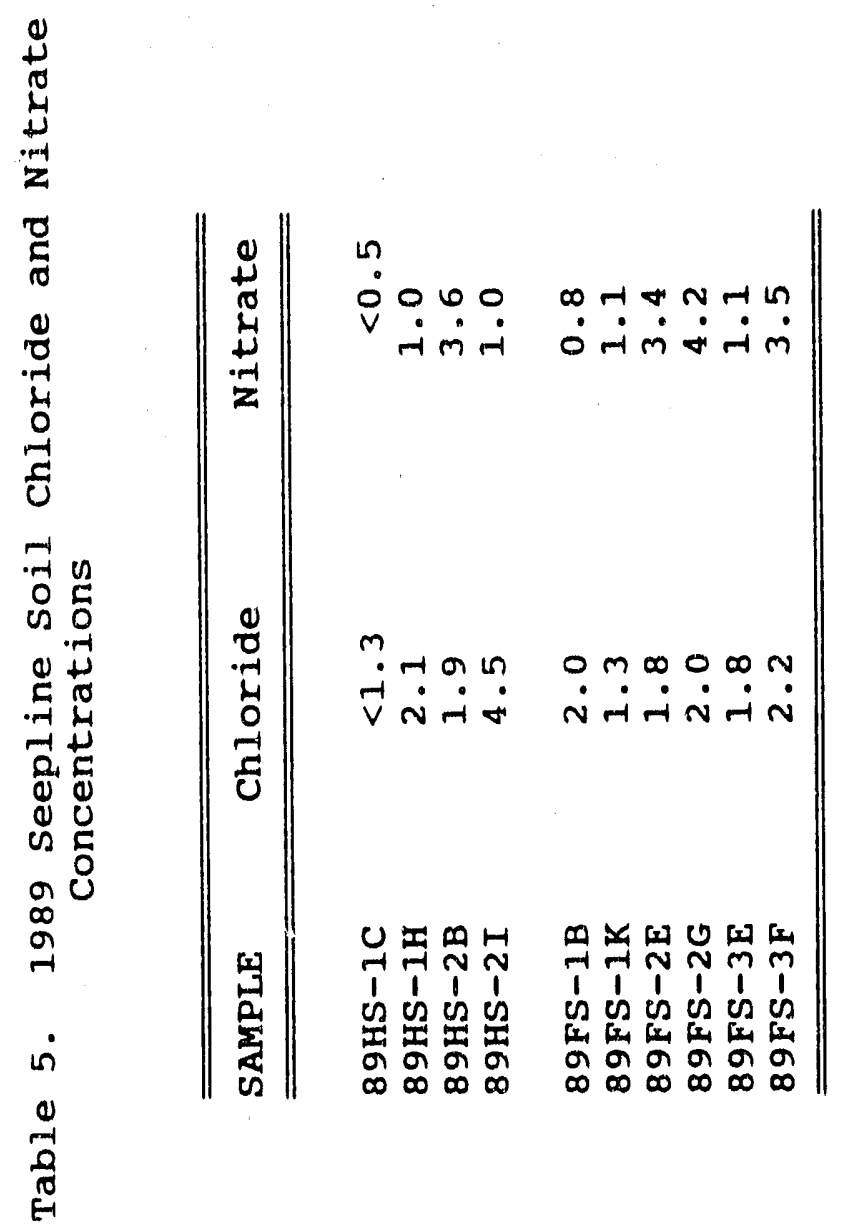




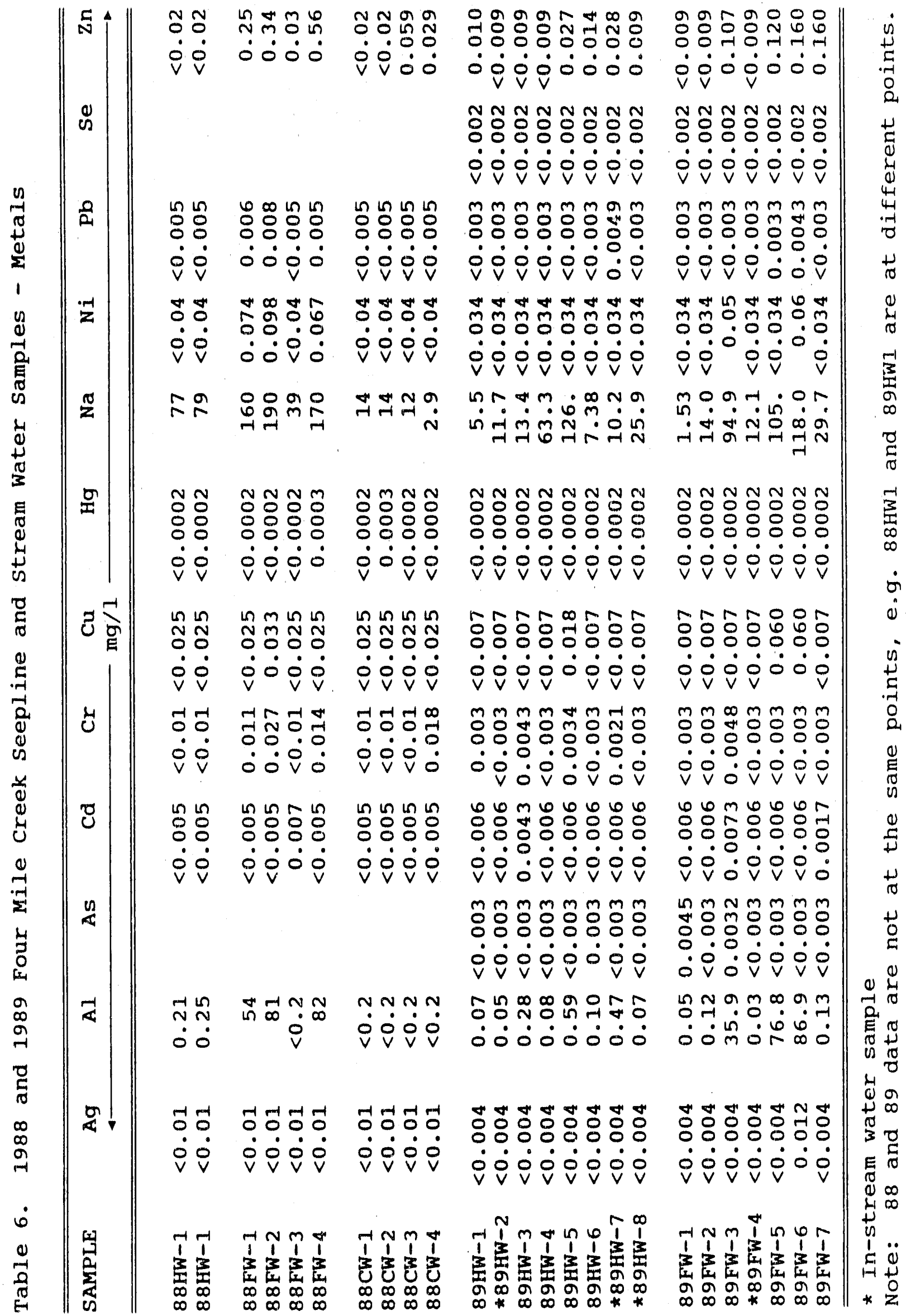




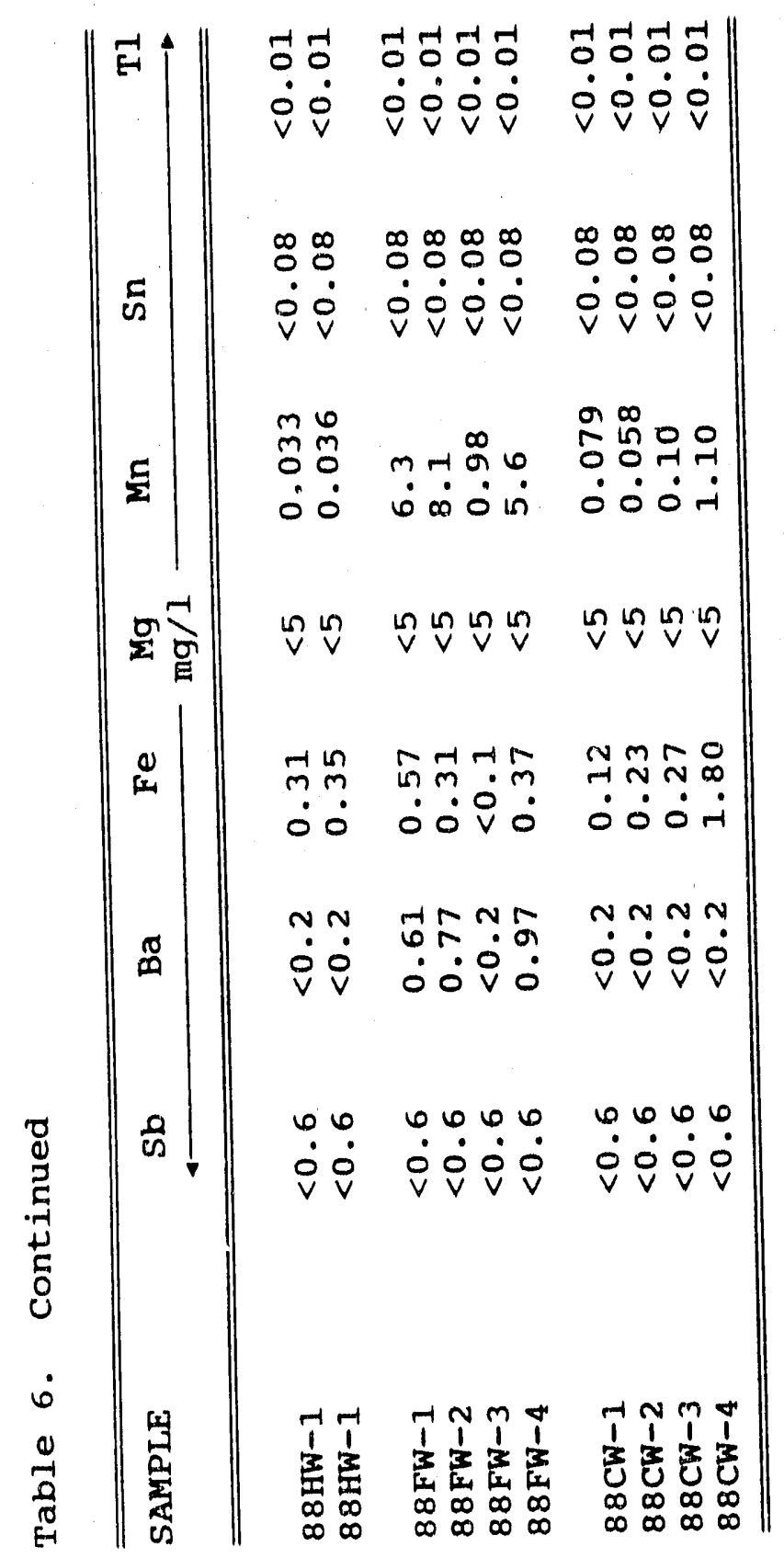




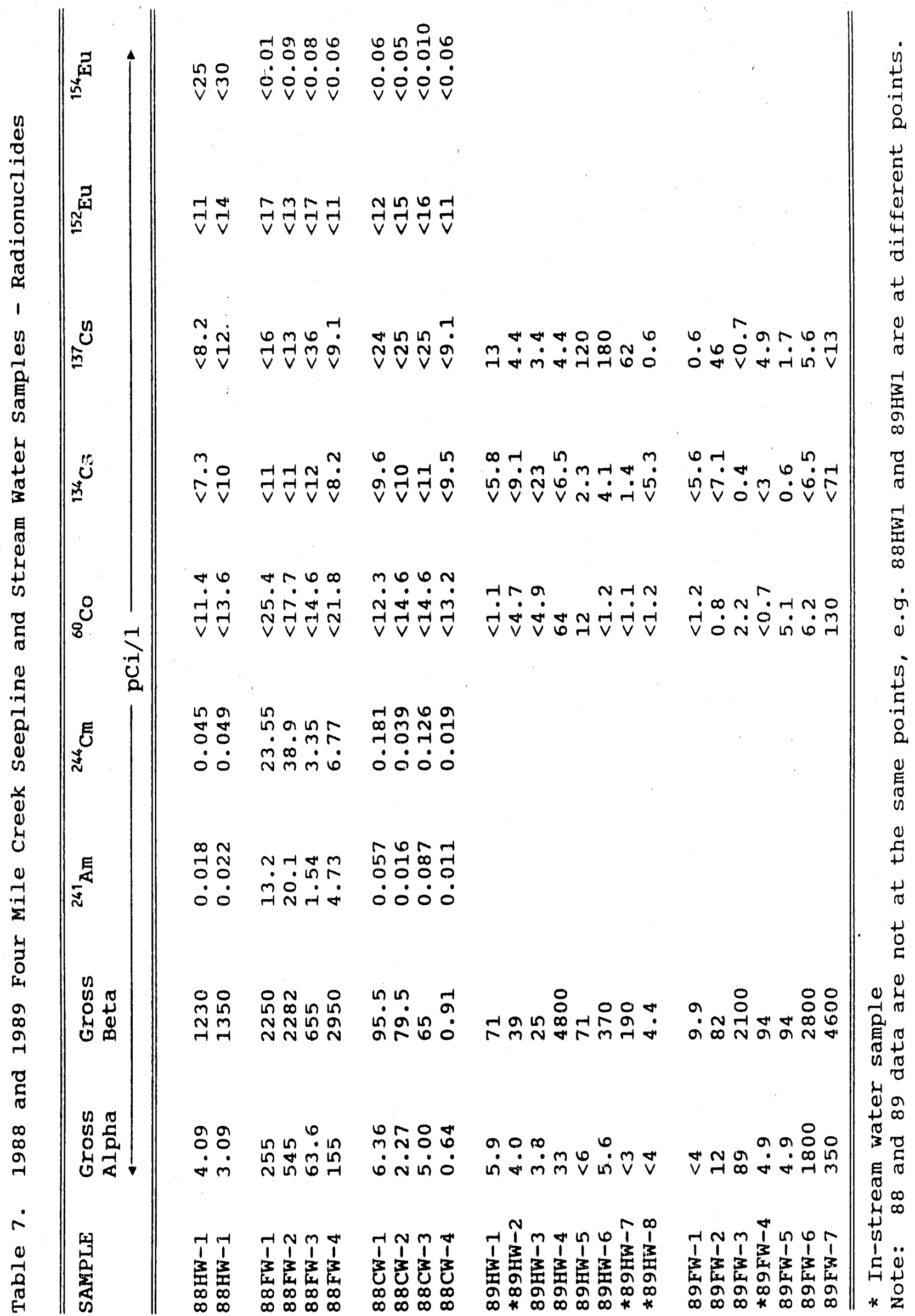




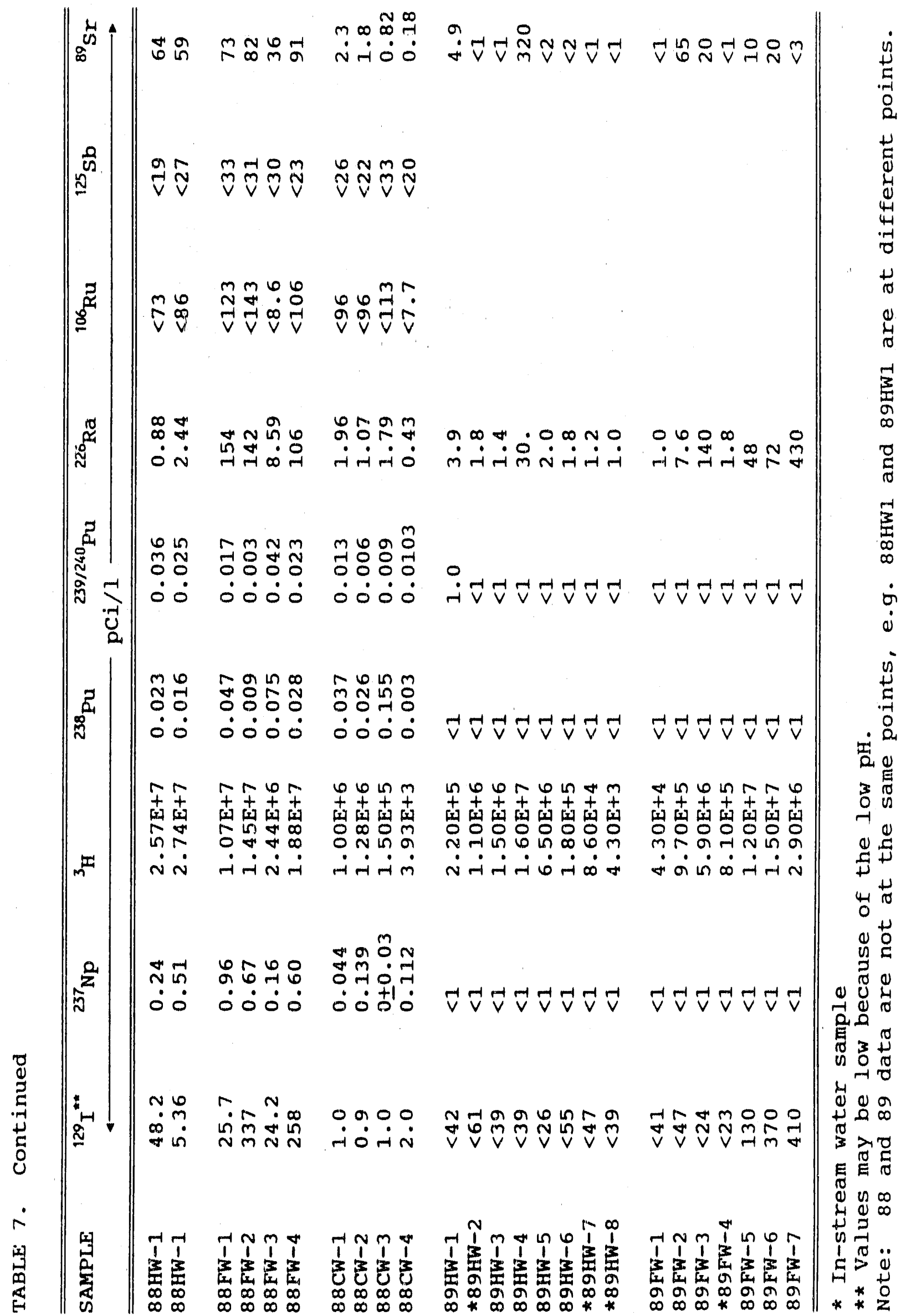




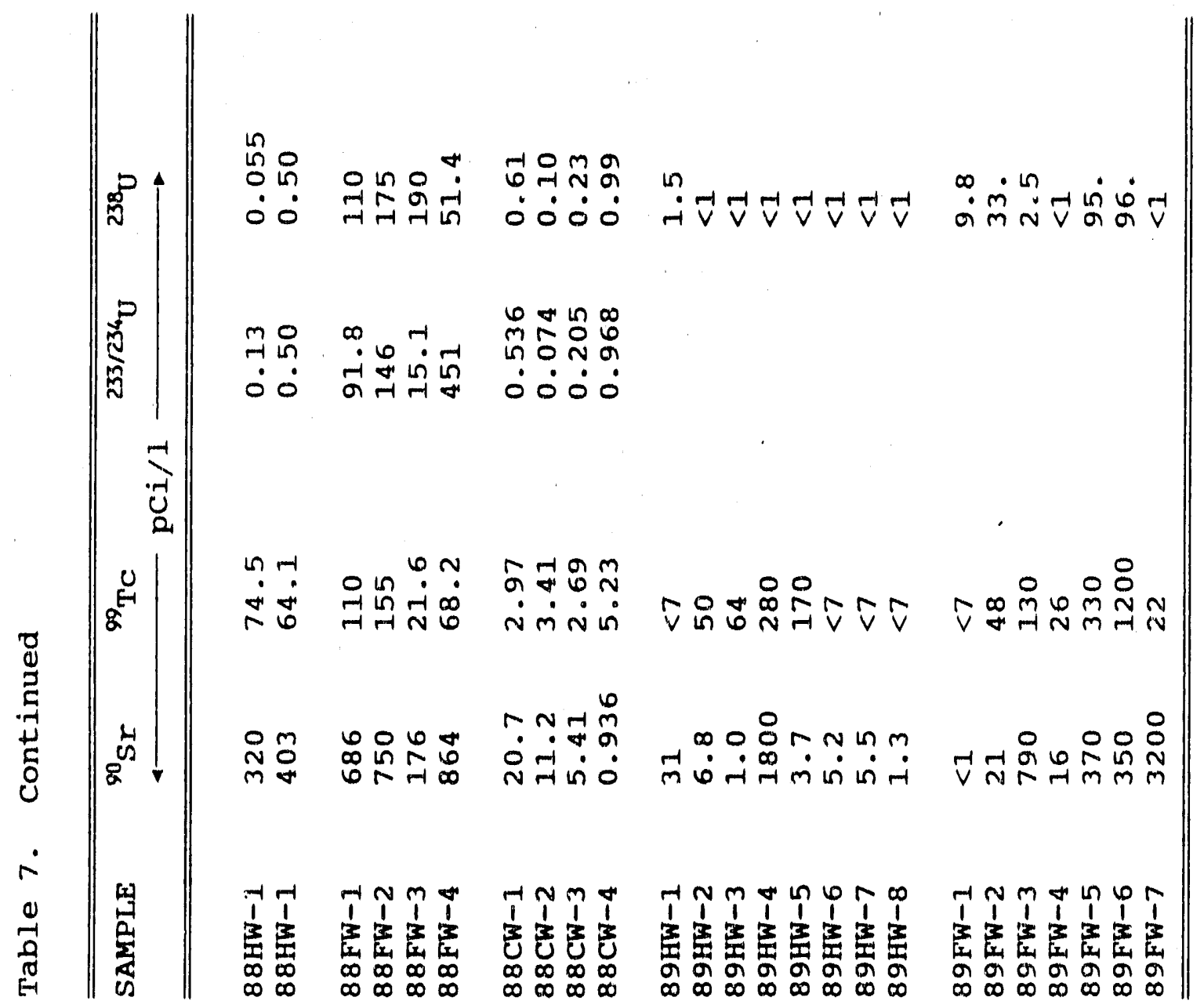




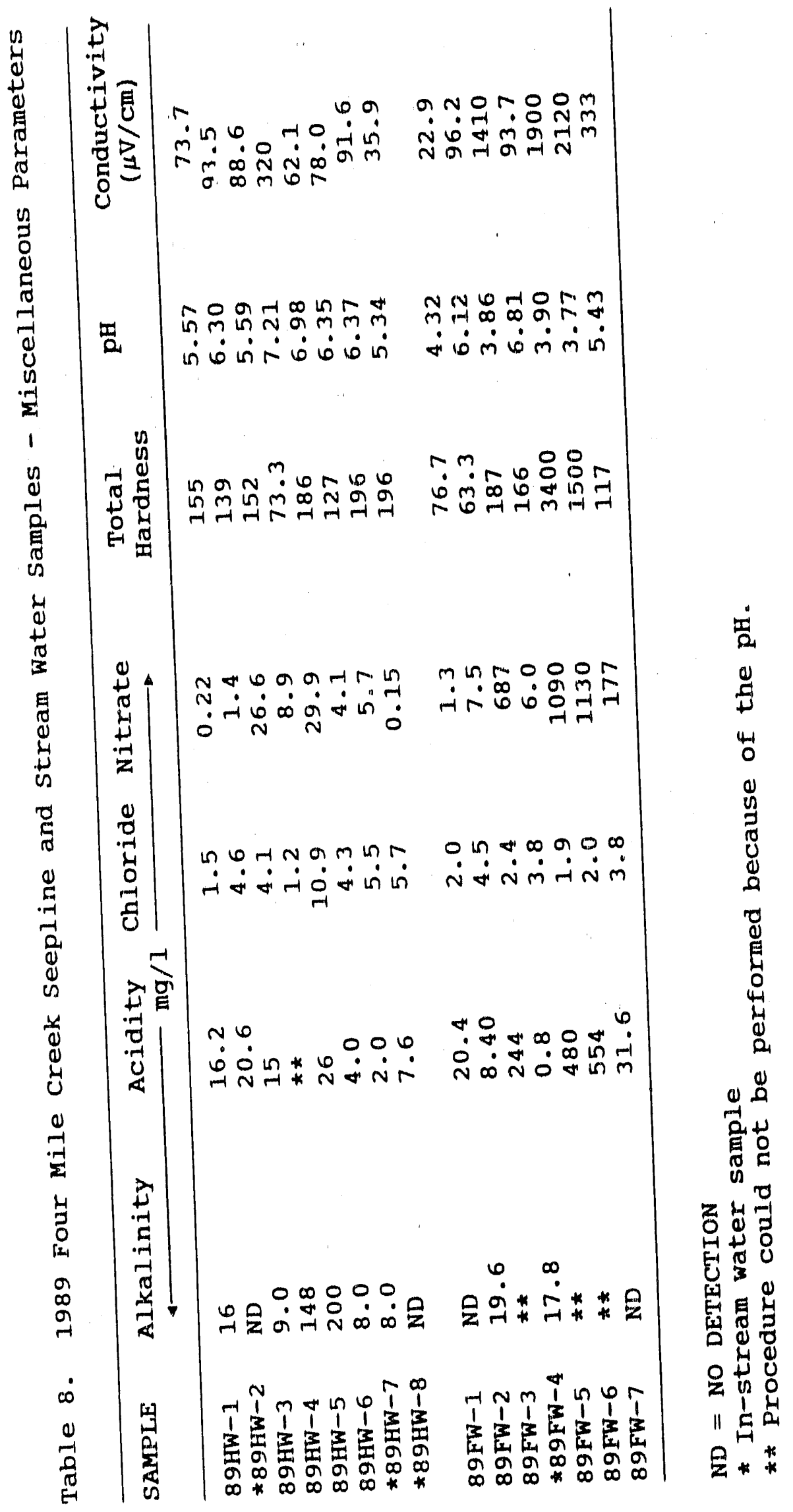




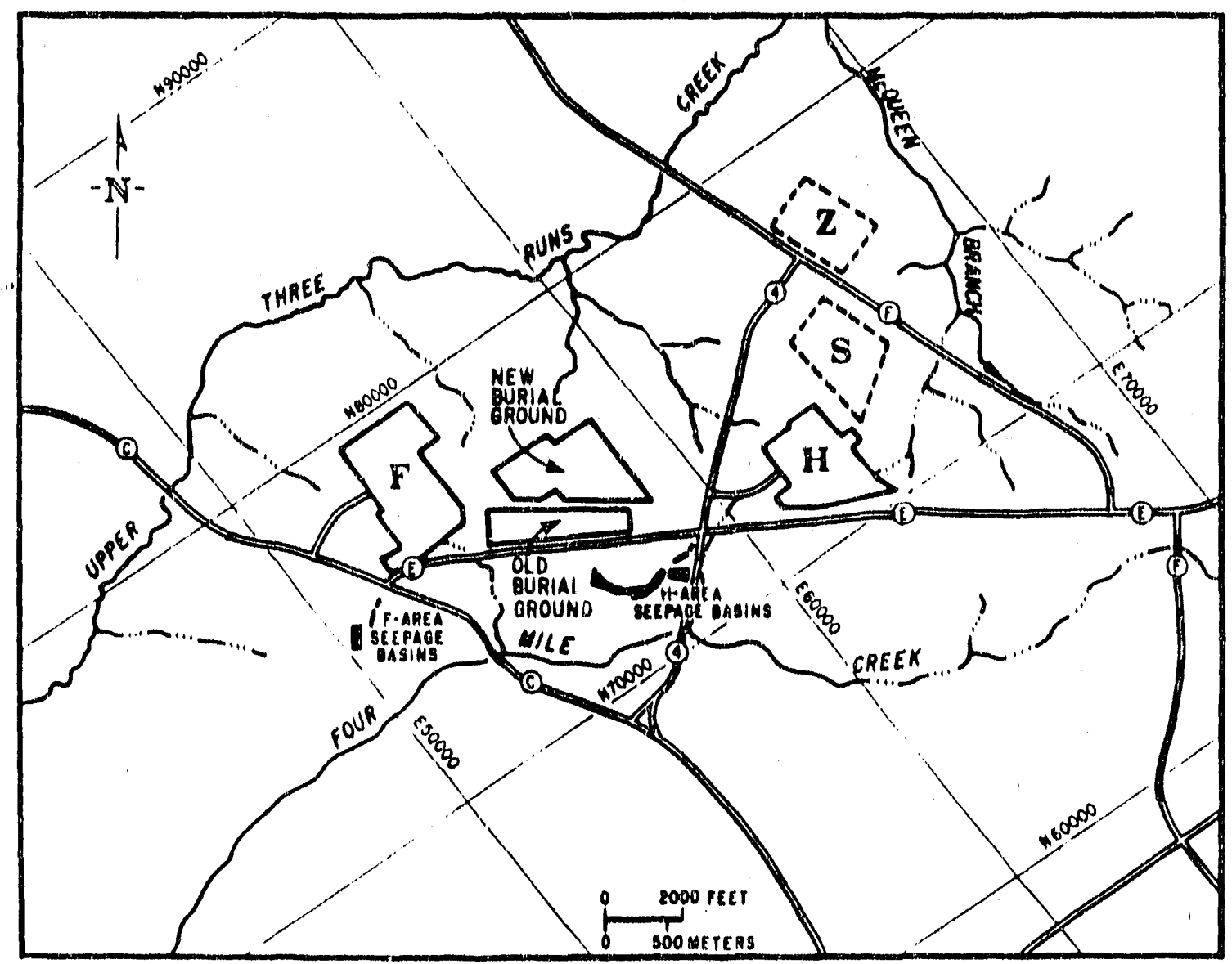

Figure 1. Location of the Separations (F\&H) Area. 


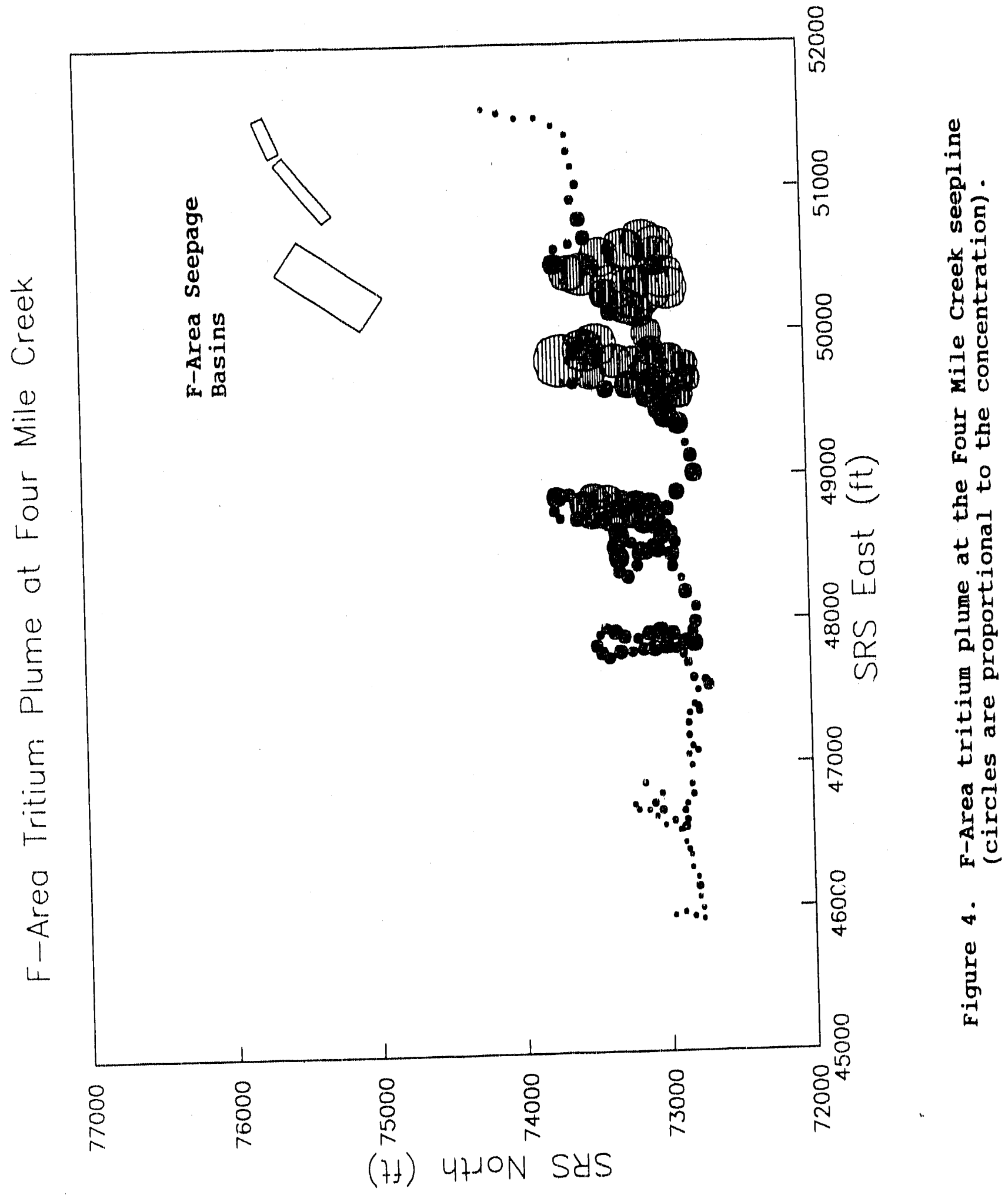




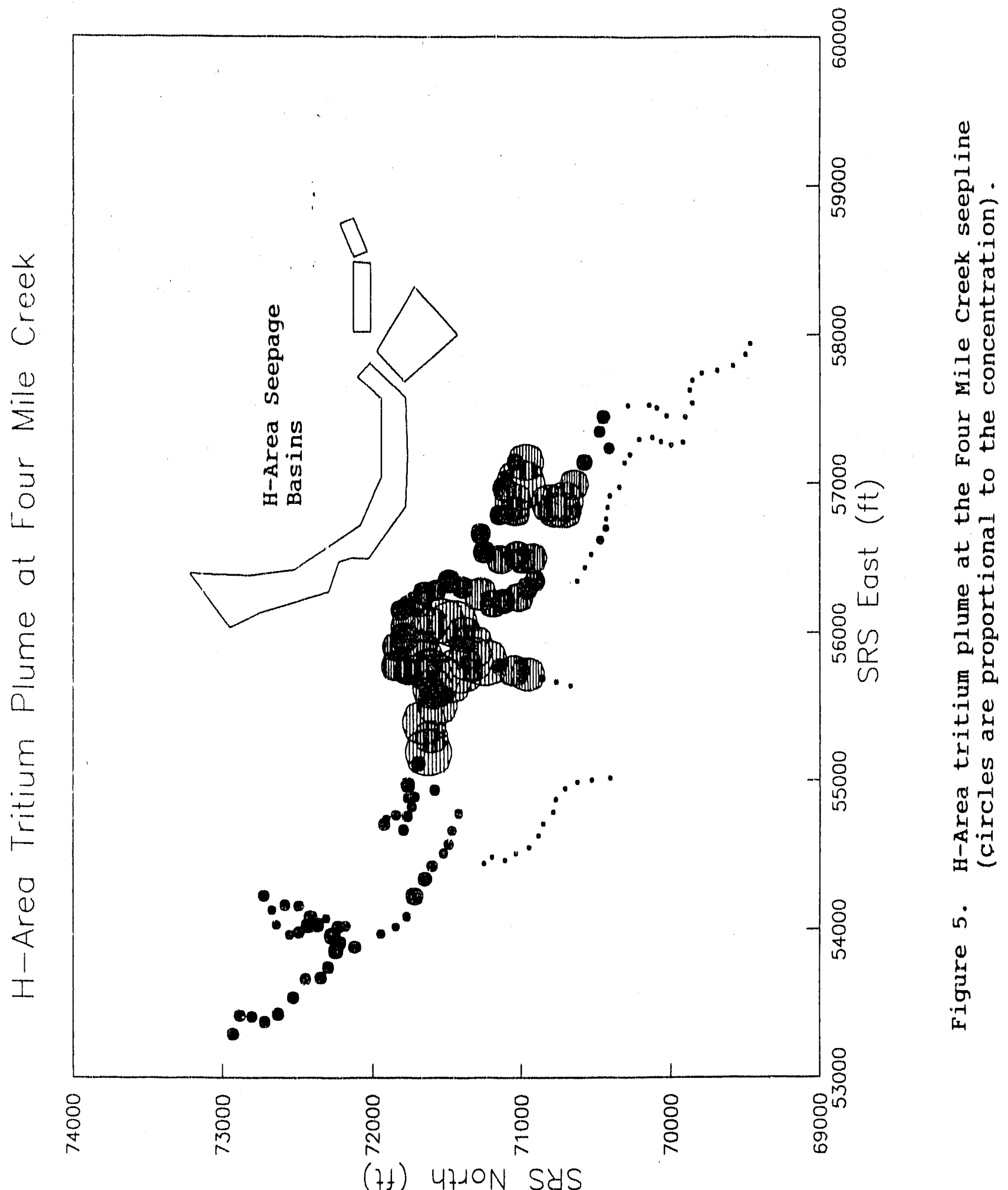




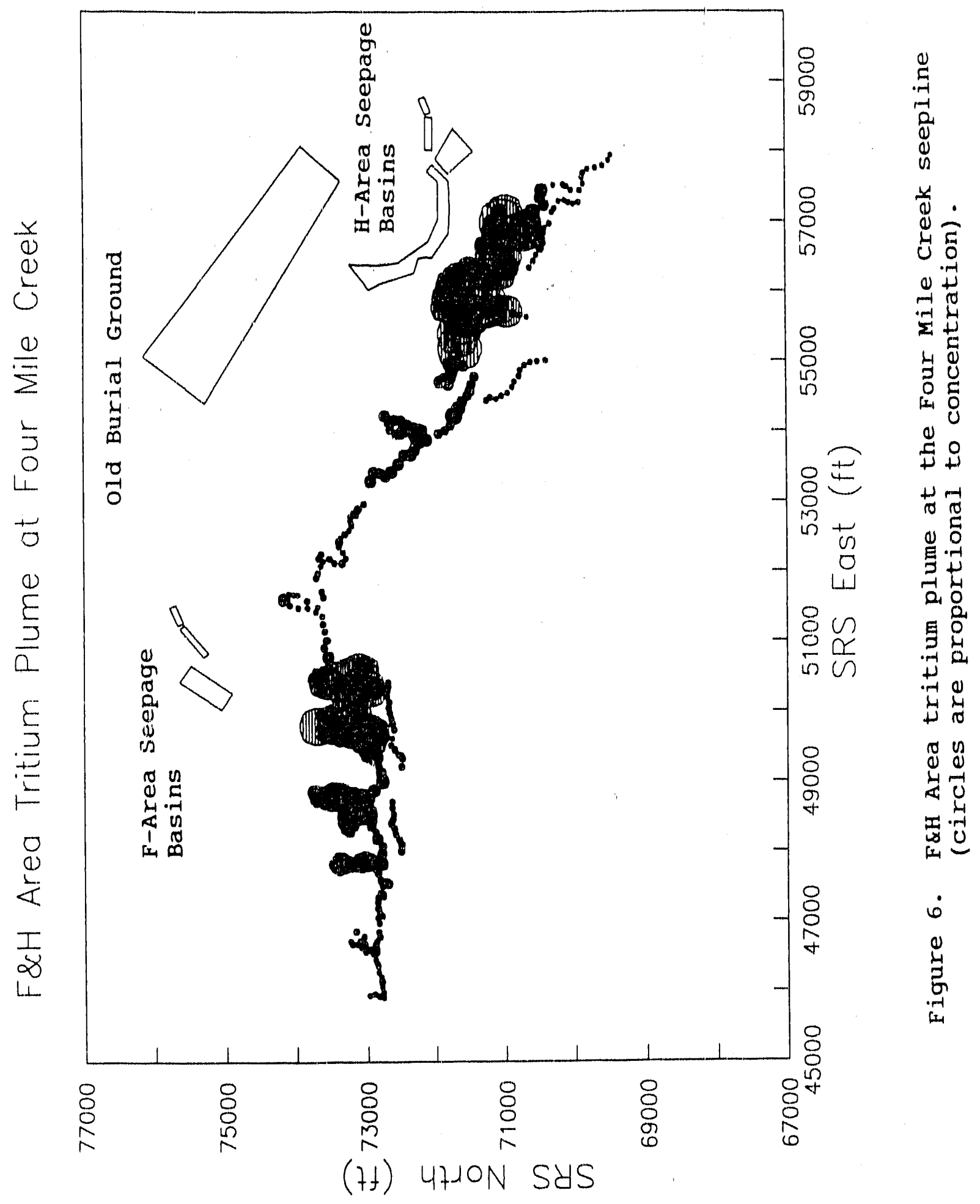




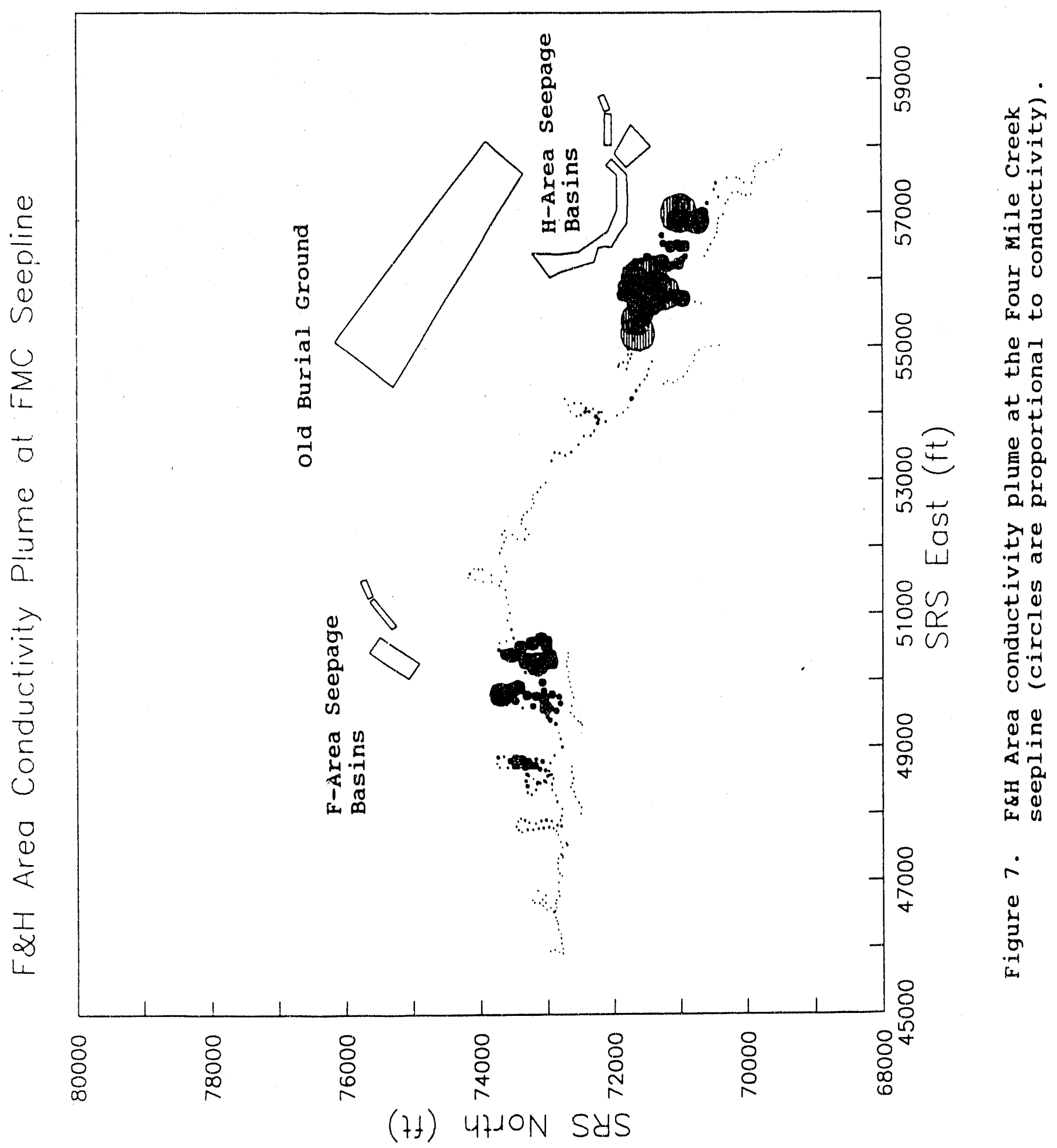




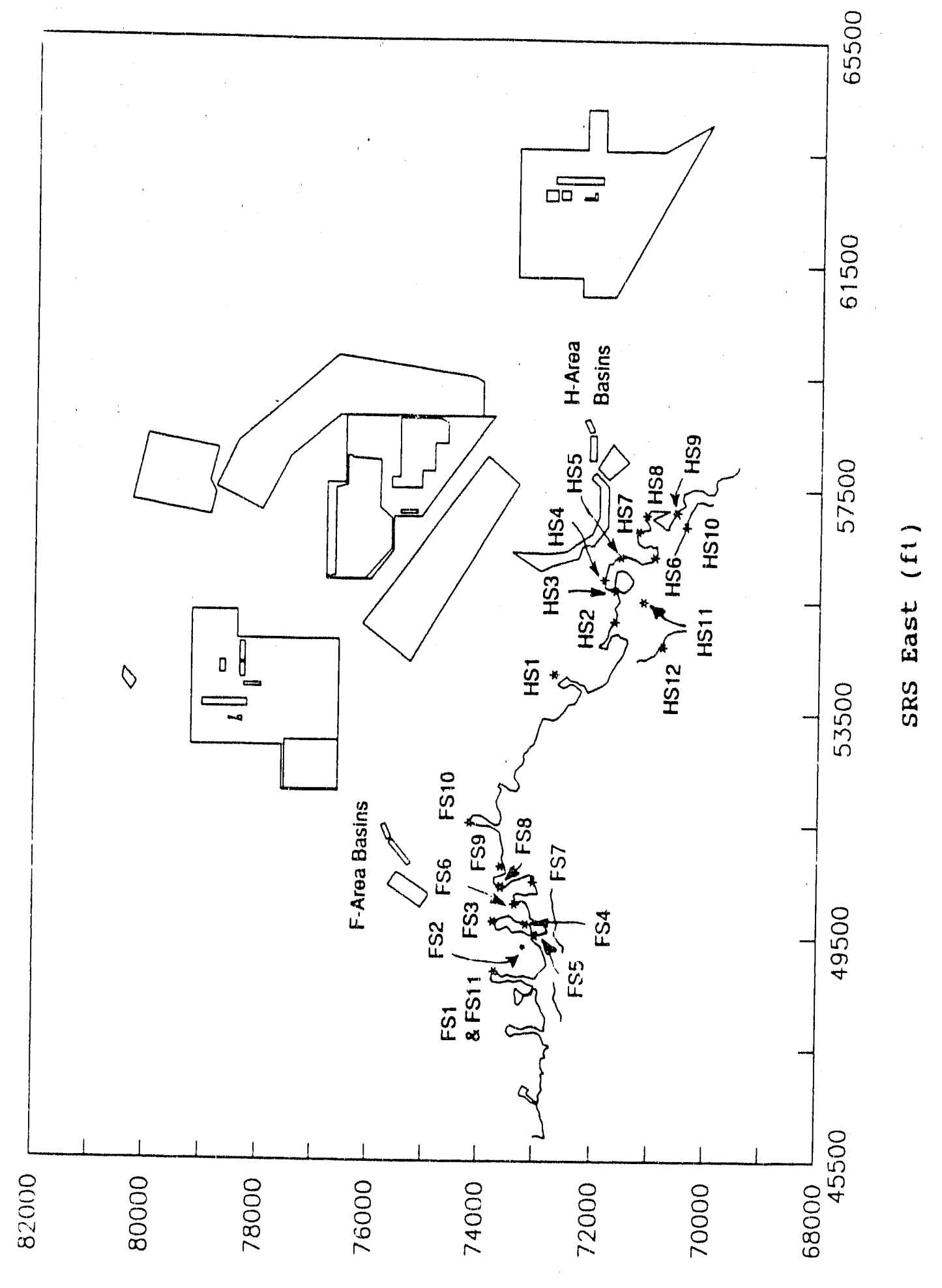

D

(7I) 47JON S4S 


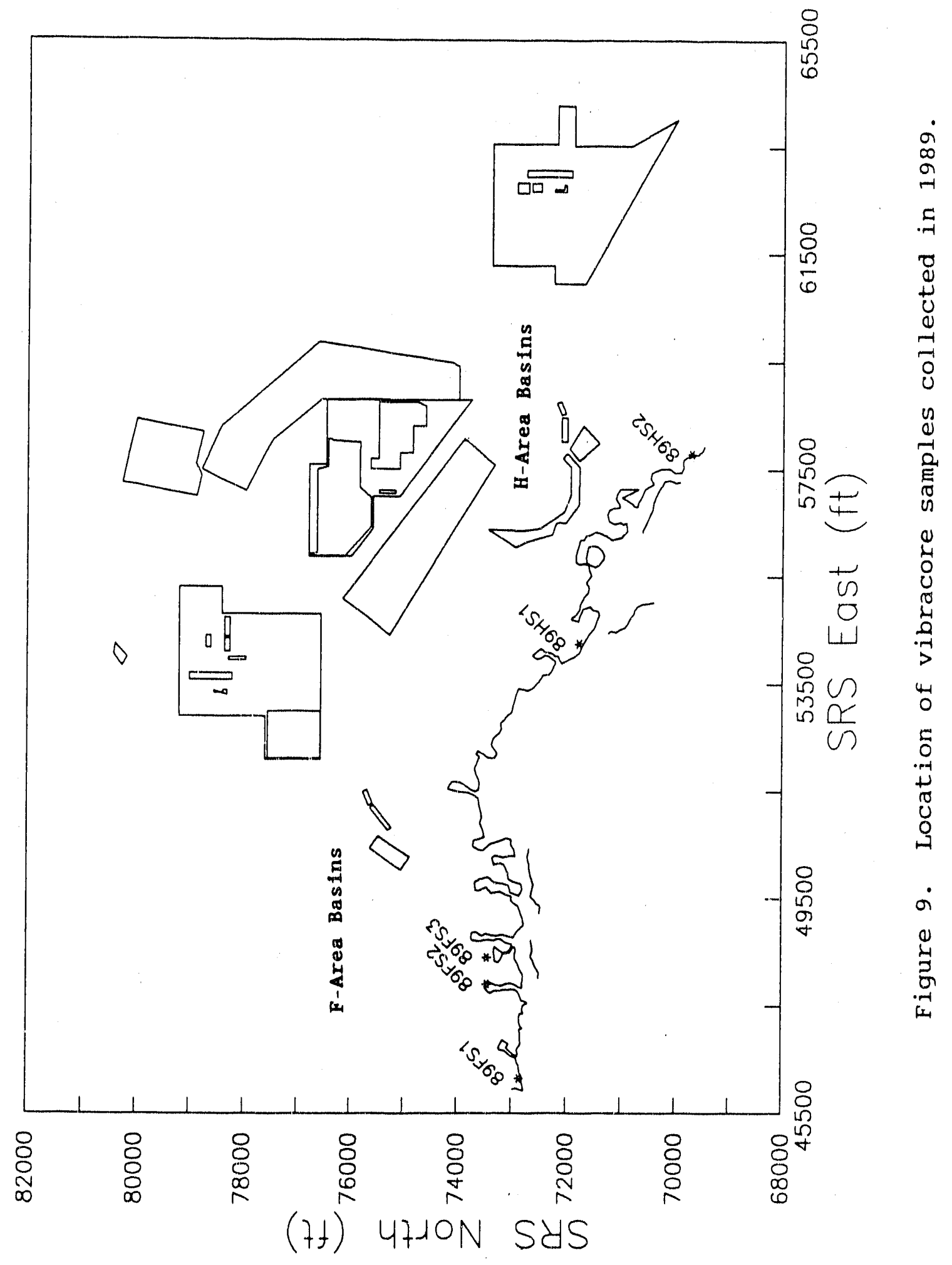




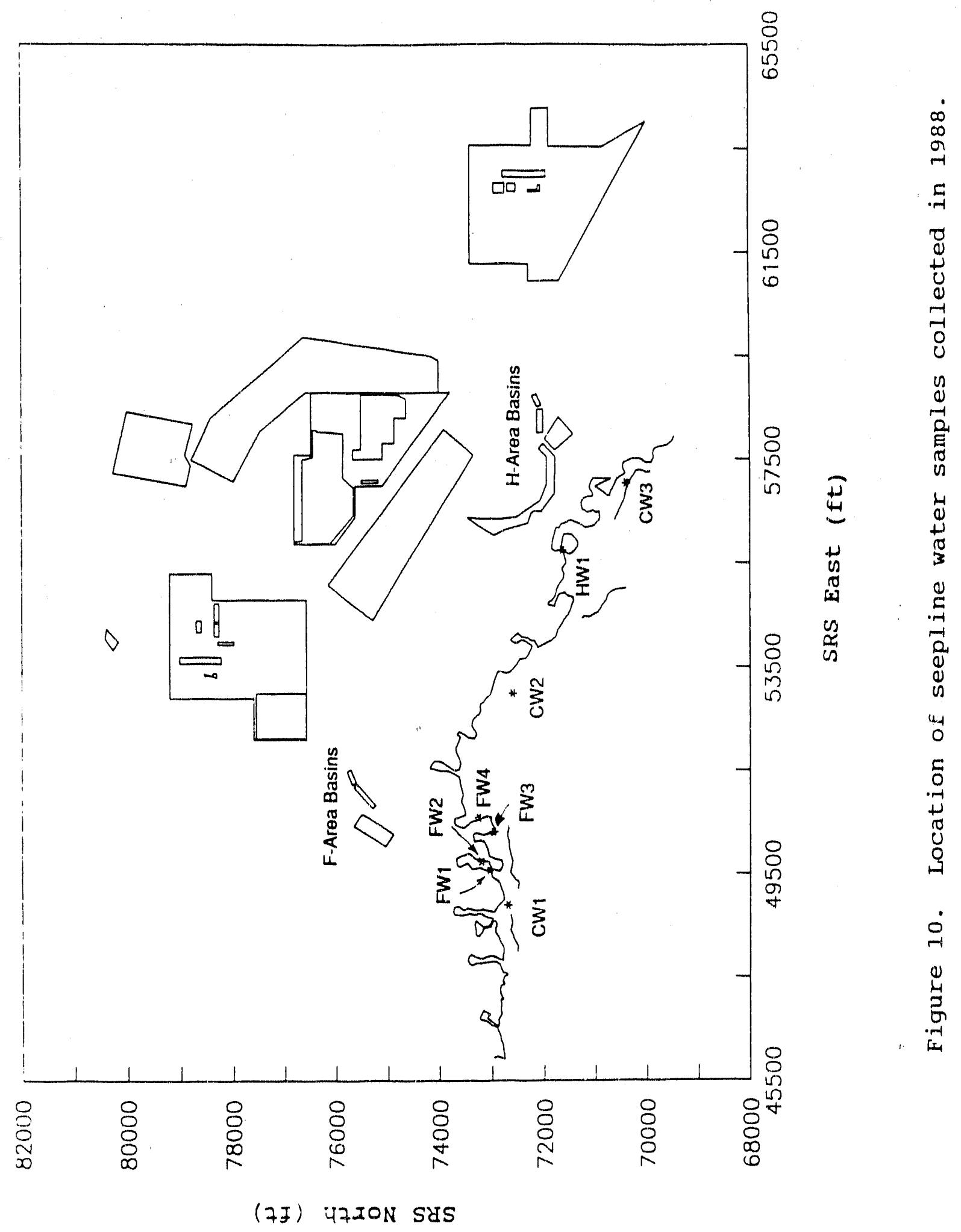




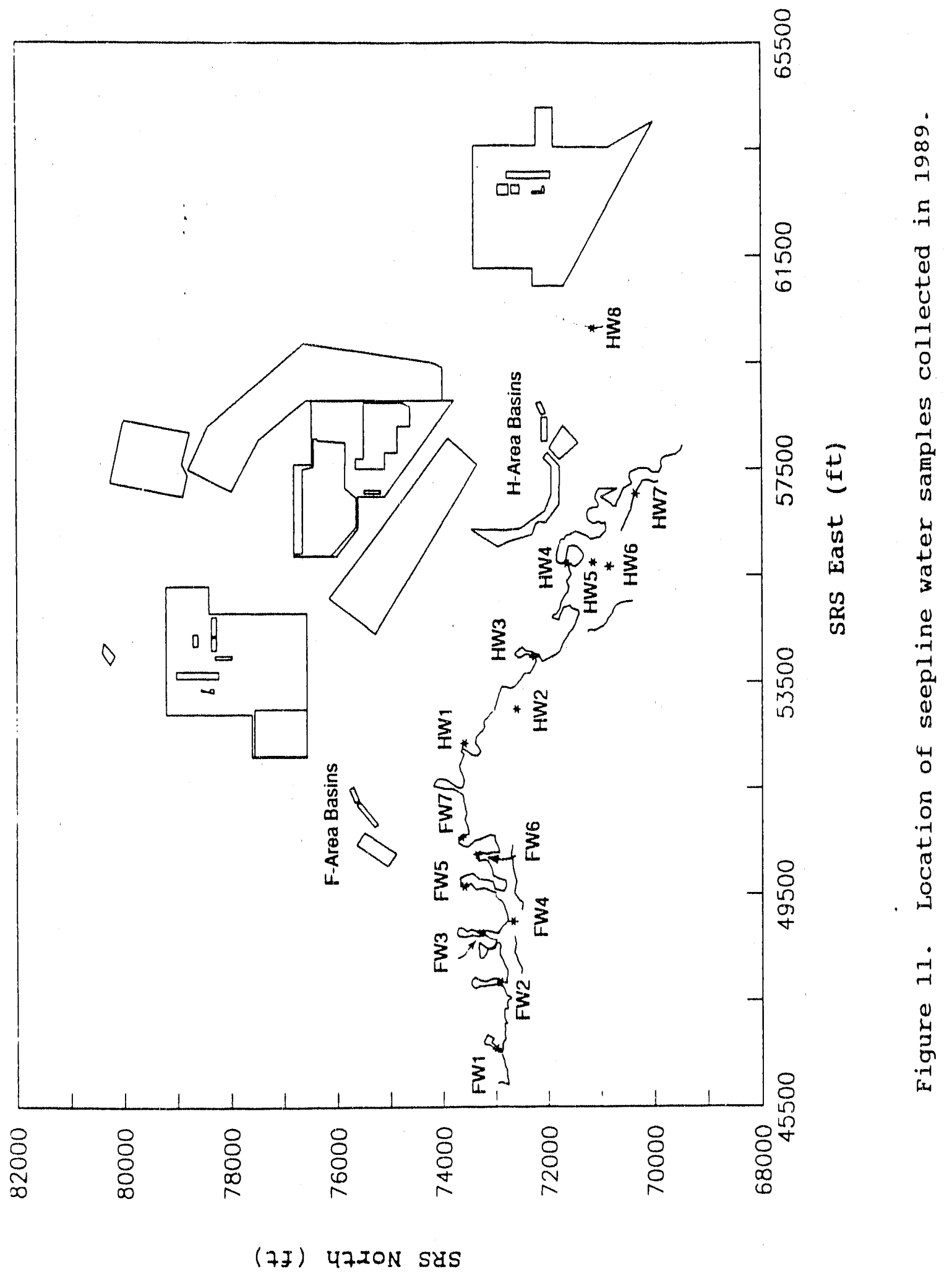




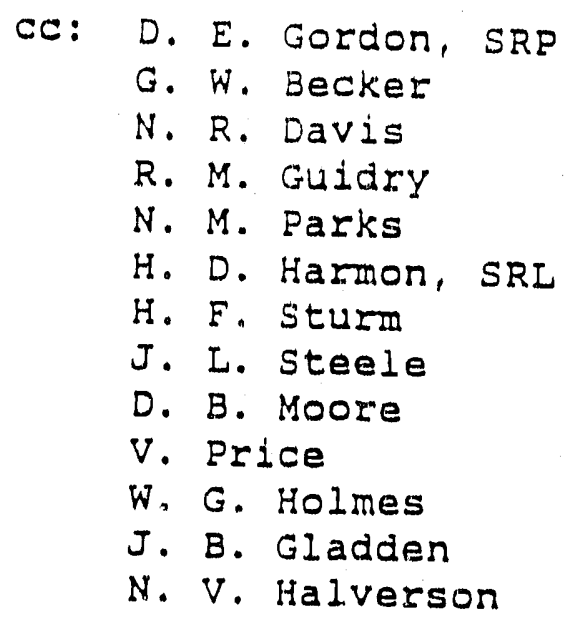

January 19, 1988

To: O. M. Morris, J. C. Corey, and G. T. Wright

From: B. B. Looney, J. E. Cantrell, and J. R. Cook

SAMPLING AND ANALYSIS OF BURPACE MATER IN THE VICINITY OF TEE F- AND E- AREA BEEPAGE BASINB

\section{Introduction and summary} Observations frow the wetlands downgradient of the
F-Area and H-Area Seepage Basins suggest that the water
cropping out along the seep line is impacting a small area
of the terrestrial/wetlands environment along Four Mile Creek.
The primary source of the water in these areas, as well as dissolved constituents in this water, are the seepage basins. The seepage basins have received large volumes of dilute liquid wastewater since the late 1950's. The primary dissolved constituents in the wastewater are hydrogen ion (low pH), nitrate and sodium. The wastewater also contains relatively low concentrations of other chemical constituents (e.g., lead and mercury). The radioactive constituent with the highest activity in the wastewater is tritium: other relatively mobile radionuclides in the wastewater include ${ }^{90} \mathrm{Sr}, 99 \mathrm{TC}, 129 \mathrm{I}$,
and $237 \mathrm{~Np}$.

The authors would like to acknowledge the assistance of two subcontracting organizations: Environmental and Chemical Sciences, New Ellenton SC, and C. B. Shedrow Environmental consultants, Ira., cslumbia, $\approx 0$. 
A key element in the technical assessment of the wetlands downgradient of the $F$ - and H- Area Seepage Basins is an analysis of the chemistry of the water cropping out at the seep lines. The bulk chemistry of this water, lncluding major cations, major anions and ionic strength will determine the chemical speciation of all of the various solutes in the water. Detailed chemical analysis of the surface water in the area will:

- assist in determining the cause of the observed vegetation stress in the area and aid in estimating the recovery period (if the stress is related to water chemistry); and

- assist in determining the nature and extent of migration for various solutes (1.e., which waste constituents have been sequestered in the subsurface and which have migrated in the water).

The overall objective of the sampling and analysis program was to collect and analyze water samples from the subject wetlands along with upstream and downstream samples from Four Mile creek.

In general, the results confirm the hypothesis that several trace metals are not migrating in the groundwater. Some constituents (primarily, sodium, ritrate and hydrogen ions) have reached the seep Iine and are affecting the bulk chemistry of the groundwater and surface water in the seep area. Several constituents (including aluminum, calcium, copper, aritm and others) appear to be leaching out of the subsurface matrix along the flow path in the low $\mathrm{pH}$ water. All of the nonradioactive constituents measured along the seep line are below primary drinking water standards except cadmium in F-Area $(0.041 \mathrm{mg} / \mathrm{L}$ maximum) and nitrate in both F-Area (546 mg/L maximum) and H-Area (36 mg/L maximum).

Samples from Four Mile creek suggest that the input of the major constituents (primarily nitrate and sodium) from the seep areas (or area outfalls) is measurable in the stream. The remaining nonradioactive constituents do not measurably impact the stream. The sampling and analysis described below represents a high quality screening of the area that will aid researchers who are planning a more robust characterization (including more sites and analysis of a range of radionuclides) in the future. 
Representative surface water samples were collected from the seep areas downgradient of the F-Area and H-Area Seepage Basins and from Four Mile Creek upstream and downstream of these seep areas. At each seep area, the precise sampling location was chosen based on a screening of the specific conductivity of the water along the seep line; the location with the highest measured specifit conductivity in each seep area was selected for detajled field and laboratory measurements (see attachment 1). The samples were collected in clean polyethylene beakers and bottles. The samples collected for metals were filtered in the field with a Nalgene Type A disposable polystyrene filter unit containing a 0.45 un equivalent pore size nitroceliulose membrane filter. The metals samples were then placed in acid washed bottles and preserved with $2 \mathrm{ml}$ of nitric acid. Mercury analyses were performed on both the filtered and unfiltered (bulk) samples. Also, both flitered and nonfiltered samples for all metals were collected in Four mile creek downstream of the F-Area seepage Basins. Field notes and a description of each sample location are sumarized.in Table 1 (a complete listing is in Attachment 1). Figure 1 is a map showing the locations of the sampling sites.

\section{Rosults}

The various analyses are sumarized in Tables 2 and 3 . The complete data set is presented in Attachment 2. The median and maximum values for each location, along with the number of samples collected 1 - presented in Table 2. Table 3 shows the relationship between the filtered and unfiltered samples collected downstream of the F-Area Seepage Basins.

\section{Discussion}

The primary goals associated with the interpretation of these chemical data, along with the ecological and hydrogeological data, are to: (1) evaluate the potential effects of seepage basin operation on the downgradient receptors, (2) quantify the expected recovery of these areas following closure, and ( 3 ) determine the nature and extent of migration of the various solutes in the subsurface system. A preliminary discussion of these topics based on the chemical screening of surface waters in the vicinity of the seepage basins is presented below. 
The coneentrations of whe various constifuents in the seep areas downgradient of the seepage basing suggest that basin operarions have significantiy affected these areas. The concentrations of several waste constituents (primariiy sodium, nitzace, and hydrogen ion) are elevated in the surface water along the seep line. This finding is consistent with the findings of Horton and Carothers (1974) in which elevated concentrations of nitrate were reported. Horton and Carothers (1974) reported a maximum seep line concentrarion of $217 \mathrm{mg} / \mathrm{L}$ (as $N$ ) in $F$ Area and $242 \mathrm{mg} / \mathrm{L}$ (as $\mathrm{N}$ ) in $\mathrm{H}$ Area. The data in Attachment 2 show that current levels at the same approximate locations are 174 to $546 \mathrm{mg} / \mathrm{L}$ (as N) in F Area and 22.9 to $36.3 \mathrm{mg} / \mathrm{L}$ (as N) in 4 Area. The appearance of tritium (Reichert, 1962, 1968), 99 TC, 129 I (Anderson, 1980; Kantelo, 1987), and nitrate in the water along the seep line in the mid sixties and early seventies and relatively constant concentrations thereafter suggests that the front of these constituents (along with sodium) is relatively mobile and that the system has approximately reached a steady state. Horton and Carothers (1974) concluded the following in regard to nitrate in the groundwater and Four Mile Creek:

- The territory between the seepage basins and Four Mile creek can not be released for unrestricted use until the nitrate has been reduced substantially. [Based on flow rate considerations, ] this is estimated to require a decade or more after discharge of nitrate to the basins ceases. Such variables as groundwater flow rate when it is not influenced by water from the basins and the biological rate of nitrate removal will influence this time period.

- [Based on an analysis of stream concentrations and outfall concentrations, ] the seepage basins are the major contributor of nitrate to the creek. The H seepage basins contributed nitrate at a rate of 88 tons/yr and the $F$ seepage basins 65 tons/year during the sampling period (August through september 1973).

Interpretation of the current chemical data set from the groundwater, seep Iine and Four Mlle creek is generally consistent with that developed primarily for nitrate by Horton and carothers (1974). Thus, relatively mobile constituents will flush through the subsurface system. During this period, elevated concentrations will exist in the surface seeps; following this period, the concentrations in the groundwater and surface waters in the vicinity should decline. The low concentrations of the various hazardous constituents (e.g., lead and mercury) suggests that these are not factors in the observed iegetaニiこn stzsss. The high conductivity, the high sodium, 
nitrate, and aluminum concentrations, and the flooding of previously dry areas caused by the basins are likely the factors influencing the vegetation in the affected areas.

As a preliminary means to determine the significanca of the current concentrations, the levels of the various constituents along the seep 1 ine and in Four Mile Creek were compared to primary drinking water standards. All of the nonradioactive constituents measured along the seep line are below primary drinking water stanciards excopt cadmium in F-Area $(0.041 \mathrm{mg} / \mathrm{I}$ maximum) and nitrate in both F-Area (546 mg/L maximum) and $H$-Area $(36 \mathrm{mg} / \mathrm{L}$ maximum). All chemical constituents are below their respective primary drinking water standards in Four Mile Creek. A comparison of the filtered and unfiltered samples from Four Mile Creek downstream of F Area (note that this site is downstream of both facilities indicates that particles play a minor role in transporting metals at this location.

The analyses of the upstream and downstream samples from each area suggest that the following parameters are statistically impacted (i.e., using a t-test and the $t$ values for $95 \%$ confidence, the concentration was significantly higher downstream):

H Area - conductivity, total dissolved solids, Mitrate, phosphate, sodium, and potassium

F Area - conductivity, total dissolved solids, sodium, manganese and possibly cadmium.

The remaining constituents were either not statistically elevated, $0^{\gamma}$ the concentration in the strearn was higher than the seep line, or the concentration was lower downstream than upstream. These data suggest that the basins may contribute measurable quantities of the identified constituents to the stream. Note, however, that several outfalls enter Four Mile creek in the subject area. The outfalls between the H-Area upstream and downstream samples include HB, H9 (abandoned), HII, and H12. The outfalls between the F-Area upstream and downstream samples include $F 8$, and FIO.

The relative subsurface mobility of the various constituents originally present in the wastewater sent to the seepage basins can be assessed in a preliminary fashion by calculating a ratio betrieen the measured concentration in water at the seep line and the measured concentracion of the wastewacer sent to the basin. This "transport factor" will vary as a function of the mechanisms operating in the flow path. For example, if a constituent is sorbed to the subsurface matrix, chemically neutralized, biologically transformed, or utilized as a nutrient, it will be 
removed from the flowho water and the concentratzon idit. be

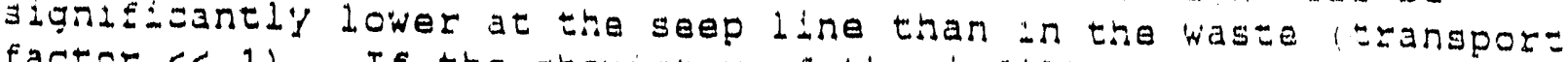
factor $<1)$. If the chemistry of the infiltraring wastewater results in signiflcant leaching of a constituent from the subsurface materlals, the concentration at the seep will be higher than the original wastewater (transport factor $>1$ ). If the rransport factor is near 1 for a constituent, it may be travoling with the water (or may be governed by a comblnation of sorption and leaching).

Table 4 summarizes the transport factors calculated using the median seep line concentrations and the wastewater concentrations reported in K.111ian et al. (1987a, 1987b). In general.

- mercury and chromium appear to be sequestered by the soil.

- sulfate and sodium appear to move with the groundwater.

- some constituents appear to have reached the seep area in $F$ Area $(\mathrm{pH}=3.8)$ but not in H Area $(\mathrm{pH}=6.4)$. These include zine and possibly lead.

- several constituents appear to be leached from the matrix in $F$ Area but not in H Area. These include silica, calcium, barium, copper, aluminum, manganese, ard magnesium.

Note that this approach is not definitive, and further study of the moblilty of solutes as a function of solution and soil properties is recommended.

The sampling and analysis of surface water samples in the vicinity of the F- and H-Area Seepage Basins was carried out in a careful manner and the results provide a high quality screening of the conditions in the area. These data, the on-going analysis of radionuclides in the samples, and future studies will assist SRP and SRI in developing a technically sound closure plan that protects human health and the environment. 
Tablo 1

sumary of F1eld Masuraments and Obaervations

H-Area Seepage Basins Samples

Seep Area--Sample numbers FH 002, FH 008, and FH 014

Samples collected along the seep line

downgradient of the H Area Seepage Basins--south

of Basin H4. The water was turbid containing leaf

Iitter.

H-Area Upstream--Sample numbers FH 003, FH 009, and FH 015

-

Samples collected approximately 50 m downstream

of Road $F$ on Four Mile creek. The stream in this

area is small but well defined. Upstream (across the road) is a marshy wetland area. The samples were brow colored and contained some solids.

H-Area Downstream--Sample numbers FH 004, FH 010, and FH 016

Samples collected approximately 100 m upstream of Road $C$ on Four Mile creek. The area has a deep clean channel, a high flow and low solids. 
Table 1 (contigued)

sumary of Flold Measuraments and Observations

F-Area Seepage Basirs Samples

Seep Area--Sample numbers FH 001, FH 007, and FH 013

Samples collected along the seep line

downgradient of the $F$ Area seepage Basins--east of well cluster FSB 79. Water was turbid containing leaf litter and a large crop of fliamentous - algae.

F-Area Lpstream--sample numbers FH 005, FH 011, and FH 017

Samples collected approximately 100 m downstream of Road $C$ on Four Mile creek. The area is very mucky and marshy and breaks into many small channels in this area. One of the northern channels was selected for sampling. The water was turbid containing flocculant brown mud.

F-Area Downstream--Sample numbars FH 006, FH 018

Samples collected approximately $10 \mathrm{~m}$ downstream of F-Area steam line road on Four Mile creek. The area has a deep clean channel, a high flow and the least solids of any site. Three additional samples were collected at this site (FH 012, FH 019, and FH 020); the additional samples were collected without filtering the metals subsample. 
Tabla 2

Sumary of the chemical Analyses of the surface water in the viciaity of the F- and H-Area Beopage Bagins

Surtace Water Oata in the Vicintry of the H.Area Sequage Basins

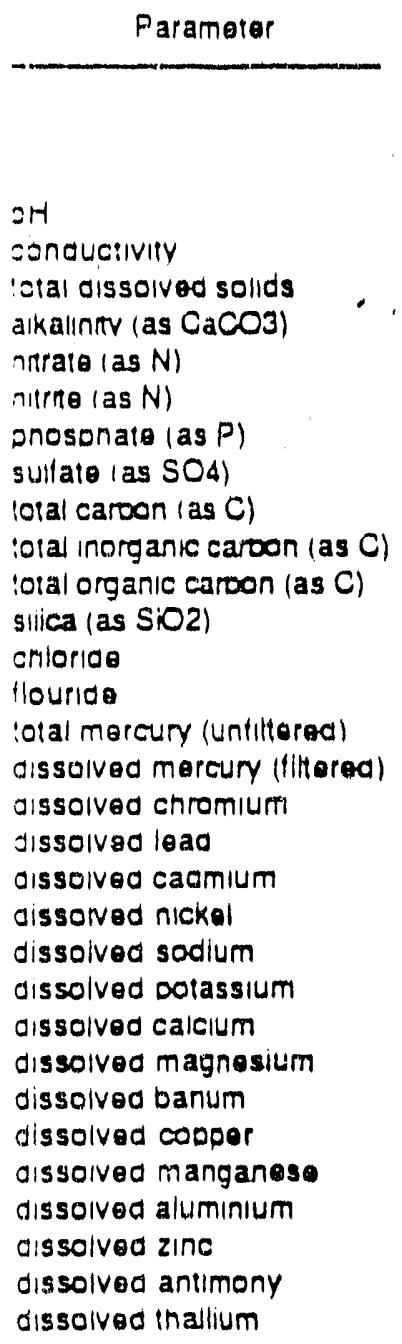

\begin{tabular}{|c|c|c|c|}
\hline \multirow[t]{2}{*}{ Units } & \multicolumn{3}{|c|}{ Soro Line } \\
\hline & $N$ & iredian & Maximum \\
\hline - $\log (\mathrm{H})$ & 6 & 6.4 & $N A$ \\
\hline WS/cm & 6 & 293 & 348 \\
\hline $\mathrm{m} / \mathrm{g} / \mathrm{L}$ & 3 & 278 & 310 \\
\hline $\mathrm{mg} / \mathrm{L}$ & 3 & 27.9 & 90.3 \\
\hline$m g / h$ & 3 & 28.5 & 36.3 \\
\hline$m g / L$ & 3 & 0.031 & 0.033 \\
\hline $\mathrm{mg} / \mathrm{L}$ & 3 & 1.86 & 6.22 \\
\hline $\mathrm{mg} / \mathrm{L}$ & 3 & 5.4 & 5.7 \\
\hline $\mathrm{mg} / \mathrm{L}$ & 3 & 25.9 & 35.8 \\
\hline $\mathrm{mg} / \mathrm{L}$ & 3 & 11.7 & 16.0 \\
\hline$m g / L$ & 3 & 14.2 & 19.8 \\
\hline $\mathrm{mg} / \mathrm{L}$ & 3 & 5.5 & 5.6 \\
\hline$m g / L$ & 3 & 5.3 & 6.2 \\
\hline $\mathrm{mg} / \mathrm{L}$ & 3 & $<0.1$ & $<0.1$ \\
\hline $\mathrm{mg} / \mathrm{L}$ & 3 & $<0.0009$ & 0.0001 \\
\hline $\mathrm{mg} / \mathrm{L}$ & 3 & $<0.0001$ & 0.00015 \\
\hline$m g / L$ & 3 & $<0.001$ & $<0.001$ \\
\hline $\mathrm{mg} / \mathrm{L}$ & 3 & $<0.003$ & 0.003 \\
\hline $\mathrm{mg} / \mathrm{h}$ & 3 & $<0.0001$ & $<0.0001$ \\
\hline$m g / L$ & 3 & $<0.05$ & $<0.05$ \\
\hline $\mathrm{mg} / \mathrm{L}$ & 3 & 68.4 & 69.3 \\
\hline $\mathrm{mg} / \mathrm{l}$ & 3 & 1.85 & 1.86 \\
\hline$m g / L$ & 3 & 1.67 & 1.8 \\
\hline$m g / L$ & 3 & 1.43 & 1.48 \\
\hline$m g / L$ & 3 & $<0.05$ & $<0.05$ \\
\hline$m g / L$ & 3 & $<0.05$ & $<0.05$ \\
\hline$m g / t$ & 3 & 0.145 & 0.164 \\
\hline $\mathrm{mg} / \mathrm{L}$ & 3 & 0.963 & 0.183 \\
\hline$m g / L$ & 3 & $<0.02$ & $<0.02$ \\
\hline$m g / L$ & 3 & $<0.01$ & $<0.01$ \\
\hline$m g / L$ & 3 & $<0.004$ & $<0.004$ \\
\hline
\end{tabular}

Four Mile Creak Uosiream Four Mile Creek Downstream

$N$ Median Maximum is Median Maximum

$\begin{array}{rrrr}4 & 5.0 & \text { i.A } & 4 \\ 4 & 30 & 39 & 4 \\ 3 & 39 & 42 & 3 \\ 3 & <1.0 & <1.0 & 3 \\ 3 & 0.04 & 0.19 & 3 \\ 3 & 0.019 & 0.024 & 3 \\ 3 & 0.011 & 0.014 & 3 \\ 3 & 1.8 & 2.2 & 3 \\ 3 & 8.3 & 8.9 & 3 \\ 3 & 4.0 & 4.4 & 3 \\ 3 & 4.3 & 5.6 & 3 \\ 3 & 10 & 10 & 3 \\ 3 & 6.5 & 6.6 & 3 \\ 3 & <0.1 & <0.1 & 3\end{array}$

$\begin{array}{rr}6.6 & N A \\ 90 & 32 \\ 72 & 75 \\ 16.1 & 16.2 \\ 1.15 & 1.2 \\ 0.005 & 0.005 \\ 0.023 & 0.023 \\ 10.5 & 10.8 \\ 0.2 & 8.3 \\ 4.6 & 6.8 \\ 3.6 & 3.7 \\ 9.9 & 10.2 \\ 5.1 & 5.3\end{array}$

$<0.0001<0.0001$

$<0.1$

$<0.1$

$<0.00010 .000133<0.0001$

0.00024

$<0.001<0.0013<0.001$

0.00012

$<0.003<0.003 \quad 3<0.003$

$<0.001$

$<0.00010 .000113<0.0001$

$<0.003$

$<0.05<0.053$

$<0.05$

$<0.0009$

0.05

$<0.05$

$\begin{array}{lll}2.8 & 2.8 \quad 3\end{array}$

$<0.20<0.20 \quad 3$

1.57

13.2

$\begin{array}{lll}1.45 & 1.47 \quad 3\end{array}$

4.03

1.61

4.12
$\begin{array}{lll}0.55 & 0.55 & 3\end{array}$
0.66

$<0.05<0.053$

$<0.05<0.05$

$\begin{array}{lll}0.118 & 0.119 & 3\end{array}$

0.69

$<0.05$

$<0.05$

$<0.05$

0.083

$<0.05$

$0.06 \quad 0.065 \quad 3$

$<0.05$

0.085

$<0.02<0.02 \quad 3$

$<0.02$

$<0.05$

$<0.01<0.013<<0.01$

$<0.02$

$<0.004<0.0043<0.004<0.004$

$<0.01$ 
Table 2 (contiaued)

Sumary of the chomical Analyses of the surface water in the Viciaity of tbo P- and E- Area seepage Basias

Eurlace Water Data in ine Vicinity of the F.Area Secoage Basins

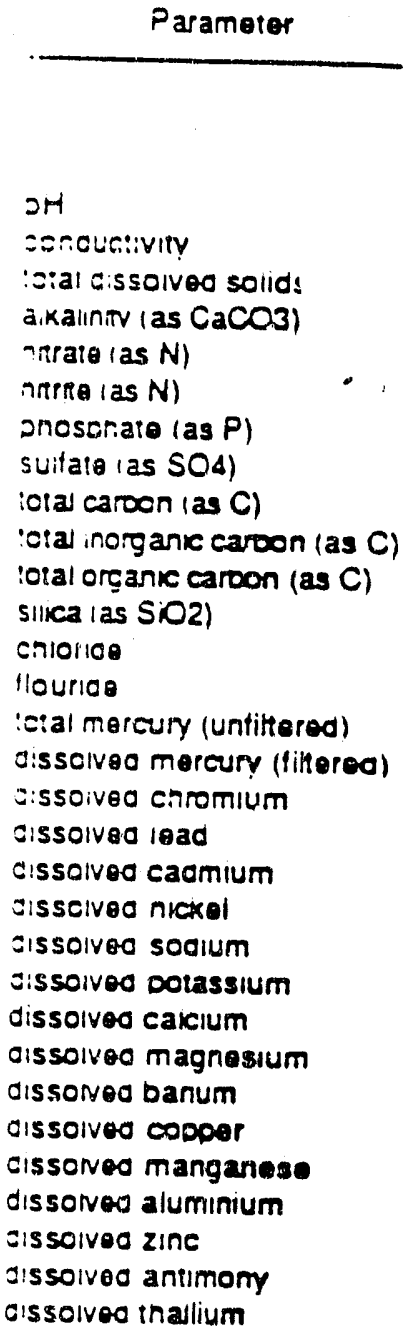

Four Mile Creok Uostream Four Mile Creok Downstream

\begin{tabular}{|c|c|c|c|}
\hline Units & & Soeo L & Lne \\
\hline & $N$ & Moolan & Maximum \\
\hline$\cdot \log (\mathrm{H})$ & 6 & 3.8 & $N A$ \\
\hline us/cm & 6 & 1295 & 1760 \\
\hline $\mathrm{mg} / \mathrm{h}$ & 3 & 1020 & i 100 \\
\hline$m g / L$ & 3 & $<1.0$ & $<1.0$ \\
\hline$m g / h$ & 3 & 226 & 546 \\
\hline $\mathrm{mg} / \mathrm{L}$ & 3 & 0.091 & 0.013 \\
\hline mglt & 3 & 1.38 & 4.62 \\
\hline $\mathrm{mg} / \mathrm{L}$ & 3 & $<1.0$ & 1 \\
\hline$m g / h$ & 3 & 11.3 & 13.8 \\
\hline$m g / L$ & 3 & 11.2 & 12.1 \\
\hline$m g / L$ & 3 & 2.5 & 2.6 \\
\hline $\mathrm{mg} / \mathrm{L}$ & 3 & 57.5 & 59.9 \\
\hline $\mathrm{mg} / \mathrm{h}$ & 3 & 3.8 & 3.8 \\
\hline$m g / h$ & 3 & 0.29 & 0.43 \\
\hline $\mathrm{mg} / \mathrm{L}$ & 3 & $<0.0001$ & 0.00014 \\
\hline$m g / h$ & 3 & $<0.0001$ & $<0.0001$ \\
\hline$m g / L$ & 3 & $<0.001$ & $<0.001$ \\
\hline$m g / L$ & 3 & 0.0033 & 0.094 \\
\hline $\mathrm{mg} / \mathrm{L}$ & 3 & 0.037 & 0.041 \\
\hline$m g /$ & 3 & $<0.05$ & 0.056 \\
\hline$m g / h$ & 3 & 142 & 144 \\
\hline$m g / L$ & 3 & 1.91 & 2.16 \\
\hline $\operatorname{mgn}$ & 3 & 19.1 & 19.5 \\
\hline$m g \Omega$ & 3 & 13.4 & 14.1 \\
\hline $\operatorname{ng} \Omega$ & 3 & 0.585 & 0.586 \\
\hline$n g h$ & 3 & 0.064 & 0.436 \\
\hline$n g /$ & 3 & 2.72 & 2.72 \\
\hline$m g n$ & 3 & 64.3 & 68.9 \\
\hline$n g / L$ & 3 & 0.152 & 0.197 \\
\hline$n g n$ & 3 & $<0.01$ & $<0.01$ \\
\hline$n g / \mathrm{h}$ & 3 & $<0.004$ & $<0.004$ \\
\hline
\end{tabular}

N Modian Maximum if Modian ivaximum

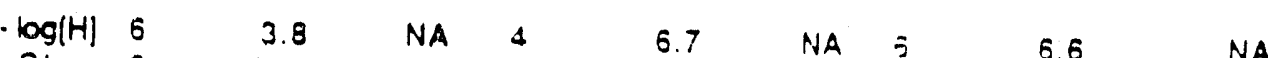

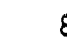

82

73

32
74

5

108

$: 60$

82

63

$11.4 \quad 11.5$

io $10.1=$

$\begin{array}{lll}5.1 & 5.65\end{array}$

$<0.1<0.1$

$<0.0001<0.0001$

$<0.0001<0.0001$

$<0.001<0.001$

$<0.003<0.003$

$<0.0001<0.0001$

$<0.05<0.05$

12.8

$: 3.1$

1.33

2.95

1.38
2.95

0.56

0.56

$<0.05$

$<0.05$

$<0.05$

$<0.05$

0.08

$<0.05$

$<0.05$

$<0.05$

$<0.05$

$<0.01<0.01$

$<0.004<0.004$
3.59

0.003

0.011

13.6

4.9

3.6

1.1

10.1

3.7

0.004

0.033

13.9

5.9

4.2

2.6

10.3

5.3

$<0.1$

$<0.0001$

$<0.0001$

$<0.001$

$<0.003$

0.00028

$<0.05$

16.8

1.33

3.41

0.72

$<0.05$

$<0.05$

0.137

$<0.1$

0.00018

0.00016

$<0.001$

$<0.003$

0.00035

$<0.05$

16.9

1.34

3.43

0.74

$<0.05$

$<0.05$

0.146

0.648

$<0.02$

$<0.01$

$<0.01$

$<0.004$ 
Table 3

Comparison of F1ltered and Unfiltered Metals Concontrations for Four Mile Croek Domstream of F-Area

Concemration Dara From Four Milo Creok Downstream of F Area

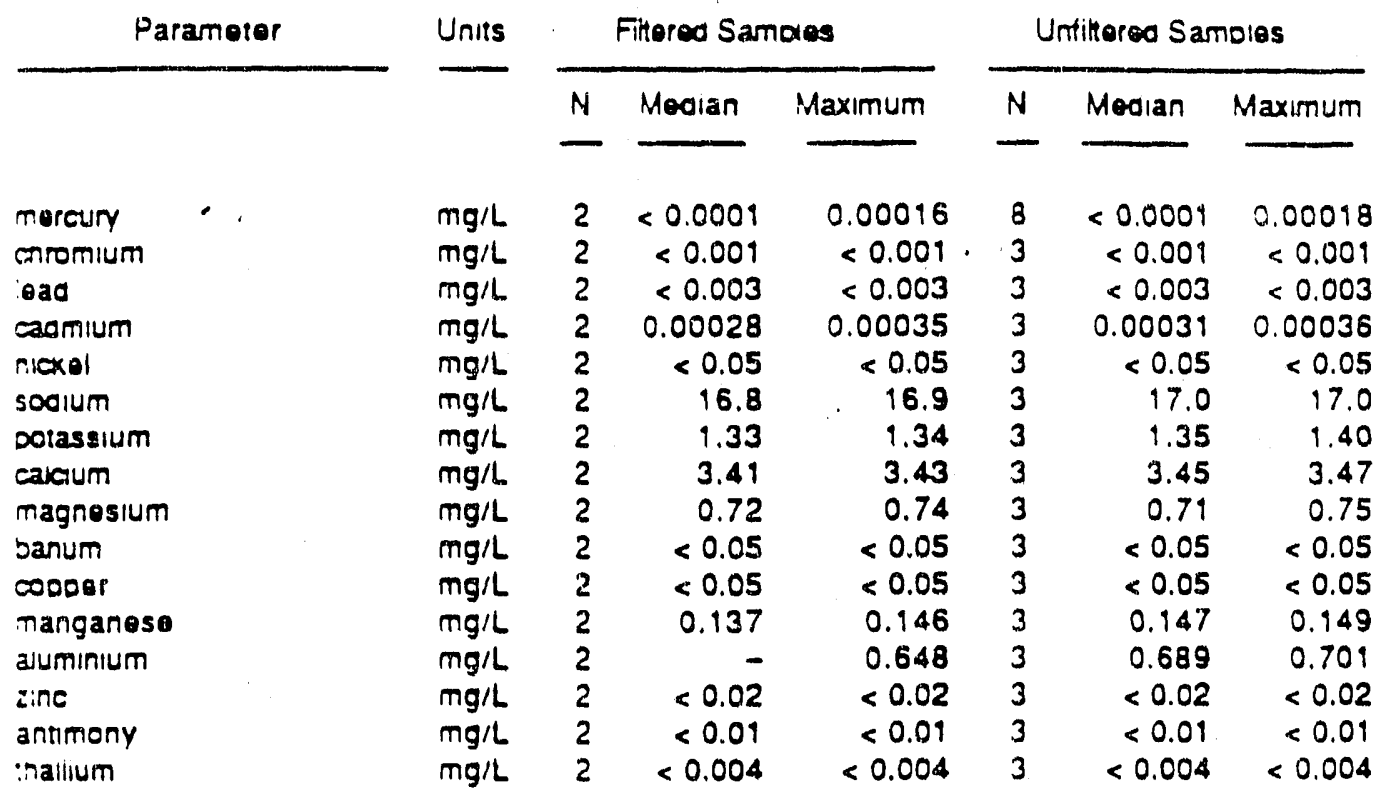




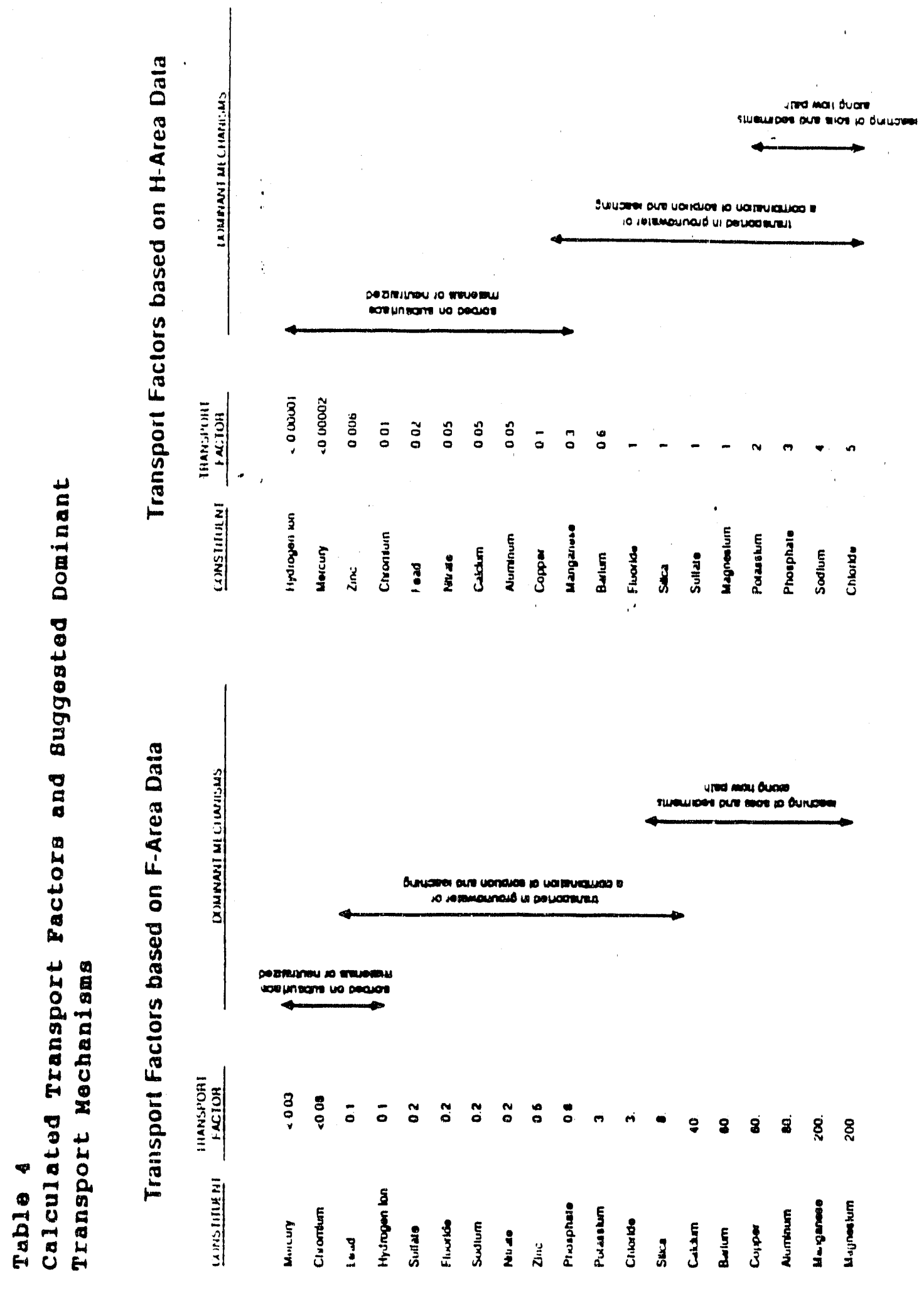




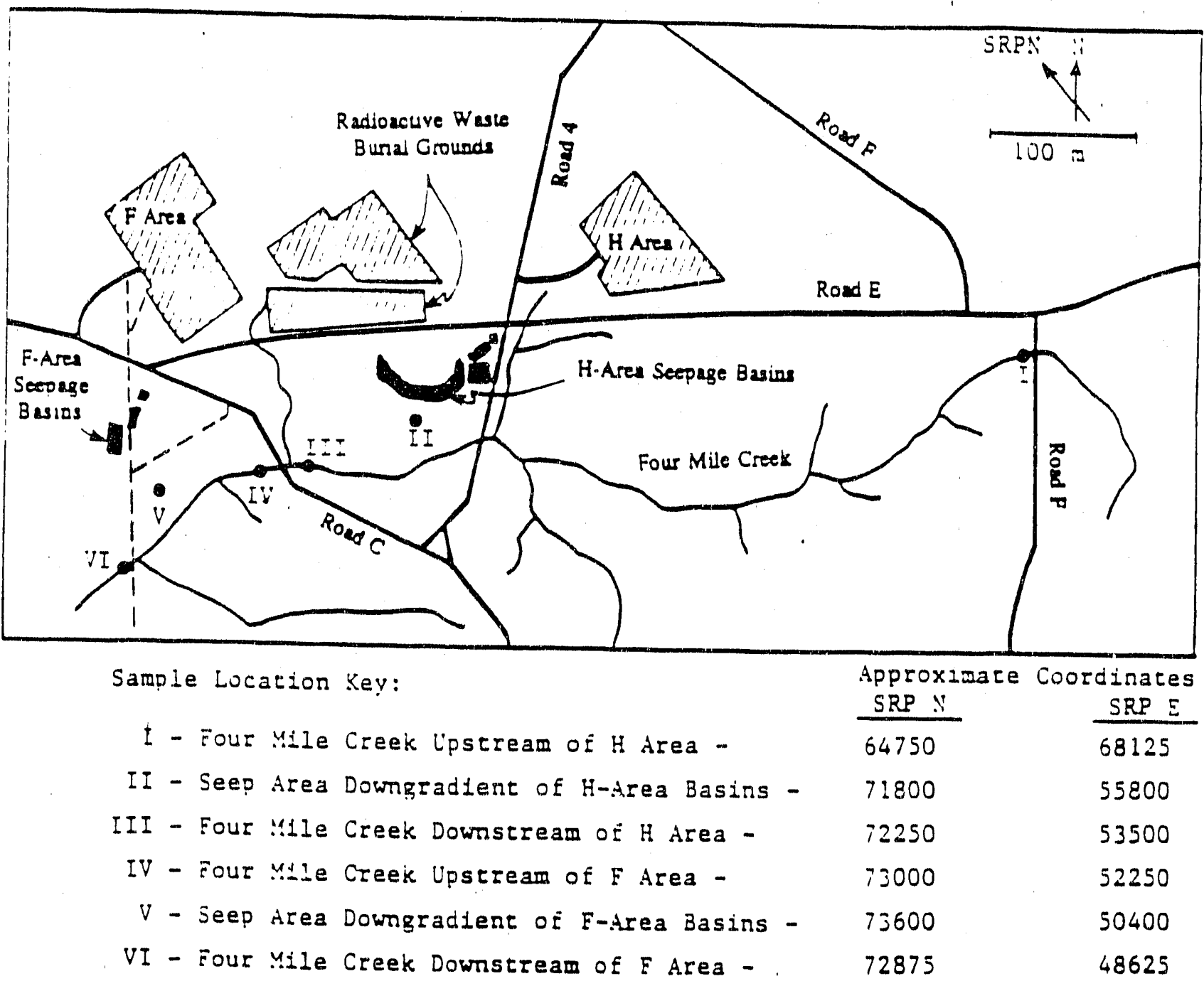

11gure 1. Locationa and Names of tho 8ampling gites 


\section{References}

Anderson, T. J.1980. Methodology for Determination of Envizonmental $129 \mathrm{I}$ and 99 TC. Elfluent and Environmental Radiation 8urvoillance, ASTM STP 698, J. Kelly (ed), Am. Soc. of Testing and Mat., pp 84-94.

Horton, J. H. and G. A. Carothers, 1974. Nitrate in the Separations Area Seepage Basins. DPST-74-293, E. I. du Pont de iemours and Co., Savannah River Laboratory, Aiken SC 29808.

Kantelo, M. V., 1987. Sumary of ${ }^{129}$ I Measurments in Ground and Surface Waters. DPST-87-820, E. I. du Pont de Nemours and Co., Savannah River Laboratory, Aiken SC 29808.

kilizan, T. H., N. L. Kolb, P. Corbo and I. W. Marine, $1987 a$. Environmental Information Document: F-Area Seepage Basins. DPST-85-704, E. I. du Pont de Nemours and Co., Savannah River Laboratory, Aiken SC 29808.

Killian, T. H., N. L. Kolb, P. Corbo and I. W. Marine, 1987b. Envircnmental Information Document: H-Area Seepage Basins. DPST-85-706, E. I. du Pont de Nemours and Co., Savannah River Laboratory, Aiken SC 29808.

Reichert, S. O., 1962. Radionuclides in Groundwater at the Savannah River Plant Waste Disposal Facilities, Jour. Geopbysical Researeb, $67: 4363-4374$.

Reichert, S. 0., 1968. Geology Plays an Important Role in Radioactive Wasre Management. hining Enginaering, September, 1968 . 


\section{Attacbrant 1}

Iist of Parameters Analyzed and Field Notes

(Samples collected 12-08-87) 
Table 1-1

List of Parameters Andyzed

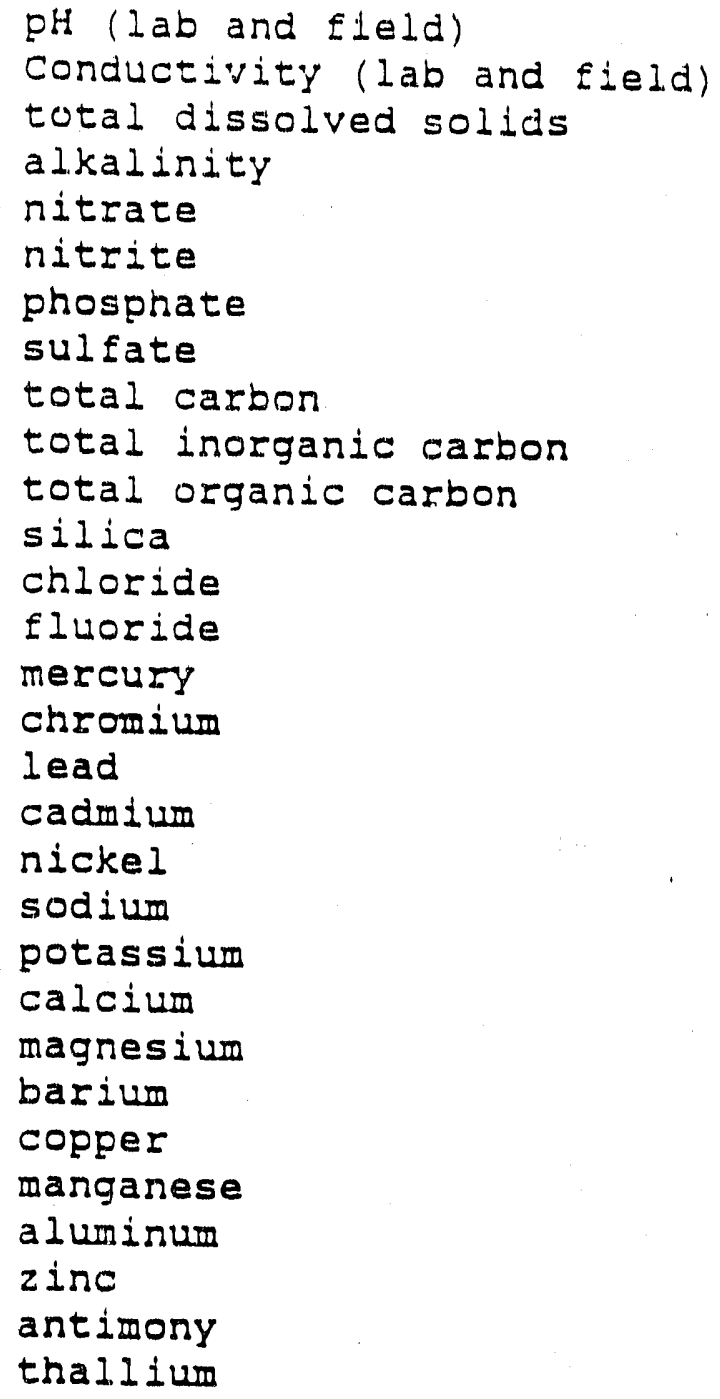

Note: Samples for analysis of a limited number of radionuclides were collected. The results will be reported in a separate report. The samples were collected using clean bottles or beakers. In some cases the amount of solids in the three samples from a site varied significantly. A scheme employing a peristaltic pump is recomended for future sampling in these shallow seep areas that are underlain by flocculant muds. 
Table $1-2$

Fleld Measurements and observationg

H-Area Seepage Basims Samples

Seep Area--Sample numbers FH 002, FH 008, and FH 014

The conductivity of the standing water along the

seep line ranged from $170 \mathrm{us} / \mathrm{cm}$ to $245 \mathrm{us} / \mathrm{cm}$.

The location of the highest conductivity was sampled.

The $\mathrm{pH}$ and conductivity of the actual samples is listed

below.

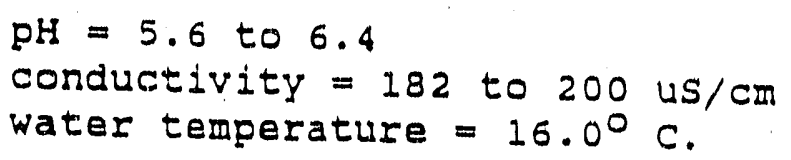

Samples collected along the seep line downgradient of the $H$ Area Seepage Basins--south of Basin H4: The water was turbid containing leaf litter.

H-Area Upstream--Sample numbers FH 003, FH 009, and FH 015

$$
\begin{aligned}
& \mathrm{pH}=6.1 \\
& \text { conductivity }=20 \mathrm{us} / \mathrm{cm} \\
& \text { water temperature }=15.0^{\circ} \mathrm{c} \text {. }
\end{aligned}
$$

Samples collected approximately 50 m downstream of Road $F$ on Four Mile Creek. The stream in this area is small but well defined. Upstream (across the road) is a marshy wetland area. The samples were brown colored and contained some solids.

H-Area Downstream--Sample numbers FH 004, FH 010, and FH 016

$$
\begin{aligned}
& \mathrm{pH}=6.0 \\
& \text { conductivity }=70 \mathrm{us} / \mathrm{cm} \\
& \text { water temperature }=18.0^{\circ} \mathrm{C} \text {. }
\end{aligned}
$$

Samples collected approximately 100 m upstream of Road $C$ on Four Mile creek. The area has a deep clean channel, a high flow and low solids. 
Table 1 - 2 (continued)

Flold Measurements and observationg

E-Area Seepage Basins Samples

Seep Area--Sample numbers FH 001, FH 007, and FH 013

The conductivity of the standing water along the

seep line ranged from 465 us/cm to $1030 \mathrm{us} / \mathrm{cm}$.

The location of the highest conductivity was sampled.

The $\mathrm{pH}$ and conductivity of the actual samples is listed below.

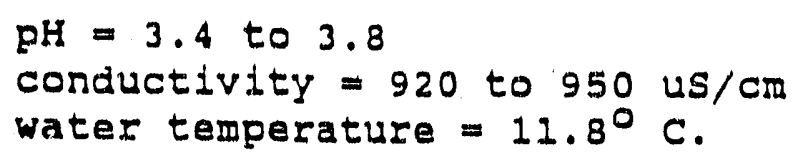

Samples collected along the seep line downgradient of the $F$ Area Seepage Basins--east of well cluster FSB 79. Water was turbid containing leaf litter and a large crop of filamentous algae.

F-Ared Upstream--Sample numbers FH 005,.FH 011, and FH 017

$$
\begin{aligned}
& \mathrm{pH}=6.9 \\
& \text { conductivity }=42 \mathrm{us} / \mathrm{cm} \\
& \text { water temperature }=20.5^{\circ} \mathrm{C} \text {. }
\end{aligned}
$$

Samples collected approximately 100 m downstream of Road $C$ on Four Mile creek. The area is very mucky and marshy and breaks into many small channels in this area. One of the northern channels was selected for sampling. The water was turbid containing flocculant brown mud.

F-Area Downstream--Sample numbers FH 006, FH 018

$$
\begin{aligned}
& \mathrm{pH}=6.3 \\
& \text { conductivity }=70 \mathrm{us} / \mathrm{cm} \\
& \text { water temperature }=17.0^{\circ} \mathrm{C} \text {. }
\end{aligned}
$$

Samples collected approximately 10 m downstream of F-Area steam line road on Four Mile creek. The area has a deep clean channel, a high flow and the least solids of any site. Three additional samples were collected at this site (FH 012, FH 019, and FH 020); the additional samples were collected without filtering the metals subsample. 
Attacbuent 2

Complate Data set 


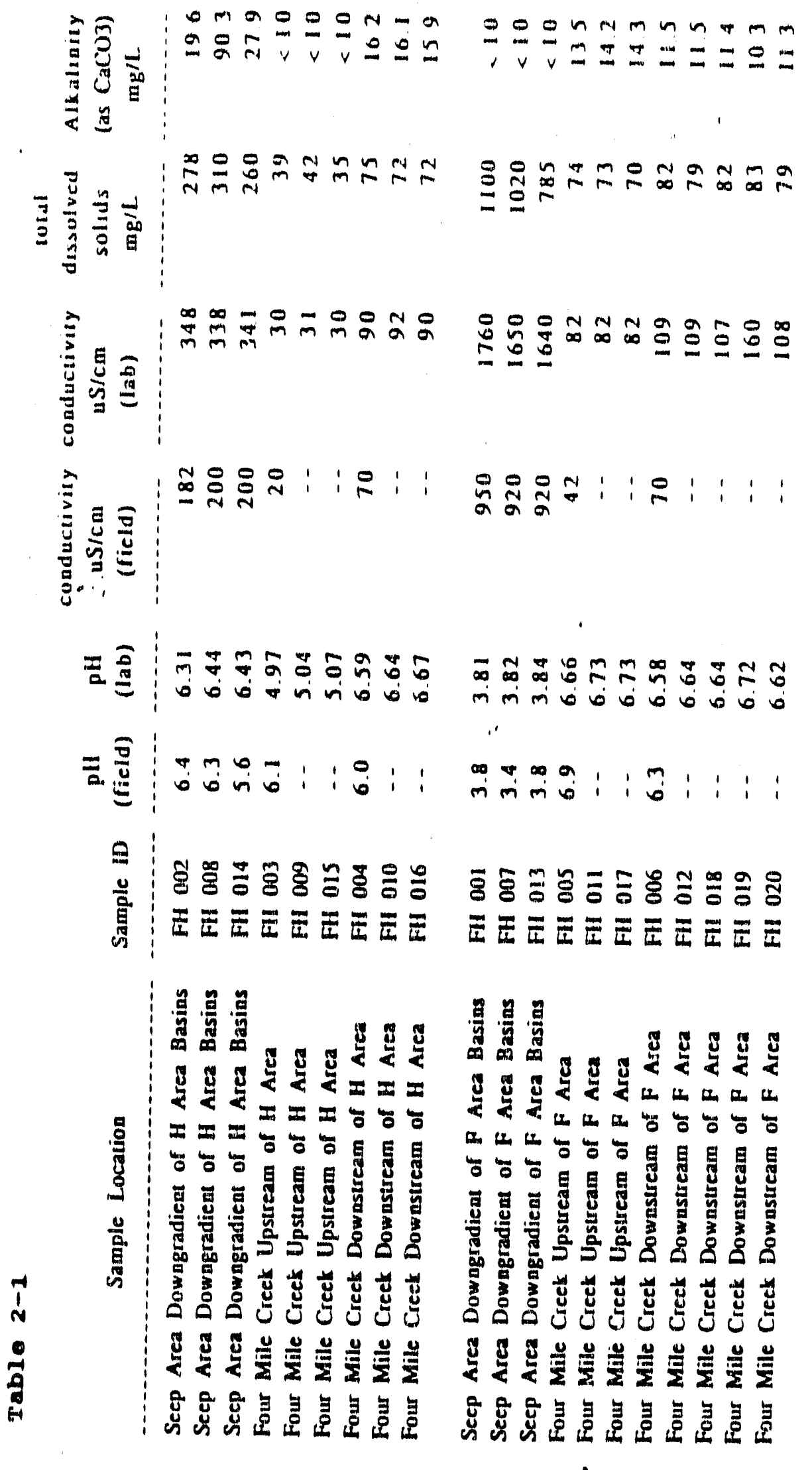




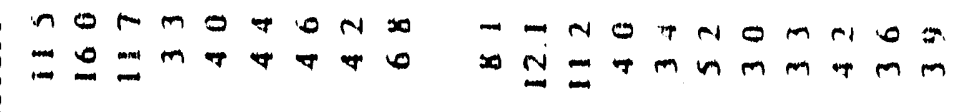

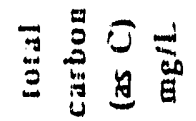

n $\infty$ a a m m a m

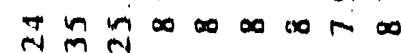

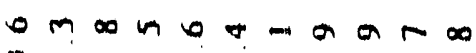

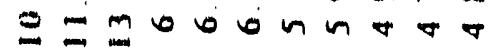

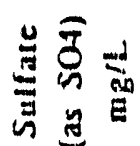

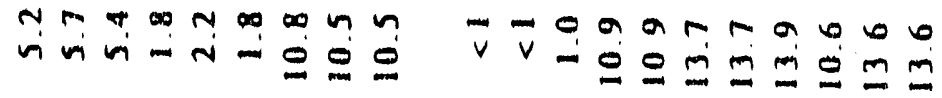

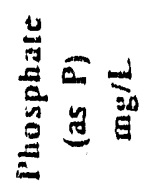

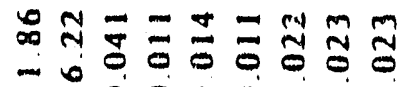

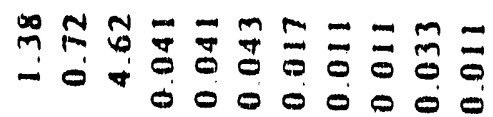

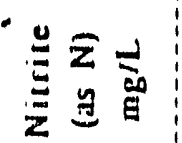

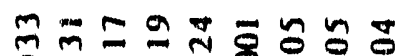

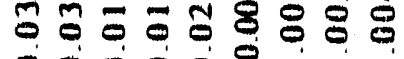

o

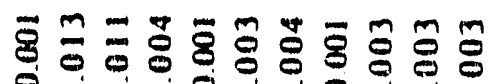

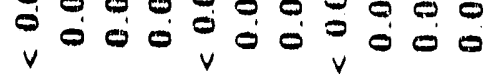

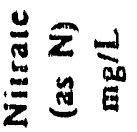

ตำ

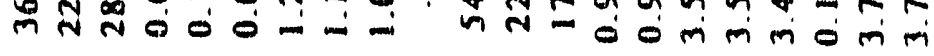

$\cong$ 音

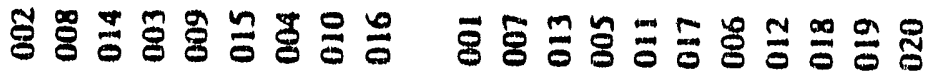

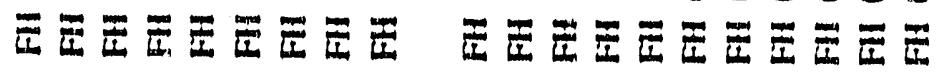




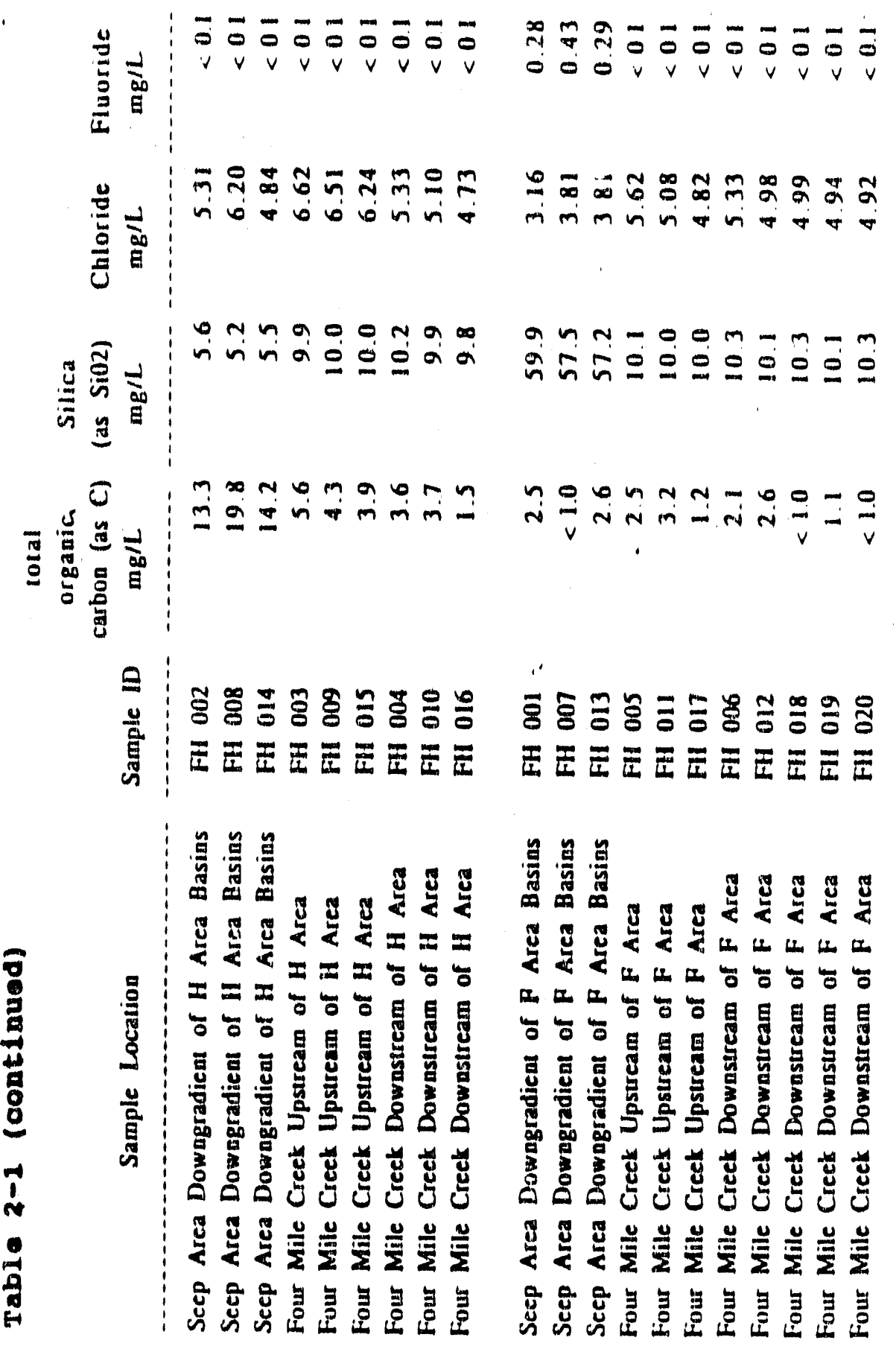




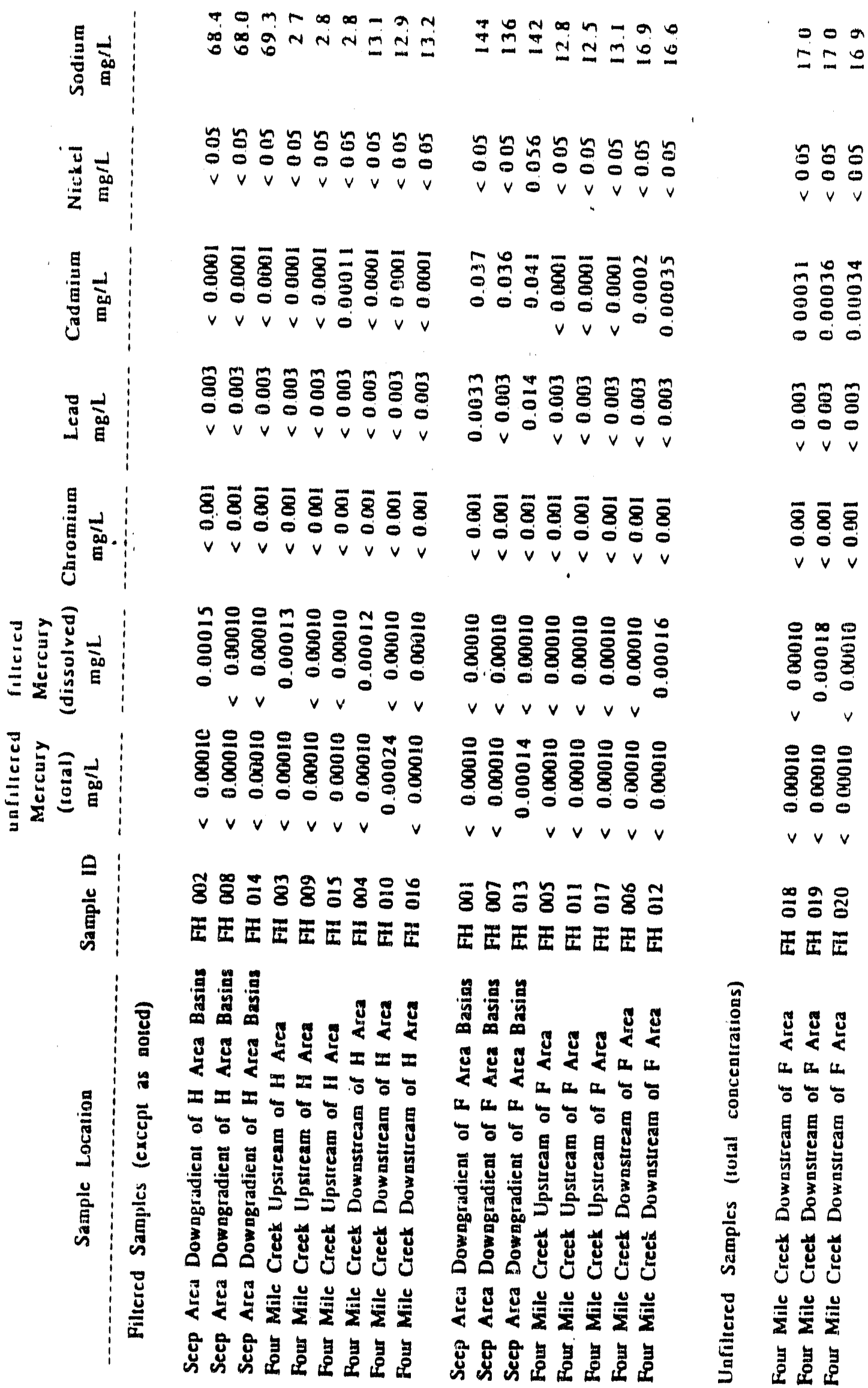




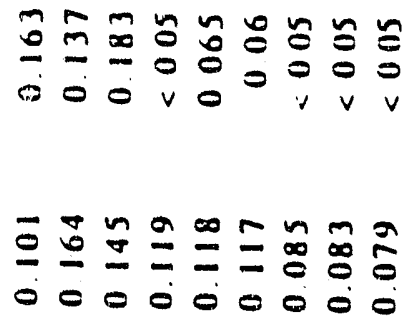

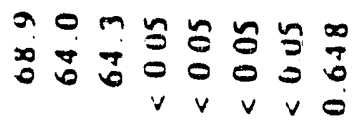

$\cong \underset{0}{\infty} \overline{0}$

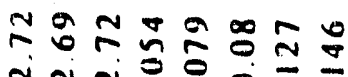

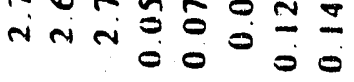

$\hat{n} \mathfrak{z}$

$0=$

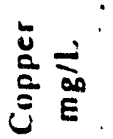

nूw nn nn

0
$v$

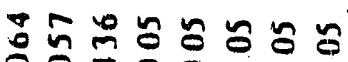

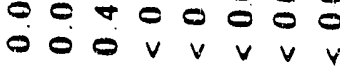

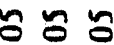

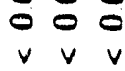

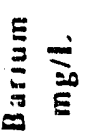

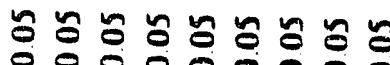

000000000

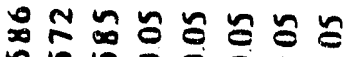

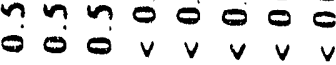

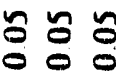

$v v v$

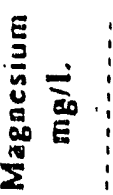

N

- - - 00000

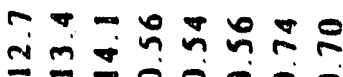

$\approx a \overline{1}$

o 00

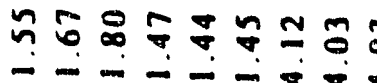

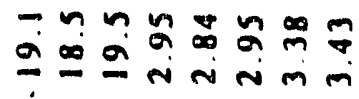

요

inim

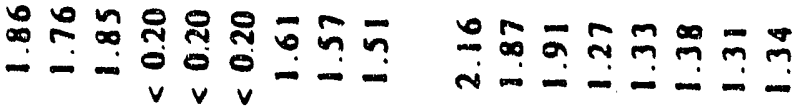

กิก ำ

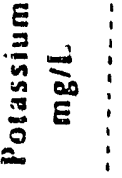

$-\overline{-}$

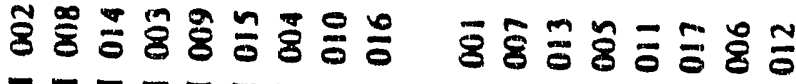

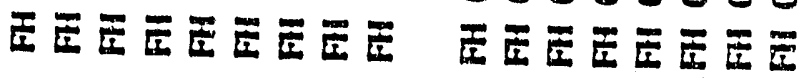

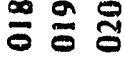

医正正

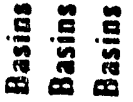

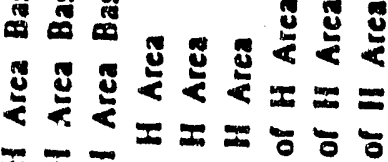

$\Sigma=ニ \div \div \div$

ㄴํำ

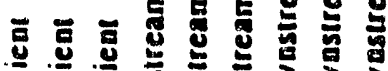

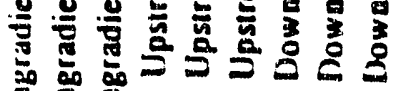

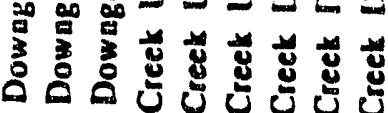

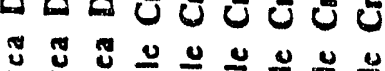

兽总兽

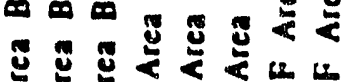

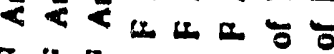

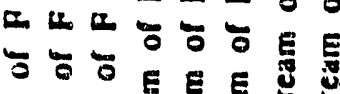

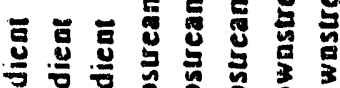

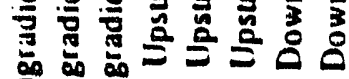

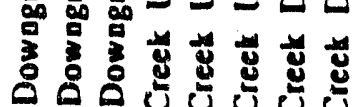

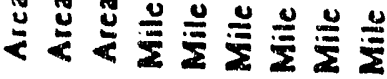

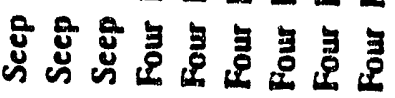

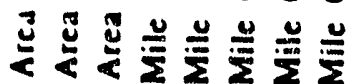

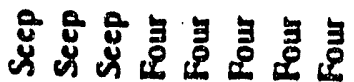




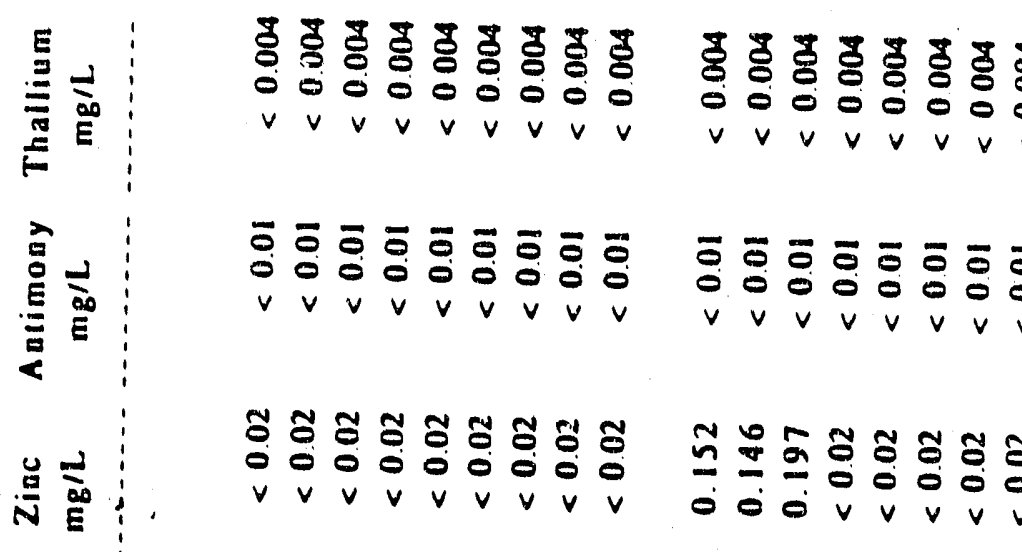

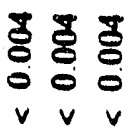

$\begin{array}{lll}\bar{\sigma} & \overline{0} & \bar{\sigma} \\ 0 & 0 \\ v & v \\ v & v\end{array}$

ธีธ รี o 00

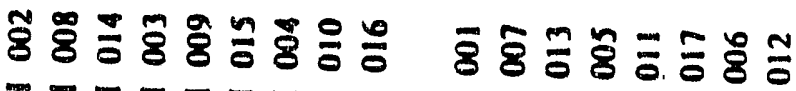

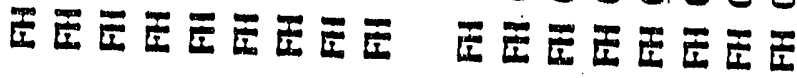

正웡 융 考实

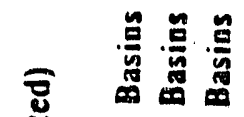




\section{Appendix B}

Sample
HSP-94
HSP -95
HSP -96
HSP-97
HSP-98
HSP -99
HSP-100
HSP-101
HSP-102
HSP-103
HSP -104
HSP-105
HSP -106
HSP-107
HSP -108
HSP-109

Tritium conc. (pci/ml)

$\begin{array}{llr}(3 / 89) & (1 / 90) & \mathrm{d}(1 / 90- \\ 240 & 750 & +510 \\ 570 & 750 & +180 \\ 1100 & 1600 & +500 \\ 1100 & 1500 & +400 \\ 1800 & 1500 & -300 \\ 1200 & 1200 & 0 \\ 1300 & 1800 & +500 \\ 690 & 950 & +260 \\ 700 & 610 & -90 \\ 510 & 400 & -110 \\ 660 & 810 & +150 \\ 740 & 810 & +70 \\ 640 & 740 & +100 \\ 480 & 460 & -20 \\ 680 & 910 & +230 \\ 680 & 230 & -450 \\ 818 & 939 & +121\end{array}$

$$
s_{d}^{2}=\frac{510^{2}+180^{2}+500^{2}+\ldots+450^{2}}{16}=8900
$$

$$
\begin{gathered}
s_{d}-\sqrt{s_{d}^{2}}-298 \\
H_{0}: d=0 . \\
t_{0}=\frac{\bar{d}-0}{s_{d}}=\frac{121}{298}=0.40
\end{gathered}
$$

$\operatorname{Pr}(t>0.40) \approx 0.35$ 
Appendix C

\begin{tabular}{|c|c|c|c|c|c|c|}
\hline $\begin{array}{l}\text { station } \\
\text { Designati }\end{array}$ & $\begin{array}{l}\text { Cond. } \\
(\mu \mathrm{mhos} / \mathrm{cm})\end{array}$ & $\mathrm{pH}$ & & $\begin{array}{l}\text { Temp } \\
\left(\begin{array}{cc}0 & \mathrm{c} .\end{array}\right)\end{array}$ & \multicolumn{2}{|c|}{$\begin{array}{l}\text { Sampling } \\
\text { Date }\end{array}$} \\
\hline HSP-0I & 156 & 5 & & 10 & & $03 / 02 / 89$ \\
\hline $\mathrm{HSP}-02$ & 256 & 5.2 & & 11 & 2 & $03 / 02 / 89$ \\
\hline HSP-03 & 468 & 5.2 & & 12 & 3 & $03 / 02 / 89$ \\
\hline $\mathrm{HSP}-04$ & 292 & 5.8 & & 10 & 5 & $03 / 02 / 89$ \\
\hline HSP-0 5 & 281 & 6.2 & & 10 & 4 & $03 / 02 / 89$ \\
\hline HSP-06 & 318 & 6.1 & & 10 & 5 & $03 / 02 / 89$ \\
\hline HSP -07 & dry hole dry & hole & dry & hole & dry hole & $03 / 02 / 89$ \\
\hline HSP-08 & 556 & 5.7 & & 11 & 4 & $03 / 02 / 89$ \\
\hline HSP-09 & 317 & 5.5 & & 11 & 4 & $03 / 02 / 89$ \\
\hline HSP -10 & 171 & 5.6 & & 10 & 6 & $03 / 02 / 89$ \\
\hline HSP- 11 & 80 & 5.1 & & 11 & 4 & $03 / 02 / 89$ \\
\hline HSP -12 & 218 & 5.2 & & 11. & 2 & $03 / 02 / 89$ \\
\hline HSP -13 & 592 & 6.2 & & 11 & 3 & $03 / 03 / 89$ \\
\hline$H S P-14$ & 393 & 6.2 & & 11 & surface & $03 / 03 / 89$ \\
\hline HSP -15 & 82 & 5.2 & & 11 & surface & $03 / 03 / 89$ \\
\hline HSP -16 & 215 & 5 & & 11 & 4 & $03 / 03 / 89$ \\
\hline HSP -17 & dry hole dry & hole & $d r y$ & hole & dry hole & $03 / 03 / 89$ \\
\hline HSP -18 & 149 & 5.5 & & 11 & 1 & $03 / 03 / 89$ \\
\hline HSP -19 & 148 & $4 \cdot 7$ & & 12 & 4 & $03 / 03 / 89$ \\
\hline $\mathrm{HSP}-2 \mathrm{O}$ & 183 & 6.2 & & 15 & surface & $03 / 03 / 89$ \\
\hline $\mathrm{HSP}-21$ & 189 & 6.1 & & 14 & 1 & $03 / 06 / 89$ \\
\hline HSP -22 & 161 & 5.4 & & 15 & 1 & $03 / 06 / 89$ \\
\hline HSP -23 & 184 & 4.7 & & 15 & 4 & $03 / 06 / 89$ \\
\hline HSP-24 & 140 & 5.2 & & 14 & 3 & $03 / 06 / 89$ \\
\hline HSP -25 & 135 & 4.7 & & 15 & 7 & $03 / 06 / 89$ \\
\hline HSP -26 & 205 & 5.2 & & 14 & 2 & $03 / 06 / 89$ \\
\hline HSP -27 & 202 & 5.4 & & 14 & 6 & $03 / 06 / 89$ \\
\hline HSP -28 & 244 & 5.7 & & 14 & 2 & $03 / 06 / 89$ \\
\hline HSP-29 & 257 & 5.2 & & 15 & 3 & $03 / 06 / 89$ \\
\hline HSP -30 & 221 & 5.3 & & 15 & 3 & $03 / 06 / 89$ \\
\hline HSP -31 & 214 & 5.6 & & 14 & 1 & $03 / 06 / 89$ \\
\hline HSP- 32 & 215 & 5.5 & & 14 & 6 & $03 / 06 / 89$ \\
\hline HSP -33 & 176 & 5.8 & & 14 & 6 & $03 / 06 / 89$ \\
\hline HSP -34 & 331 & 5.8 & & 14 & 8 & $03 / 06 / 89$ \\
\hline HSP -35 & 121 & 5.8 & & 13 & 1 & $03 / 07 / 89$ \\
\hline HSP -36 & 210 & 5.4 & & 13 & 7 & $03 / 07 / 89$ \\
\hline HSP -37 & 231 & 5.3 & & 13 & 10 & $03 / 07 / 89$ \\
\hline HSP -38 & 227 & 4.9 & & 12 & 5 & $03 / 07 / 89$ \\
\hline HSP -39 & 242 & 5.1 & & 12.5 & 4 & $03 / 07 / 89$ \\
\hline HSP -40 & 256 & 4.7 & & 12 & 3 & $03 / 07 / 89$ \\
\hline HSP -41 & 224 & 4.8 & & 12 & 8 & $03 / 07 / 89$ \\
\hline HSP -42 & 310 & 5.2 & & 12 & 8 & $03 / 07 / 89$ \\
\hline HSP -43 & 413 & 5.3 & & 12 & 9 & $03 / 07 / 89$ \\
\hline $\mathrm{HSP}-44$ & 333 & 5.2 & & 12 & 8 & $03 / 07 / 89$ \\
\hline HSP -45 & 255 & 4.5 & & 12 & 6 & $03 / 07 / 89$ \\
\hline HSP -46 & 318 & 5.5 & & 12 & 8 & $03 / 07 / 89$ \\
\hline HSP -47 & 426 & 5.4 & & 12 & 6 & $03 / 07 / 89$ \\
\hline HSP -48 & 569 & 4.5 & & 12 & 10 & $03 / 07 / 89$ \\
\hline HSP -49 & 551 & 4.4 & & 13 & 8 & $03 / 07 / 89$ \\
\hline
\end{tabular}


Appendix C

WSRC-RP-90-0591

\begin{tabular}{|c|c|c|c|c|c|c|c|c|}
\hline $\mathrm{HSP}-5 \mathrm{O}$ & & 537 & & 4.4 & & 12 & 7 & $03 / 07 / 8$ \\
\hline HSP-51 & & 654 & & 4.8 & & 12 & 6 & $03 / 07 / 8$ \\
\hline HSP -52 & & 699 & & 4.1 & & 12 & 7 & $03 / 07 / 8$ \\
\hline HSP -53 & & 669 & & 4.4 & & 12 & 10 & $03 / 07 / 8$ \\
\hline HSP-54 & & 631 & & 4.4 & & 12 & 8 & $03 / 07 / 8$ \\
\hline HSP -55 & & 56.1 & & 6.9 & & 10 & 6 & $03 / 09 / 8$ \\
\hline HSP -56 & & 452 & & 5.9 & & 11 & 5 & $03 / 09 / 8$ \\
\hline HSP -57 & & 581 & & 5.5 & & 12 & 5 & $03 / 09 / 8$ \\
\hline HSP -58 & & 551 & & 6 & & 11 & 4 & $03 / 09 / \varepsilon$ \\
\hline HSP -59 & & 280 & & 6.1 & & 11 & 8 & $03 / 09 / 8$ \\
\hline HSP -60 & & 473 & & 5.9 & & 11 & 8 & $03 / 09 / 8$ \\
\hline HSP -61 & & 85 & & 5.9 & & 11 & 8 & $03 / 09 / 8$ \\
\hline HSP -62 & & 155 & & 5.8 & & 11 & 7 & $03 / 09 / 8$ \\
\hline HSP -63 & & 34 & & 5.1 & & 10 & 3 & $03 / 09 / 8$ \\
\hline HSP -64 & & 38 & & 4.7 & & 10 & 4 & $03 / 09 / 8$ \\
\hline HSP -65 & & 51 & & 5.1 & & 11 & 4 & $03 / 09 / 8$ \\
\hline HSP -66 & & 49 & & 4.6 & & 12 & 3 & $03 / 09 / 8$ \\
\hline HSP -67 & & 31 & & 5 & & 10 & 8 & $03 / 09 / 8$ \\
\hline HSP -68 & & 39 & & 4.9 & & 10 & 3 & $03 / 09 / 8$ \\
\hline HSP -69 & & 35 & & 4.4 & & 10 & 1 & $03 / 09 / 8$ \\
\hline HSP -70 & & 30 & & 4.6 & & 10 & 8 & $03 / 09 / 8$ \\
\hline HSP -71 & & 40 & & 5.1 & & 9 & 3 & $03 / 09 / 8$ \\
\hline HSP -72 & $d r y$ & hole & dry & hole & $d r y$ & hole & dry hole & $03 / 09 / 8$ \\
\hline HSP -73 & dry & hole & dry & hole & dry & hole & dry hole & $03 / 09 / 8$ \\
\hline HSP -74 & & 73 & & 6 & & 9 & 4 & $03 / 10 / 8$ \\
\hline HSP -75 & & 81 & & 3.8 & & 11 & 5 & $03 / 10 / 8$ \\
\hline HSP -76 & & 146 & & 5.7 & & 11 & 7 & $03 / 10 / 8$ \\
\hline HSP -77 & & 43 & & 5 & & 11 & 4 & $03 / 10 / 8$ \\
\hline HSP -78 & & 53 & & 4.6 & & 12 & surface & $03 / 10 / 8$ \\
\hline HSP -79 & & 80 & & 5.3 & & 13 & 7 & $03 / 10 / 8$ \\
\hline HSP -80 & & 76 & & 5 & & 13 & 5 & $03 / 10 / 8$ \\
\hline HSP-81 & & 39 & & 5.2 & & 13 & 6 & $03 / 10 / 8$ \\
\hline HSBP -82 & & 60 & & 4.9 & & 13 & 5 & $03 / 10 / 8$ \\
\hline HSBP -83 & & 187 & & 5.5 & & 11 & 1 & $03 / 10 / 89$ \\
\hline HSP -84 & & 51 & & 4,9 & & 12 & surface & $03 / 10 / 8$ \\
\hline HSP -85 & & 22 & & 4.5 & & 11 & 4 & $03 / 10 / 8$ \\
\hline HSP -86 & & 41 & & 4.7 & & 12 & 9 & $03 / 20 / 8$ \\
\hline HSP -87 & & 38 & & 4 & & 12 & 5 & $03 / 10 / 8$ \\
\hline HSP -88 & & 49 & & 4.3 & & 11 & surface & $03 / 10 / 8$ \\
\hline HSP-89 & & 56 & & 4.7 & & 12 & 8 & $03 / 13 / 8$ \\
\hline HSP -90 & & 59 & & 4.4 & & 12 & 7 & $03 / 13 / 8$ \\
\hline HSP -91 & & 29 & & 5.2 & & 12 & 1 & $03 / 13 / 8$ \\
\hline HSP -92 & & 26 & & 4.4 & & 12 & surface & $03 / 13 / 8$ \\
\hline HSP -93 & & 48 & & 4.1 & & 12 & 4 & $03 / 13 / 8$ \\
\hline HSP-94 & & 50 & & 4.2 & & 12 & 8 & $03 / 13 / 8$ \\
\hline HSP-95 & & 32 & & 4.5 & & 12 & 3 & $03 / 13 / 8$ \\
\hline HSP -96 & & 54 & & 4.1 & & 12 & 8 & $03 / 13 / 8$ \\
\hline HSP-97 & & 37 & & 4.3 & & 12 & 8 & $03 / 13 / 8$ \\
\hline $5 P-98$ & & 34 & & 4.7 & & 12 & 10 & $03 / 13 / 8$ \\
\hline P-99 & & 55 & & 4.7 & & 12 & 2 & $03 / 13 / 8$ \\
\hline $3 P-100$ & & 44 & & 4.7 & & 13 & 3 & $03 / 13 / 8$ \\
\hline HSP-101 & & 37 & & 4.8 & & 13.5 & 8 & $03 / 13 / 8$ \\
\hline
\end{tabular}


Appendix C

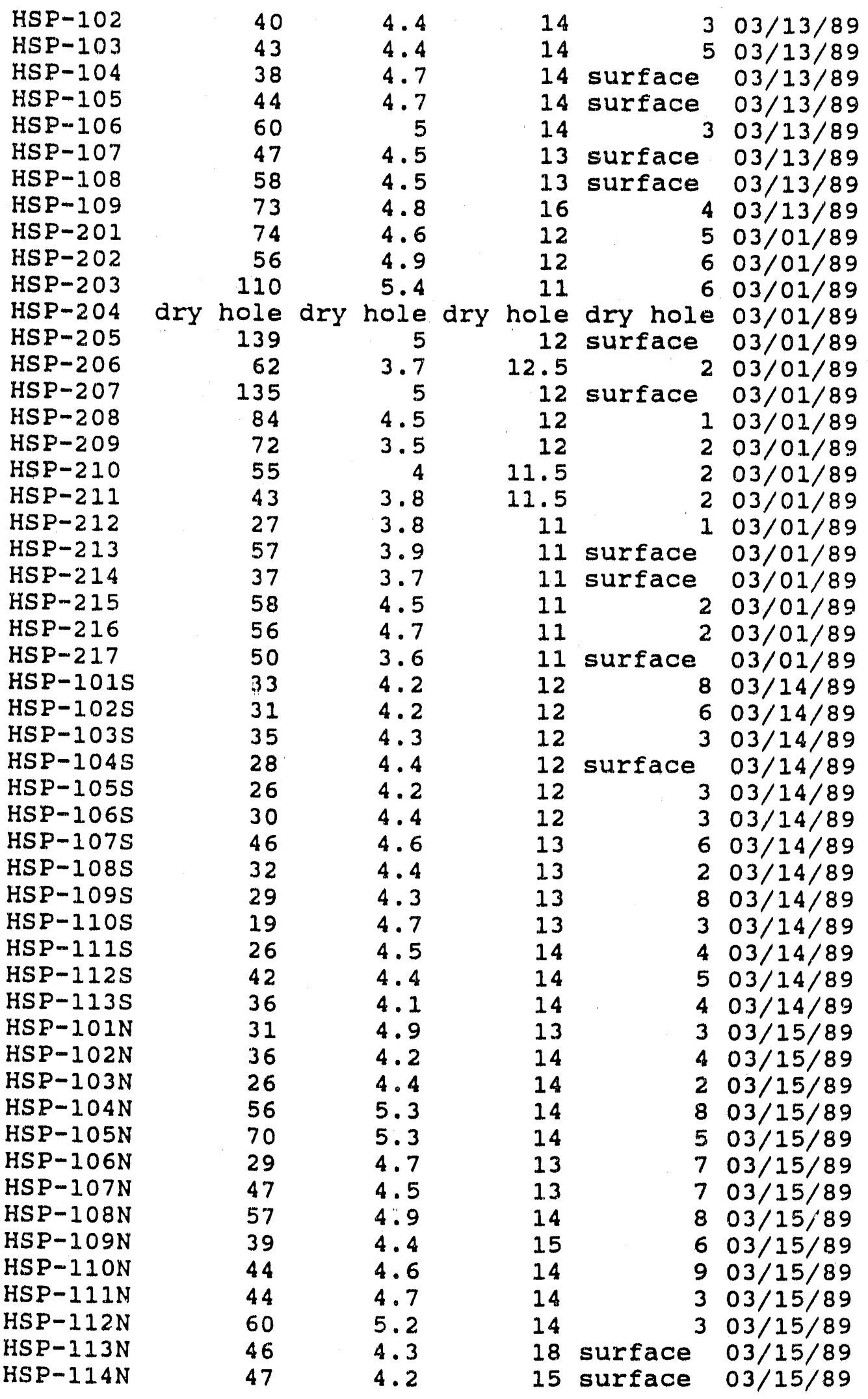


Appendix $\mathrm{C}$

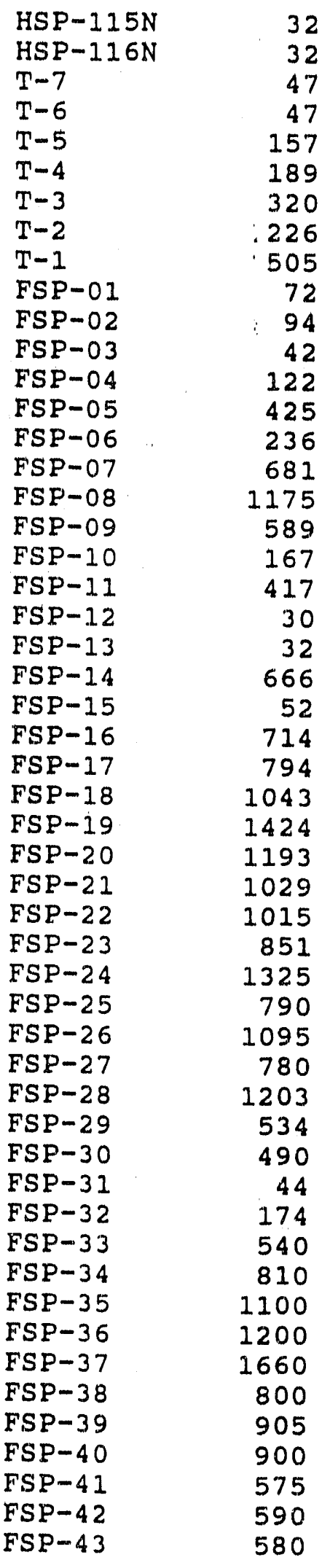

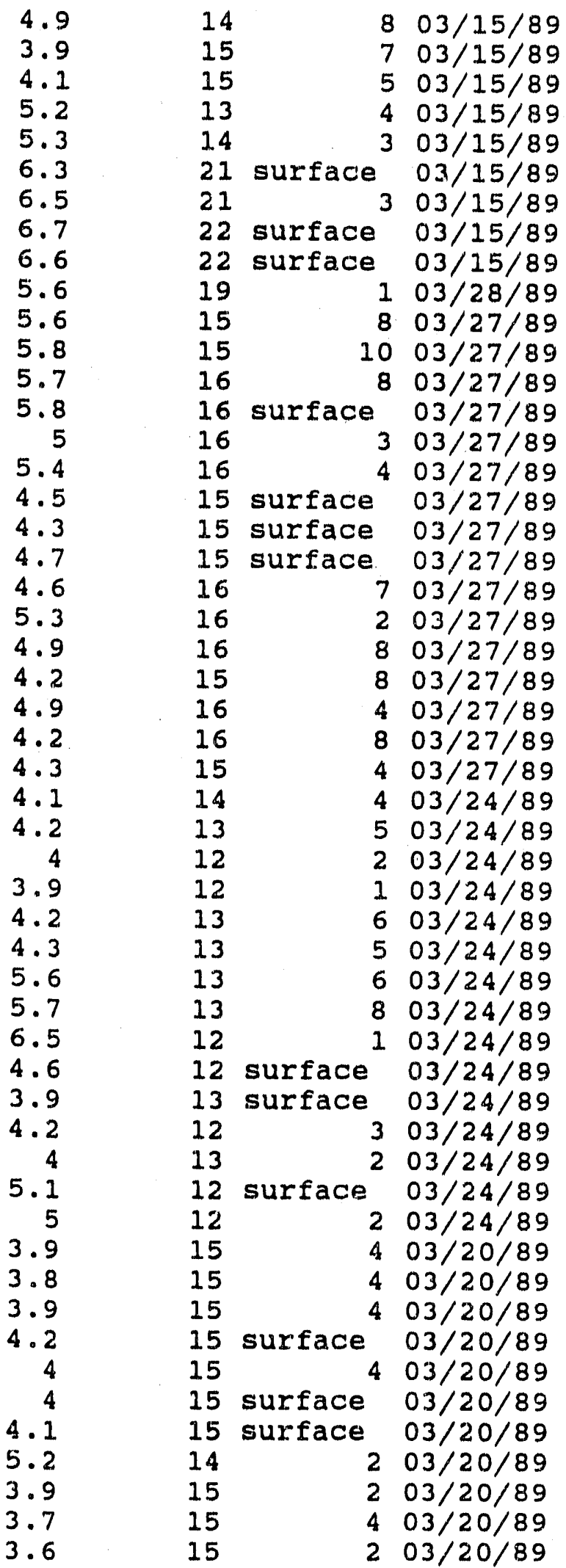


Appendix C

\begin{tabular}{|c|c|c|c|c|c|}
\hline $\begin{array}{l}\text { FSP }-44 \\
\text { FSP }-43\end{array}$ & 469 & 4.7 & 15 & 2 & $03 / 20 / 89$ \\
\hline FSP- -44 & 229 & 5.2 & 15 & 5 & $\begin{array}{l}03 / 17 / 89 \\
03 / 17 / 89\end{array}$ \\
\hline$F S P-45$ & 96 & $\begin{array}{l}2.2 \\
5.3\end{array}$ & $\begin{array}{l}14 \\
15\end{array}$ & $\begin{array}{r}10 \\
8\end{array}$ & $\begin{array}{l}03 / 17 / 89 \\
03 / 17 / 89\end{array}$ \\
\hline FSP -46 & 40 & 4.7 & 15 & $\begin{array}{l}8 \\
4\end{array}$ & $03 / 17 / 89$ \\
\hline FSP -47 & 52 & 4.7 & 15 & 6 & $03 / 17 / 89$ \\
\hline FSP -48 & 92 & 5.2 & 15 & 4 & $03 / 17 / 89$ \\
\hline FSP-49 & 143 & 5 & 15 & 8 & $03 / 17 / 89$ \\
\hline FSP-50 & 60 & 4.7 & 14 & 7 & $03 / 17 / 89$ \\
\hline FSP-51 & 30 & 4.9 & 15 & 5 & $03 / 17 / 89$ \\
\hline FSP-52 & 18 & 4.5 & 15 & 2 & $03 / 17 / 89$ \\
\hline F'SP-53 & 60 & 4.4 & 15 & surface & $03 / 17 / 89$ \\
\hline FSP- 54 & 30 & 4.3 & 15 & 1 & $03 / 17 / 89$ \\
\hline FSP -55 & 50 & 4.6 & 14 & 8 & $03 / 17 / 89$ \\
\hline FSP-56 & 34 & 4.5 & 14 & 8 & $03 / 17 / 89$ \\
\hline FSP-57 & 31 & 4.6 & 15 & 8 & $03 / 17 / 89$ \\
\hline FSP-58 & 66 & 4.3 & 15 & 4 & $03 / 17 / 89$ \\
\hline FSP-59 & 29 & 4.9 & 15 & 5 & $03 / 17 / 89$ \\
\hline FSP-200 & 315 & 5.6 & 16 & surface & $03 / 28 / 89$ \\
\hline FSP-201 & 305 & 5.4 & 15 & 6 & $03 / 28 / 89$ \\
\hline FSP-202 & 446 & 4.8 & 17 & surface & $03 / 28 / 89$ \\
\hline $\mathrm{FSP}-203$ & 308 & 5.6 & 15 & surface & $03 / 28 / 89$ \\
\hline FSP-204 & 895 & 4.4 & 15 & surface & $03 / 28 / 89$ \\
\hline FSP-205 & 1233 & 4 & 15 & surface & $03 / 28 / 89$ \\
\hline FSP-206 & 1036 & 4 & 15 & 6 & $03 / 28 / 89$ \\
\hline FSP-207 & 445 & 4.1 & 16 & 2 & $03 / 28 / 89$ \\
\hline FSP-208 & 41 & 5.5 & 16 & 7 & $03 / 28 / 89$ \\
\hline FSP-209 & 199 & 5 & 18 & 2 & $03 / 28 / 89$ \\
\hline FSP-210 & 53 & 5.5 & 15 & 12 & $03 / 28 / 89$ \\
\hline FSP-211 & 40 & 5 & 16 & 8 & $03 / 28 / 89$ \\
\hline FSP-212 & 193 & 5.6 & 15 & 6 & $03 / 28 / 89$ \\
\hline FSP-213 & 860 & 4.6 & 15 & 8 & $03 / 28 / 89$ \\
\hline$F S F-214$ & 1032 & 4.2 & 15 & 4 & $03 / 28 / 89$ \\
\hline FSP-215 & 739 & 4.1 & 15 & 6 & $03 / 28 / 89$ \\
\hline FSP-216 & 854 & 4 & 15 & 12 & $03 / 28 / 89$ \\
\hline FSP-2 17 & 938 & 4.1 & 16 & surface & $03 / 28 / 89$ \\
\hline FSP-218 & 401 & 4.6 & 16 & 8 & $03 / 29 / 89$ \\
\hline FSP-219 & 426 & 5.4 & 16 & 6 & $03 / 29 / 89$ \\
\hline FSP -220 & 147 & 4.8 & 16 & 9 & $03 / 29 / 89$ \\
\hline FSP-2 21 & 140 & 5.8 & 16 & surface & $03 / 29 / 89$ \\
\hline FSP -222 & 123 & 6.3 & 16 & surface & $03 / 29 / 89$ \\
\hline$F S P-223$ & 115 & 6 & 16 & 14 & $03 / 29 / 89$ \\
\hline FSP-224 & 112 & 5.3 & 16 & 10 & $03 / 29 / 89$ \\
\hline FSP-225 & 254 & 5 & 16 & 1 & $03 / 29 / 89$ \\
\hline FSP-226 & 306 & 5.1 & 17 & 3 & $03 / 29 / 89$ \\
\hline FSP-227 & 204 & 5.1 & 17 & 3 & $03 / 29 / 89$ \\
\hline FSP-228 & 259 & 5.2 & 17 & 3 & $03 / 29 / 89$ \\
\hline FSP-229 & 54 & 4.8 & 16 & 5 & $03 / 29 / 89$ \\
\hline FSP-230 & 95 & 4.5 & 16 & 5 & $03 / 29 / 89$ \\
\hline FSP-231 & 70 & 4.3 & 16 & surface & $03 / 29 / 89$ \\
\hline FSP-232 & 96 & 5.7 & 16 & surface & $03 / 29 / 89$ \\
\hline$F S P-2.33$ & 103 & 5.5 & 16 & 4 & $03 / 29 / 89$ \\
\hline
\end{tabular}

$$
\begin{array}{rrr}
5 & 2 & 03 / 20 / 89 \\
5 & 5 & 03 / 17 / 89 \\
4 & 10 & 03 / 17 / 89 \\
5 & 8 & 03 / 17 / 89 \\
5 & 4 & 03 / 17 / 89 \\
5 & 6 & 03 / 17 / 89 \\
5 & 4 & 03 / 17 / 89 \\
5 & 8 & 03 / 17 / 89 \\
4 & 7 & 03 / 17 / 89 \\
5 & 5 & 03 / 17 / 89 \\
5 & 2 & 03 / 17 / 89 \\
5 & \text { surface } & 03 / 17 / 89 \\
5 & 1 & 03 / 17 / 89 \\
4 & 8 & 03 / 17 / 89 \\
4 & 8 & 03 / 17 / 89 \\
5 & 8 & 03 / 17 / 89 \\
5 & 4 & 03 / 17 / 89 \\
5 & 5 & 03 / 17 / 89 \\
6 & \text { surface } & 03 / 28 / 89 \\
5 & 6 & 03 / 28 / 89 \\
7 & \text { surface } & 03 / 28 / 89 \\
5 & \text { surface } & 03 / 28 / 89 \\
5 & \text { surface } & 03 / 28 / 89 \\
5 & \text { surface } & 03 / 28 / 89 \\
5 & 6 & 03 / 28 / 89 \\
6 & 2 & 03 / 28 / 89 \\
6 & 7 & 03 / 28 / 89 \\
6 & 2 & 03 / 28 / 89 \\
8 & 12 & 03 / 28 / 89 \\
5 & 8 & 03 / 28 / 89 \\
6 & 6 & 03 / 28 / 89 \\
5 & 8 & 03 / 28 / 89 \\
5 & 4 & 03 / 28 / 89 \\
5 & 6 & 03 / 28 / 89 \\
5 & 12 & 03 / 28 / 89 \\
5 & 5 & 03 / 29 / 89
\end{array}
$$

WSRC-RP-90-0591 
Appendix $\mathrm{C}$

FSP-234

FSP -235

FSP -236

FSP -237

FSP -238

FSP -239

FSP -240

FSP-241

FSP -242

FSP-2 43

FSP-244

FSP -245

FSP-246

FSP -247

FSP-248

FSP-249

FSP -250

FSP -251

FSP-252

FSP-253

FSP-254

FSP-255

FSP -256

FSP -257

FSP-258

FSP-259

FSP -260

FSP-261

FSP-262

FSP -263

FSP-264

FSP -265

FSP-266

FSP-267

FSP -268

FSP-269

FSP -270

FSP-271

FSP -272

FSP-273

FSP-274

FSP-275

FSP -276

FSP-277

FSP -278

FSP -279

FSP-280

FSP-281

FSP-282

FSP -283

FSP -284

FSP -285
$5.4 \quad 16$

5.7

5.5

5.2

5.7

5
4.8

4.7

5.2

4.8

4.6

5.7

5.4

4.7

4.9

4.4

4.6

5.2

5.3

4.6

4.4

5.2

5.1

4.8

5.2

4.5

5.1

5.4

5.4

4.9

4.9

4.4

4.8

4

3.8

3.7

4.1

4

3.2

3. 1

3

3.1

4.6

3.9

4.1

4

4.5

4.3

4.9

4.2

4.9
WSRC-RP-90-0591

$803 / 29 / 89$

$1003 / 29 / 89$

$803 / 29 / 89$

$1103 / 30 / 89$

$1203 / 30 / 89$

$603 / 30 / 89$

$403 / 30 / 89$

$803 / 30 / 89$

$203 / 30 / 89$

$803 / 30 / 89$

$603 / 30 / 89$

$703 / 30 / 89$

$403 / 30 / 89$

$503 / 30 / 89$

$10 \quad 03 / 30 / 89$

$803 / 30 / 89$

$903 / 30 / 89$

$403 / 30 / 89$

$503 / 30 / 89$

$503 / 30 / 89$

$1203 / 30 / 89$

$403 / 30 / 89$

$603 / 30 / 89$

$403 / 30 / 89$

$803 / 30 / 89$

$803 / 30 / 89$

$903 / 30 / 89$

$203 / 31 / 89$

$803 / 31 / 89$

$603 / 31 / 89$

$403 / 31 / 89$

$803 / 31 / 89$

$203 / 31 / 89$

$204 / 11 / 89$

$304 / 11 / 89$

$604 / 11 / 89$

$404 / 11 / 89$

surface $04 / 11 / 89$

$504 / 11 / 89$

surface $04 / 11 / 89$

$804 / 11 / 89$

$1004 / 11 / 89$

$804 / 11 / 89$

$704 / 12 / 89$

surface $04 / 12 / 89$

$904 / 12 / 89$

surface $04 / 12 / 89$

$304 / 12 / 89$

$604 / 12 / 89$

$704 / 12 / 89$

$\begin{array}{lr}14 & 04 / 12 / 89 \\ 14 & \text { surface } 04 / 12 / 89\end{array}$ 
Appendix C

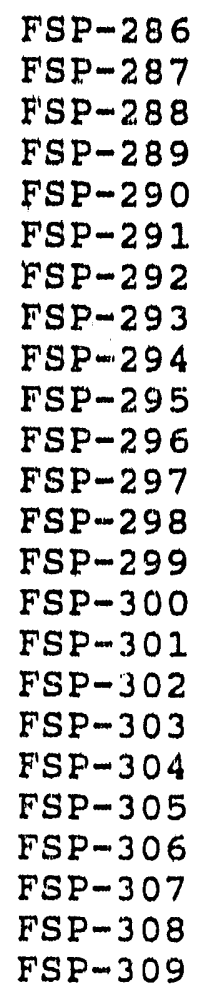

4.1

4.4

3.6

3.7

3.6

4.2

4.5

4.2

4.1

4

4.5

5.2

4.4

4.2

4.3

4.6

4.5

5.4

4.4

4.5

4.2

4.1

4.6

4
WSRC-RP-90-0591

$\begin{array}{lrr}14 & 6 & 04 / 12 / 89 \\ 14 & 3 & 04 / 12 / 89 \\ 13 & 5 & 04 / 13 / 89 \\ 13 & 4 & 04 / 13 / 89 \\ 13 & 4 & 04 / 13 / 89 \\ 13 & 5 & 04 / 13 / 89 \\ 13 & 8 & 04 / 13 / 89 \\ 13 & 9 & 04 / 13 / 89 \\ 13 & 4 & 04 / 13 / 89 \\ 13 & 6 & 04 / 13 / 89 \\ 14 & \text { surface } & 04 / 13 / 89 \\ 14 & 7 & 04 / 13 / 89 \\ 14 & \text { surface } & 04 / 13 / 89 \\ 14 & 3 & 04 / 13 / 89 \\ 14 & 4 & 04 / 13 / 89 \\ 14 & 8 & 04 / 13 / 89 \\ 14 & \text { surface } & 04 / 13 / 89 \\ 14 & \text { surface } & 04 / 13 / 89 \\ 14 & 4 & 04 / 13 / 89 \\ 14 & 1 & 04 / 13 / 89 \\ 14 & 1 & 04 / 13 / 89 \\ 14 & 5 & 04 / 13 / 89 \\ 14 & 3 & 04 / 13 / 89 \\ 14 & 4 & 04 / 13 / 89\end{array}$




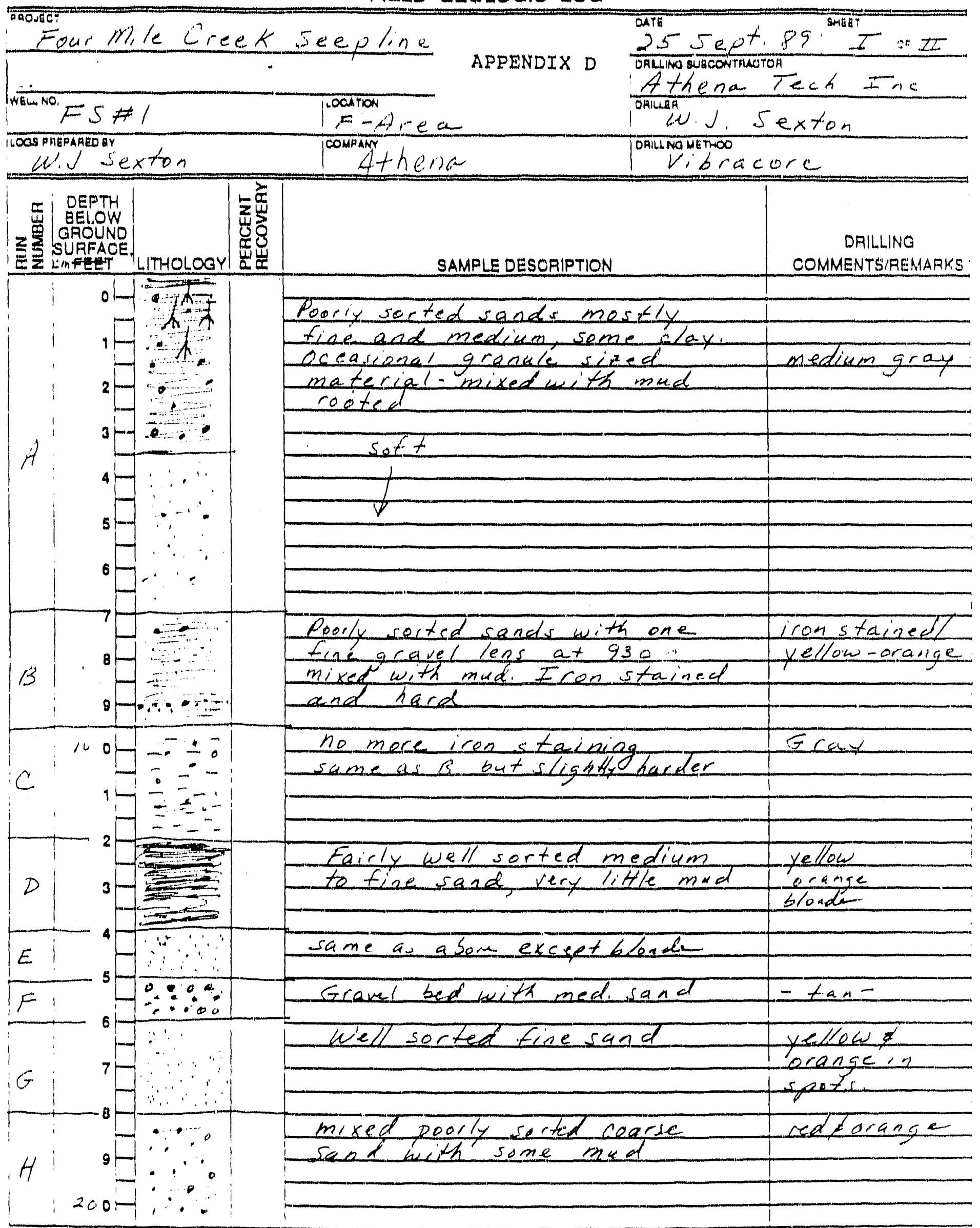


OAOJET Four mile ireek seepline

$$
\text { FS FI }
$$

LOOS PAEPARED OY

$$
\text { W. J. Sexton }
$$

\section{WDCATION}

F-Area

compan
$125.5 e p t .89 ! 20 \pi$

DALINO SUPCONTALOTOR

Athena Tech Inc W.J. Sexton

OHILNOWETROO Vibracore

(1)

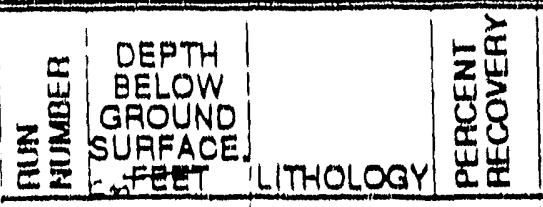

SAMPLE DESCAIPTION

DAILLING

COMMENTS/REMARKS

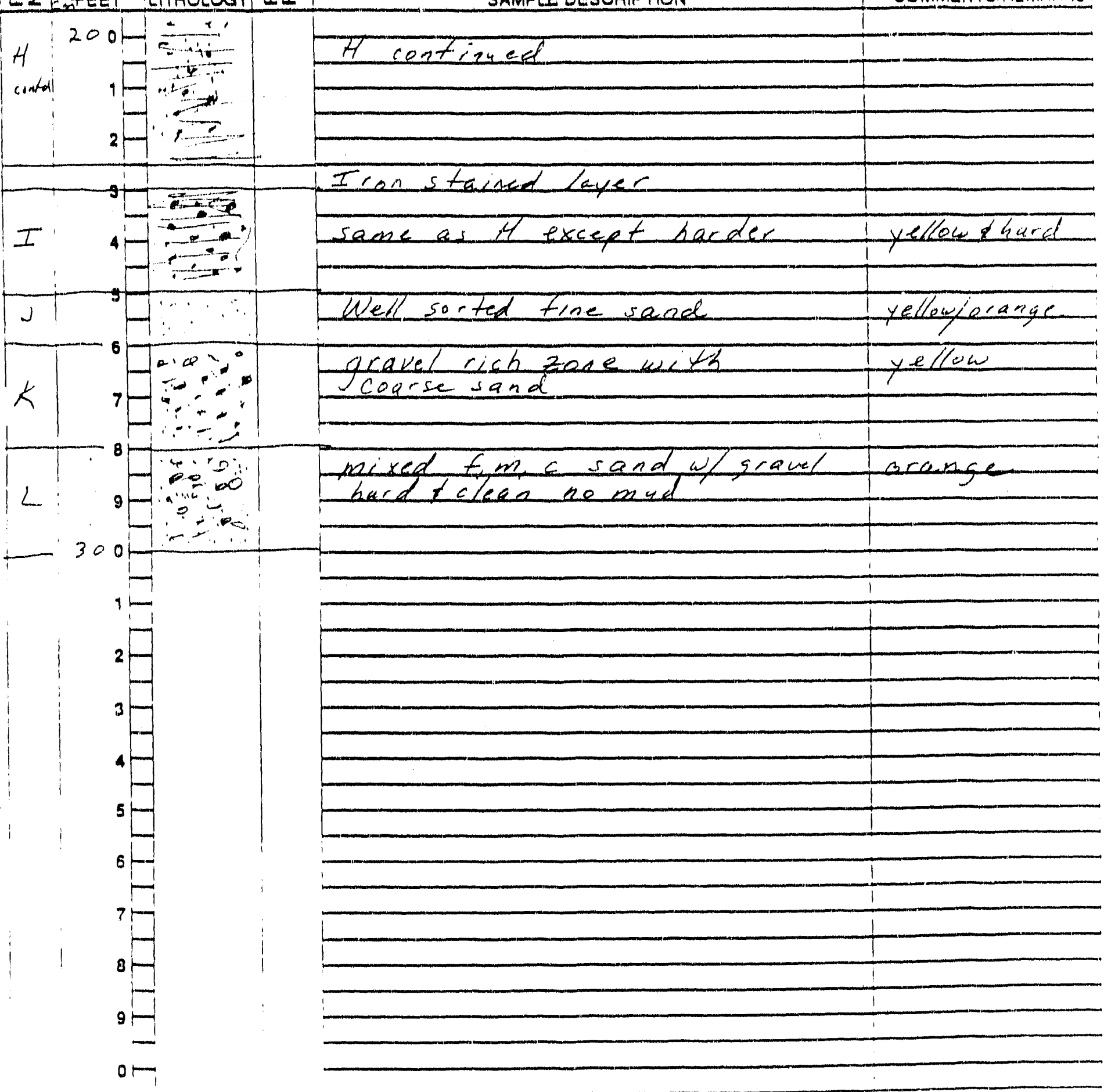




\begin{tabular}{|c|c|c|}
\hline Four mile Creek & Seepline & 25 sept. $851^{21} I$ or II \\
\hline - & & 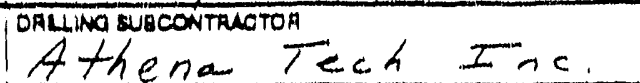 \\
\hline$=5 \neq 2$ & Fochtow & WR.J. Sexton \\
\hline $\begin{array}{l}\text { WOAS PAEPARED OY } \\
\text { W.S.SETOA }\end{array}$ & consum 4 thena & DARLLMOMETHOO \\
\hline
\end{tabular}

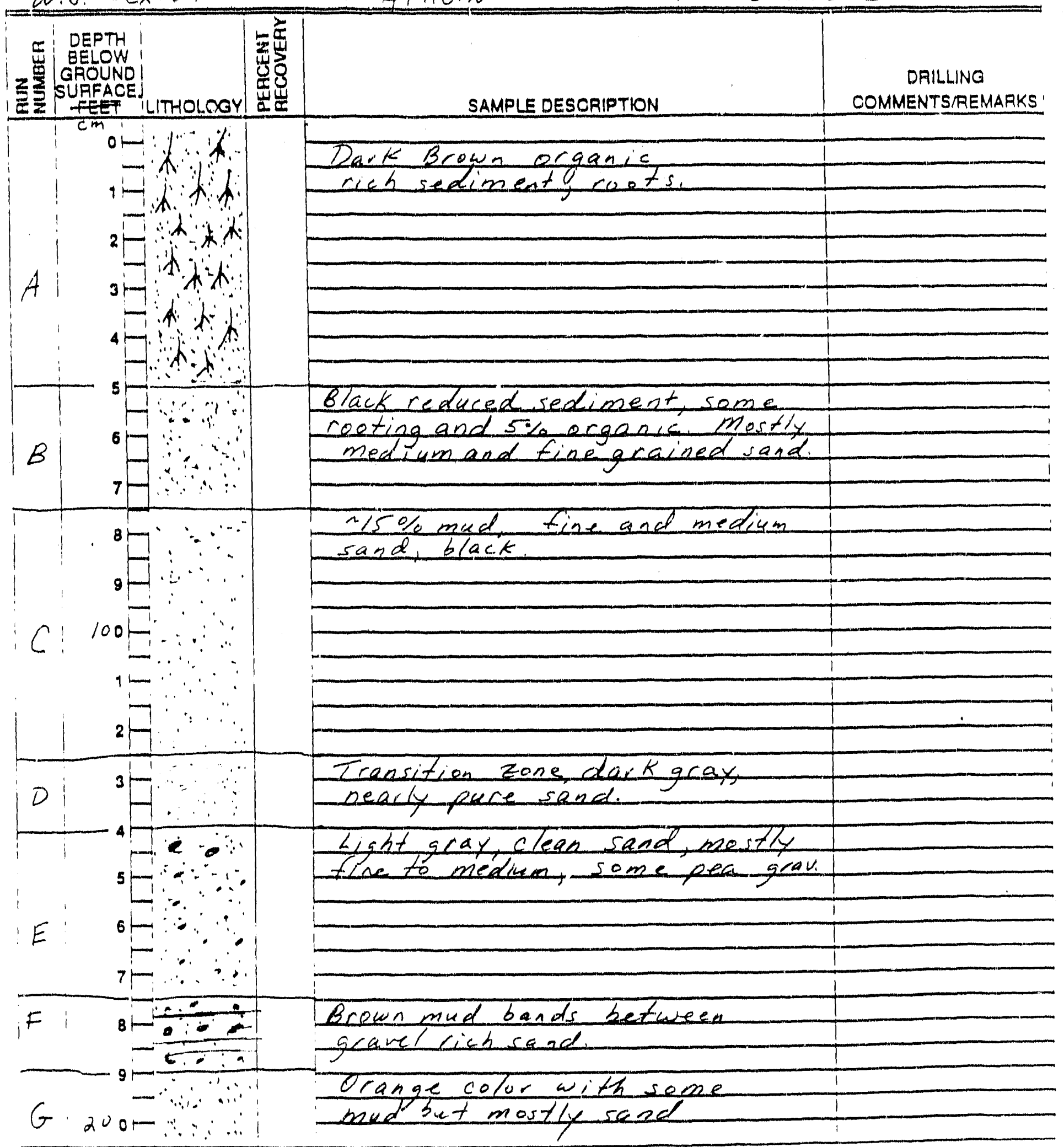




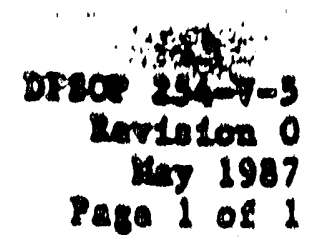

cinn

mID GEOLOAC lOO

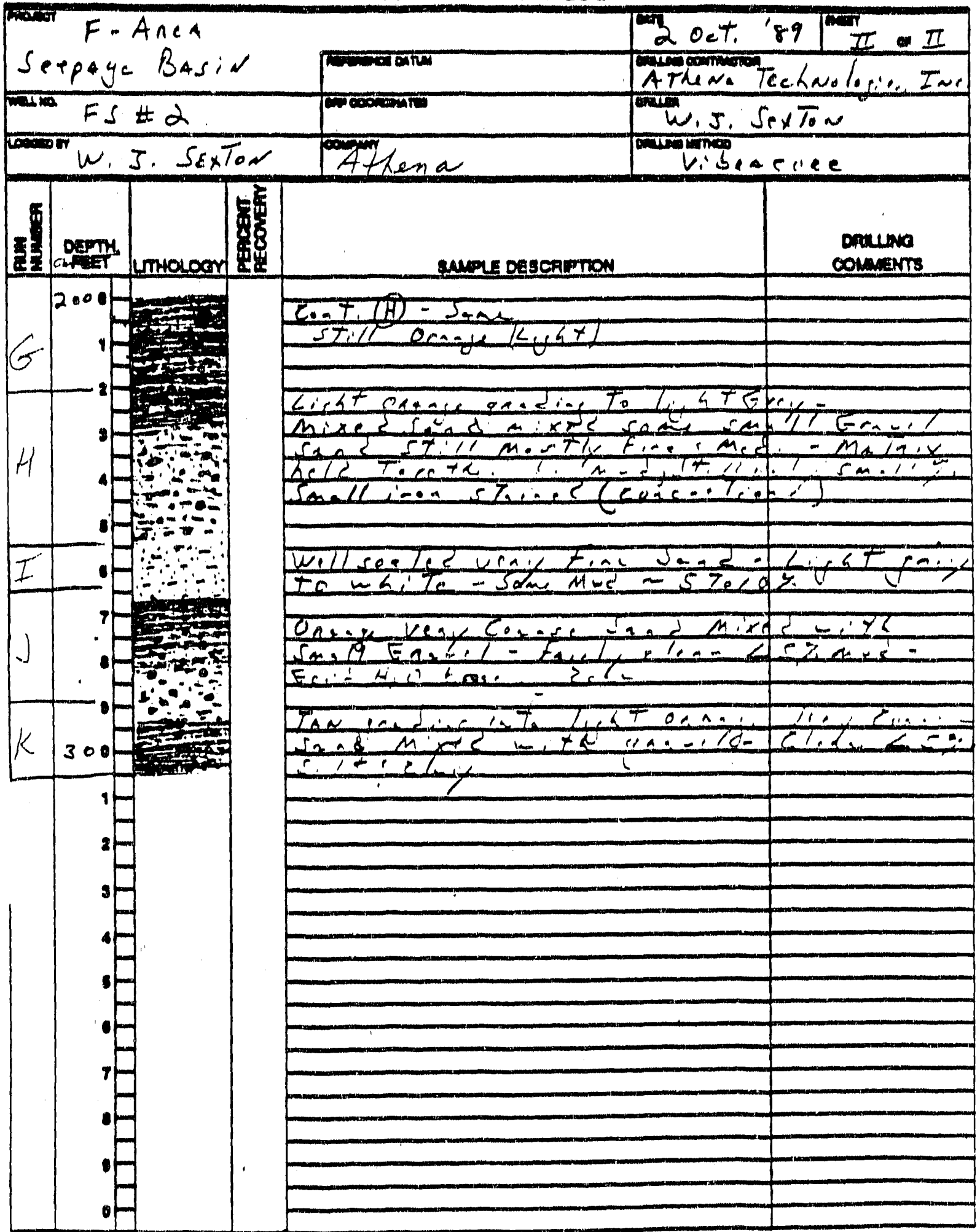




\section{क्वसहर}

Four mile Ureek Seepline

...

NES NO.

$$
\text { Fs } 3
$$

LOGS PAEPAREC BY

W.J. Sexton

$$
\begin{aligned}
& \text { Fochion } \\
& \text { comprea } \\
& 4 \text { thena }
\end{aligned}
$$
ORULER

W.J. Sexton

\section{$=$ DEPTH BELOW GROUND

$$
0
$$
- 在 $x^{-4}-4$

$1 \frac{1}{1 ! 2}=$

B $\quad 2$

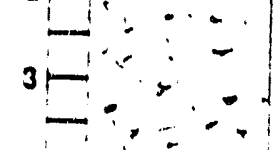

(c)

$4-\frac{1}{2-0 .}$

T

50

$6+2$

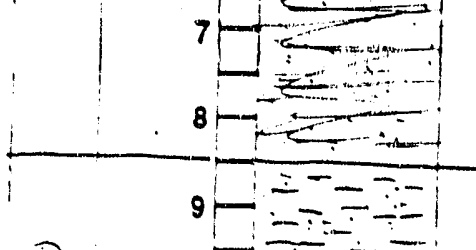

D

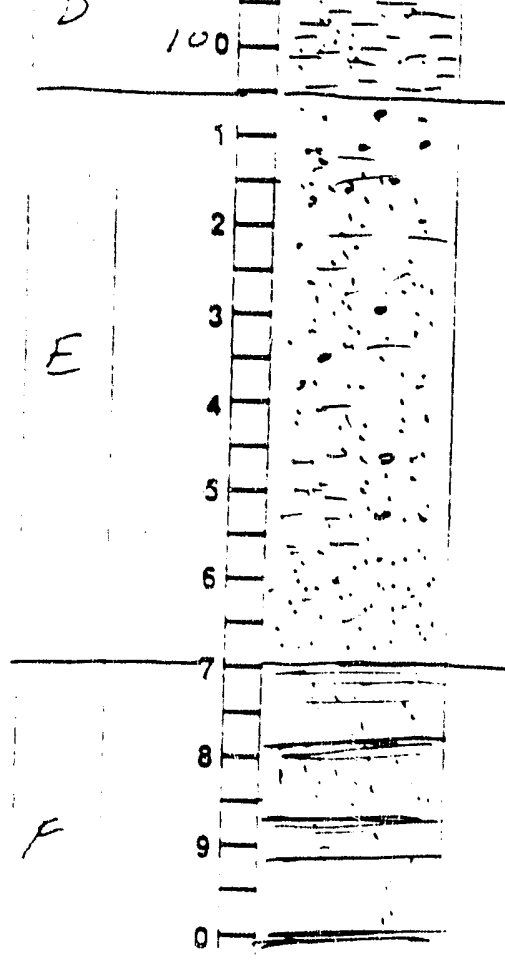

SAMPLEDESCRIPTION Vibracore

DRILLING COMMENTSIREMARKS fine to medium sand with reets and mad

mediun to coarse sandsome oxidation, orange

\section{mixed fine and medium \\ and organics}

Tlne sand with high mad
content, light guak

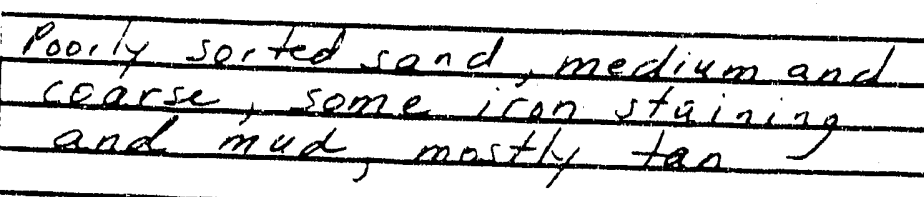

Fine sand witi occaisonal mediug and coarse sand, 


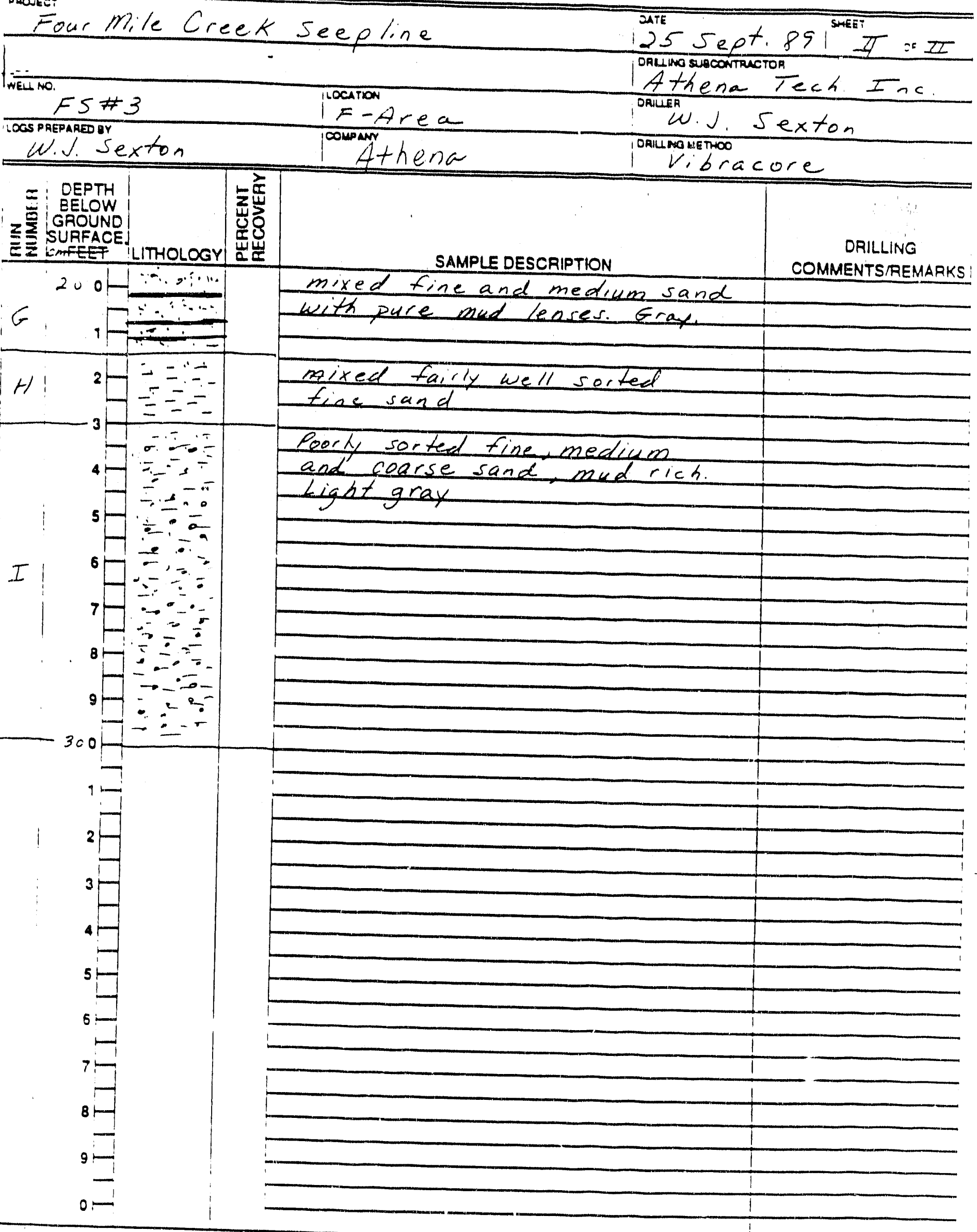




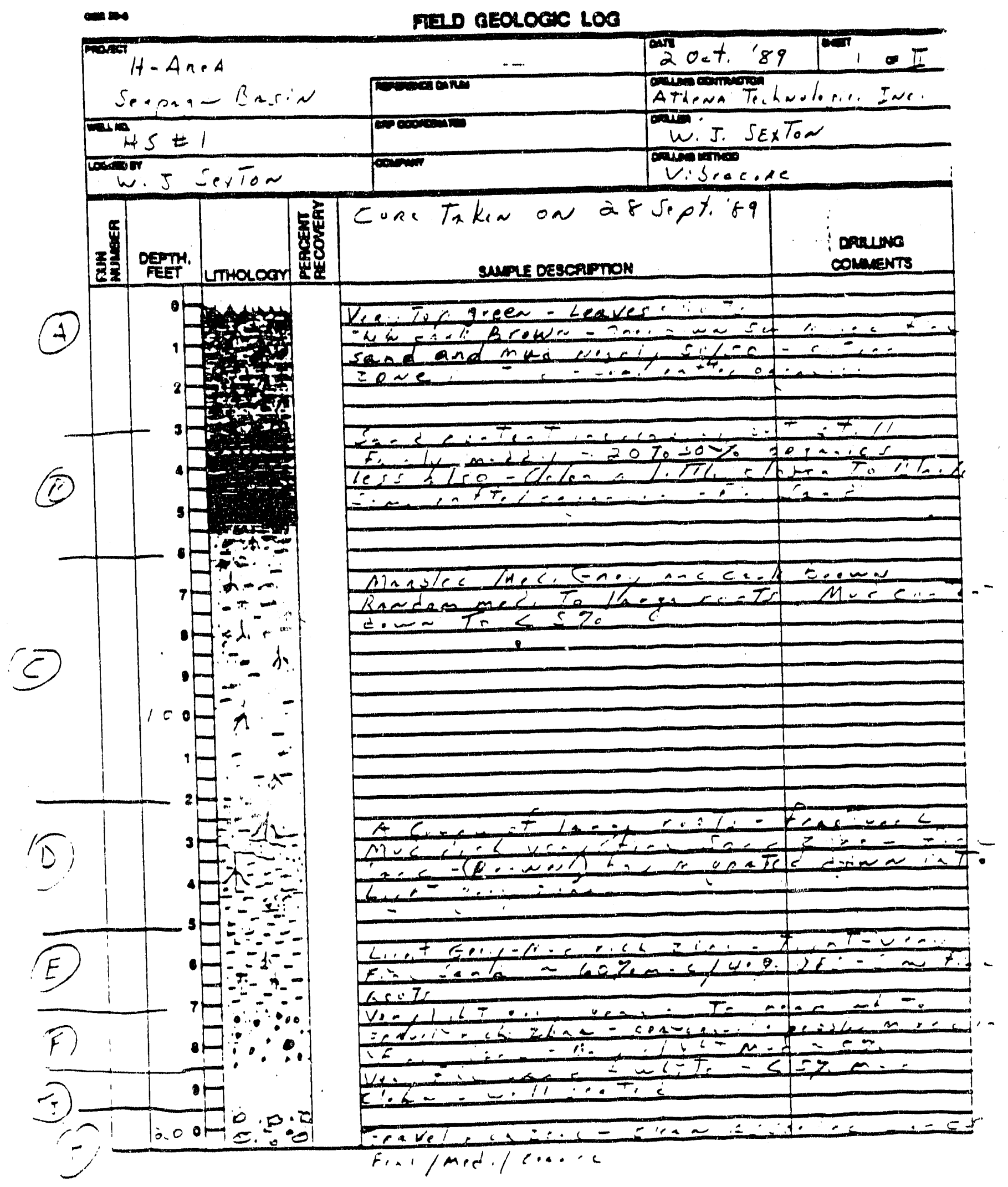




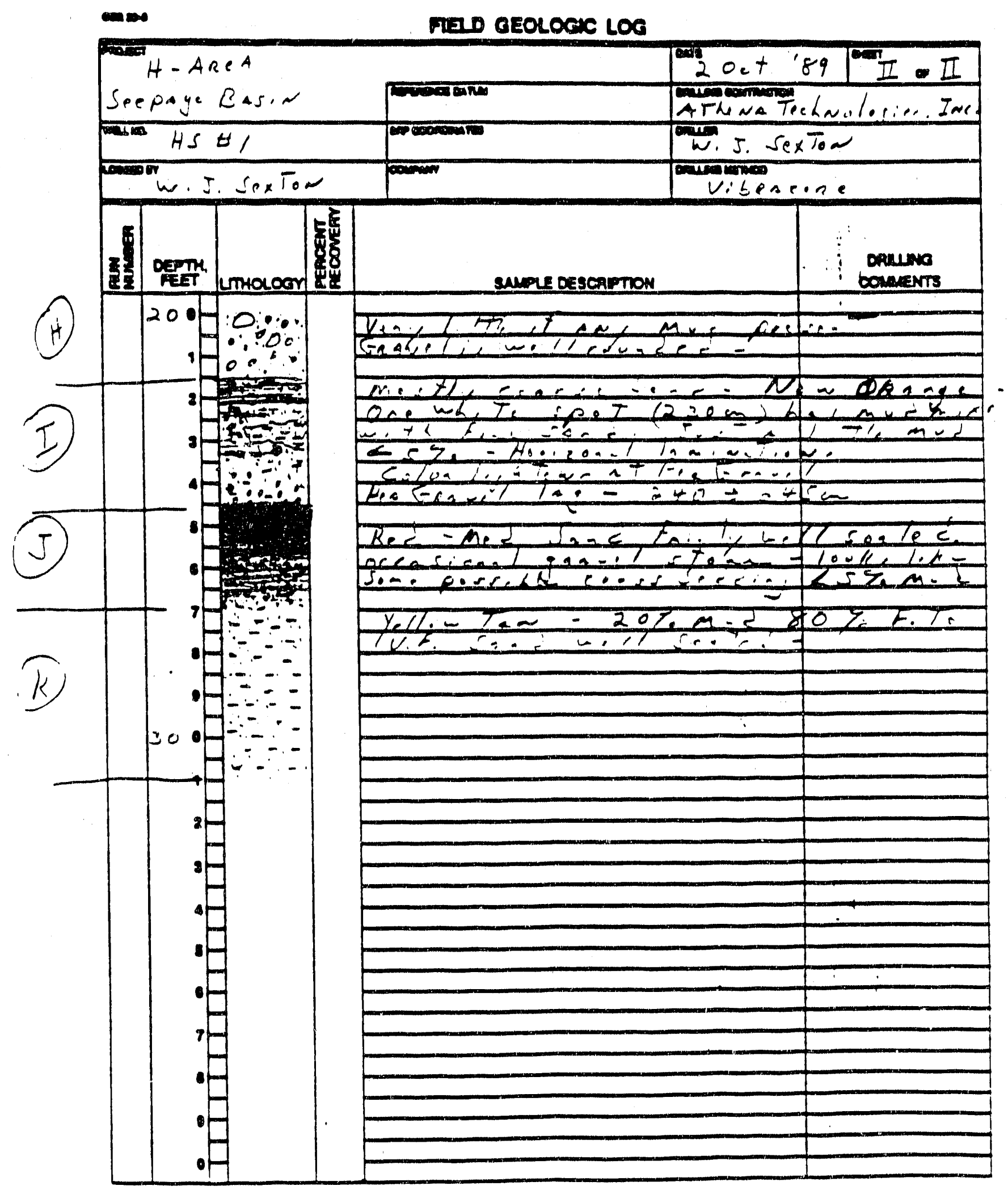




\title{
DAOJEC
}

Four mile Creek Sizepline

$i^{\text {WELLNO. }}$ HS 2

WOGS PAEPAAED QY

W.S.Sexton

\author{
LOCATION \\ H-Area \\ COMPAMY \\ Athena
}

20 Sept. $\left.89\right|_{\text {ISTEET }} ^{\text {SAE of II }}$ Aruino sugcontractor Tech. Inc. DRILER. S Sexton
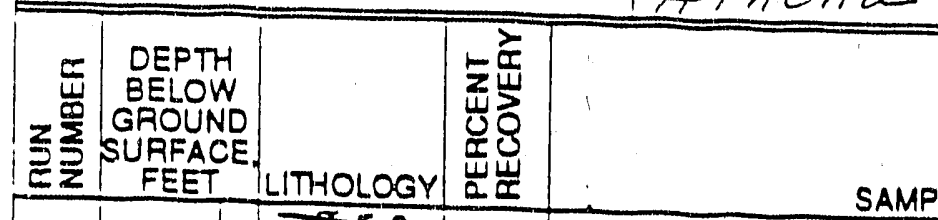

DAILNO METHOO Vibracore

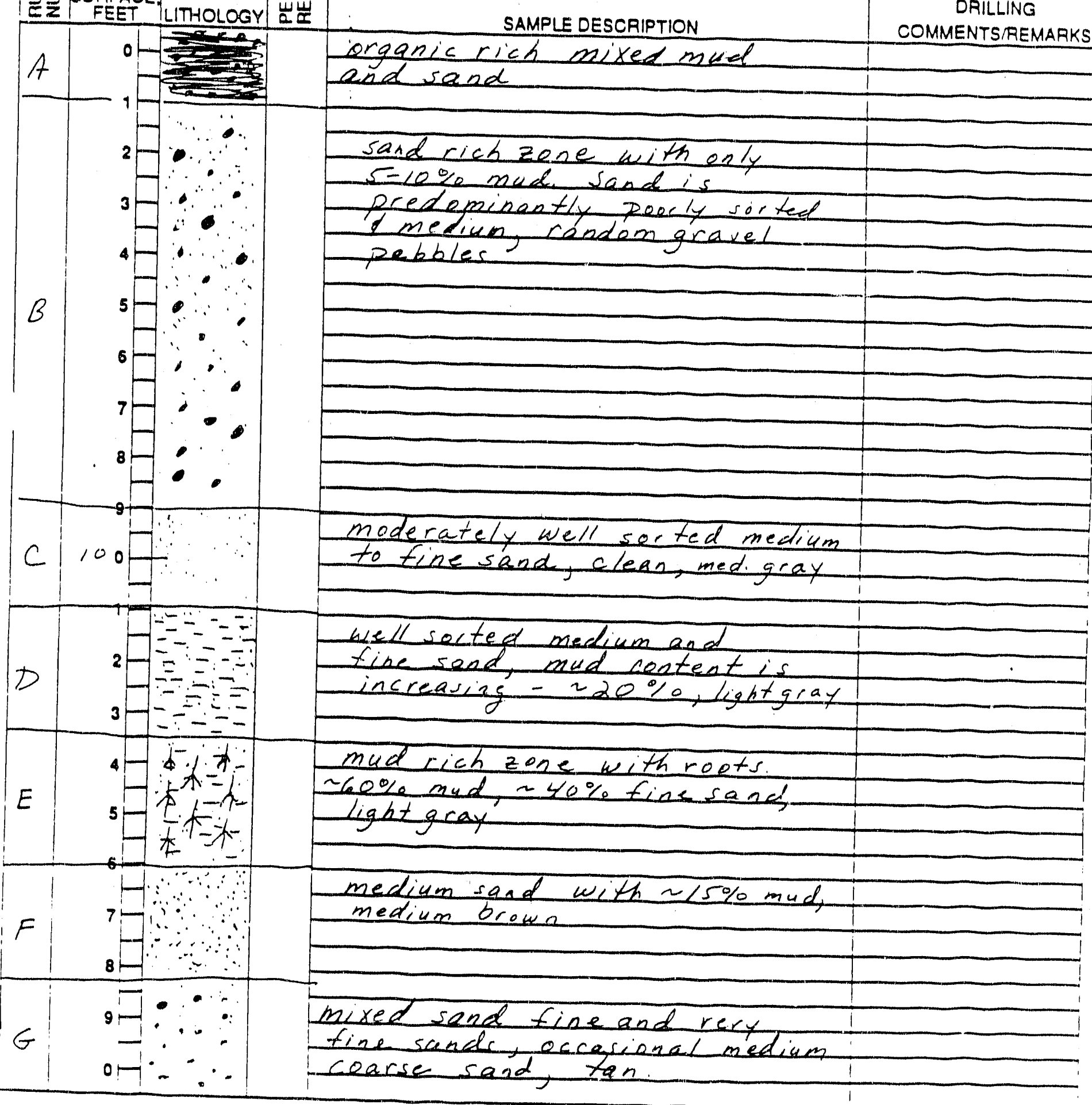




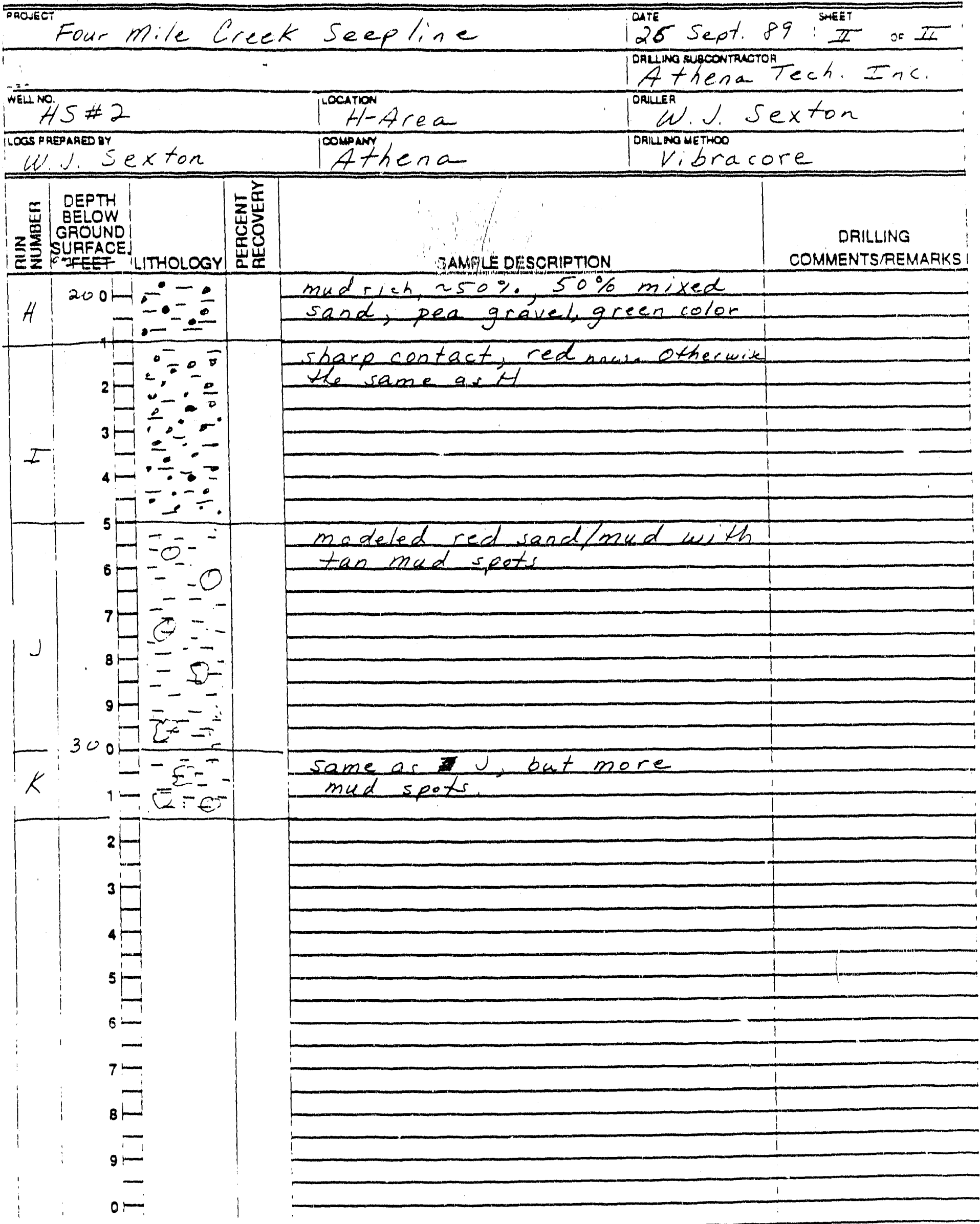


To: Carol Cunnins, SRS

Dan Rogers, SRS.

From: Lisa Vogel. $\mathrm{T} / \mathrm{b}$

Re: Drinking Water Standards

Date: January 23, 1990

I have compiled the drinking water standards for the Annual Report. The EPA proposed standards for radionuclides in 1986 and for some miscellaneous ana. lytes in May of 1989. Neither the 1986 nor the 1989 proposals have been accepted. We have decided to use all the established standards we have and to use proposed standards for any analytes that do not have established stand. ards. Please let me know whether or not you agree with this decision. Once I have received approval, I will be able to complete the Drinking Water Stand. ards table in just a day or so.

CFR, 1986 gives a maximum contaminant leve! Eor turbidity. This level is applicable only to drinking water systems that use surface water; therefore, we have decided to omit turbidity from our list of drinking water standards.

The maximum contaminant level for cesium-134 in EPA, 1977, appears to be a mistake. Therefore, we have decided to use the proposed level from EPA, 1986 , for cesium-134.

The following table compares the proposals with the established standards.

\begin{tabular}{|c|c|c|c|c|}
\hline Analvce & $\begin{array}{l}\text { Established } \\
\text { Standard }\end{array}$ & Source & $\begin{array}{l}\text { Proposed } \\
\text { Standard }\end{array}$ & Source \\
\hline Arsenic & $0.05 \mathrm{mg} / \mathrm{L}$ & CFR, 1987 & - & - \\
\hline Barium & $1 \mathrm{mg} / \mathrm{L}$ & CFR, 1987 & $5 \mathrm{mg} / \mathrm{L}$ & EPA, 1989 \\
\hline Cadmium & $0.01 \mathrm{mg} / \mathrm{L}$ & CFR, 1987 & $0.005 \mathrm{mg} / \mathrm{L}$ & EPA, 1989 \\
\hline Chromiura & $0.05 \mathrm{mg} / \mathrm{L}$ & CFR, 1987 & $0.1 \mathrm{mg} / \mathrm{L}$ & EPA, 1989 \\
\hline Lead & $0.05 \mathrm{mg} / \mathrm{L}$ & CFR， 1987 & - & - \\
\hline Mercury & $0.002 \mathrm{mg} / \mathrm{L}$ & CFR, 1987 & - & - \\
\hline Nitrate (as N) & $10 \mathrm{mg} / \mathrm{L}$ & CFR, 1987 & - & - \\
\hline Seleniurn & $0.01 \mathrm{mg} / \mathrm{L}$ & CFR, 1987 & $0.05 \mathrm{mg} / \mathrm{L}$ & EPA, 1989 \\
\hline Silver ! ! & $0.05 \mathrm{mg} / \mathrm{L}$ & CFR, 1987 & - & \\
\hline Gross alpha & $15 \mathrm{pCi} / \mathrm{L}$ & CFR, 1987 & $15 \mathrm{pCi} / \mathrm{L}$ & EPA, 1986 \\
\hline Tritium & $20 \mathrm{pCi} / \mathrm{mL}$ & CFR, 1987 & $90 \mathrm{pCi} / \mathrm{mL}$ & EPA, 1986 \\
\hline Endrin & $0.0002 \mathrm{mg} / \mathrm{L}$ & CFR, 1987 & & $\cdot$ \\
\hline Lindane & $0.004 \mathrm{mg} / \mathrm{L}$ & CFR, 1987 & $0.0002 \mathrm{mg} / \mathrm{L}$ & EPA, 1989 \\
\hline Methoxyctilor & $0.1 \mathrm{mg} / \mathrm{L}$ & CFR, 1987 & $0.4 \mathrm{mg} / \mathrm{L}$ & EPA, 1989 \\
\hline Toxapherie & $0.005 \mathrm{mg} / \mathrm{L}$ & CFR, 1987 & - & - \\
\hline $2,4 \cdot D$ & $0.1 \mathrm{mg} / \mathrm{L}$ & CFR, & $0.07 \mathrm{mg} / \mathrm{L}$ & EPA, 1989 \\
\hline silvex & $0.01 \mathrm{mg} / \mathrm{L}$ & CFR, 1987 & $0.05 \mathrm{mg} / \mathrm{L}$ & EPA, 1989 \\
\hline Chlorof am: ax & $0.1 \mathrm{mg} / \mathrm{L}$ & CFR, 1987 & - & - \\
\hline Fluoride & $4 \mathrm{mg} / \mathrm{L}$ & CFR, 1986 & - & - \\
\hline
\end{tabular}




Analyte
1,4-Dichlorobenzene
(p-Dichlorobenzene)
Benzene
Carbon tetrachloride
1,2-Dichloroethane
Trichloroethene
Vinyl chloride
(Chloroethene)
1,1-Dichloroethene
1,1, Irichloroethane
Berylilum-7
Carbon-14
Chromium-51
Manganese-54
Iron-55
Iron-59
Cobalt-58
Cobalt-60
Nickel-59
Nickel-63
Zinc-65
Strontium-89
Strontium-90
Zirconium-95
Technetium-99
Ruthenium-103
Ruthenium-106
Antimony-125
Iodine-129
Iodine-131
Cesium-134
Cesium-137
Barium-140
Radium-226
Radium-228
Radium-226+228
Nitrite (as N)
Chlordane
Dibromochloropropane
cis-1,2-Dichloro-
ethene
trans-1,2-Dichloro-
ethene
1,2-Dichloropropane
Ethylbenzene
Heptachlor
Heptachlor epoxide
PCB's
Pentachloropheno1
Styrene
Tetrachloroethene

Established Standard

$0.075 \mathrm{mg} / \mathrm{L}$

$0.005 \mathrm{mg} / \mathrm{L}$

$0.005 \mathrm{mg} / \mathrm{L}$

$0.005 \mathrm{mg} / \mathrm{L}$

$0.005 \mathrm{mg} / \mathrm{L}$

$0.002 \mathrm{mg} / \mathrm{L}$

$0.007 \mathrm{mg} / \mathrm{L}$

$0.2 \mathrm{mg} / \mathrm{L}$

$6,000 \mathrm{pC1} / \mathrm{L}$

$2,000 \mathrm{pCl} / \mathrm{L}$

$6,000 \mathrm{pCi} / \mathrm{L}$

$300 \mathrm{pCi} / \mathrm{L}$

2,000 pCi/L

$200 \mathrm{pCi} / \mathrm{L}$

$9,000 \mathrm{pCi} / \mathrm{L}$

$100 \mathrm{pC1} / \mathrm{L}$

$300 \mathrm{pCi} / \mathrm{L}$

$50 \mathrm{pCl} / \mathrm{L}$

$300 \mathrm{pC1} / \mathrm{L}$

$20 \mathrm{pCi} / \mathrm{L} * *$

$8 \mathrm{pCi} / \mathrm{L}$

$200 \mathrm{pCi} / \mathrm{L}$

$900 \mathrm{pCl} / \mathrm{L}$

$200 \mathrm{pCi} / \mathrm{L}$

$30 \mathrm{pCi} / \mathrm{L}$

$300 \mathrm{pCi} / \mathrm{L}$

$1 \mathrm{pCi} / \mathrm{L}$

$3 \mathrm{pCi} / \mathrm{L}$

$20,000 \mathrm{pCi} / \mathrm{L}$

$200 \mathrm{pCi} / \mathrm{L}$

$90 \mathrm{pCi} / \mathrm{L}$

$5 \mathrm{pCi} / \mathrm{L}$

Source

EPA, 1987

EPA, 1987

EPA, 1987

EPA, 1987

EPA, 1987

EPA, 1987

EPA, 1987

EPA, 1987

EPA, 1977

EPA, 1977

EPA, 1977

EPA, 1977

EPA, 1977

EPA, 1977

EPA, 1977

EPA, 1977

EPA, 1977

EPA, 1977

EPA, 1977

EPA, 1977

EPA, 1977

EPA, 1977

EPA, 1977

EPA, 1977

EPA, 1977

EPA, 1977

EPA, 1977

EPA, 1977

EPA, 1977

EPA, 1977

EPA, 1977

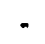

CFR, 1987
Proposed

Standard

Soute

100,000 oCt/1

$100,000 \mathrm{pCi} / \mathrm{L} \quad$ EPA, 1986

$3,000 \mathrm{pCI} / \mathrm{L} \quad \mathrm{EPA}, 1986$

$80,000 \mathrm{pCi} / \mathrm{L} \quad \mathrm{EPA}, 1986$

3,000 pCi/L EPA, 1986

$10,000 \mathrm{pCi} / \mathrm{L} \quad$ EPA, 1986

$1,000 \mathrm{pCi} / \mathrm{L}$

$2,000 \mathrm{pCi} / \mathrm{L}$

$200 \mathrm{pCi} / \mathrm{L}$

$30,000 \mathrm{pCi} / \mathrm{L}$

$10,000 \mathrm{pCi} / \mathrm{L}$

$400 \mathrm{pCi} / \mathrm{L}$

$900 \mathrm{pCi} / \mathrm{L}$

$50 \mathrm{pCi} / \mathrm{L}$

$3,000 \mathrm{pCi} / \mathrm{L}$

$5,000 \mathrm{pCi} / \mathrm{L}$

4,000 pCi/h

$300 \mathrm{pCl} / \mathrm{L}$

4,000 pCi/L

$100 \mathrm{pCi} / \mathrm{L}$

$700 \mathrm{pCl} / \mathrm{L}$

$80 \mathrm{pCi} / \mathrm{L}$

$100 \mathrm{pCi} / \mathrm{L}$

$1,000 \mathrm{pCi} / \mathrm{L}$

$4 \mathrm{pCl} / \mathrm{L}$

$8 \mathrm{pCi} / \mathrm{L}$

$0.001 \mathrm{mg} / \mathrm{L}$

$0.002 \mathrm{mg} / \mathrm{L}$

$0.0002 \mathrm{mg} / \mathrm{L}$

$0.07 \mathrm{mg} / \mathrm{L}$

$0.1 \mathrm{mg} / \mathrm{L}$

$0.005 \mathrm{mg} / \mathrm{L}$

$0.7 \mathrm{mg} / \mathrm{L}$

$0.0004 \mathrm{mg} / \mathrm{L}$

$0.0002 \mathrm{mg} / \mathrm{L}$

$0.0005 \mathrm{mg} / \mathrm{L}$

$0.2 \mathrm{mg} / \mathrm{L}$

$0.005 \mathrm{mg} / \mathrm{L}$

$0.005 \mathrm{mg} / \mathrm{L}$
EPA, 1986

EPA, 1986

EPA, 1986

EPA, 1986

EPA, 1986

EPA, 1986

EPA, 1986

EPA, 1986

EPA, 1986

EPA, 1986

EPA, 1986

EPA, 1986

EPA, 1986

EPA, 1986

EPA, 1986

EPA, 1986

EPA, 1986

EPA, 1986

EPA, 1986

EPA, 1986

EPA, 1989

EPA, 1989

EPA, 1989

EPA, 1989

EPA, 1989

EPA. 1989

EPA, 1989

EPA, 1.989

EPA, 1989

EPA, 1989

EPA, 1989

EPA, 1989

E.PA, 1989 


\begin{tabular}{|c|c|c|c|c|}
\hline nalyten & $\begin{array}{l}\text { Established } \\
\text { Standard }\end{array}$ & Source & $\begin{array}{l}\text { Proposed } \\
\text { Standard }\end{array}$ & Sonlence \\
\hline Toluene: & - & $\cdot$ & $2 \mathrm{mg} / \mathrm{L}$ & EPA, 1989 \\
\hline Kylenr:s & - & - & $10 \mathrm{mg} / \mathrm{L}$ & EPA, 1989 \\
\hline Nonvolatile beta & . & - & $50 \mathrm{pCi} / \mathrm{L}$ & EPA, 1986 \\
\hline Potassium-40 & - & - & $300 \mathrm{pCi} / \mathrm{L}$ & EPA, 1986 \\
\hline $\operatorname{Tin} \cdot 113$ & - & - & $4,000 \mathrm{pCl} / \mathrm{L}$ & EPA, 1986 \\
\hline Urantum-23/4 & - & $\cdot$ & $28 \mathrm{pCl} / \mathrm{L}$ & EPA, 1986 \\
\hline Uranium-235 & $\cdot$ & $\cdot$ & $28 \mathrm{pCl} / \mathrm{L}$ & EPA, 1986 \\
\hline Uranium-238 & $\cdot$ & $\cdot$ & $28 \mathrm{pCl} / \mathrm{L}$ & EPA, 1986 \\
\hline Americium-241 & $\cdot$ & $\cdot$ & $4 \mathrm{pC1} / \mathrm{L}$ & $E P A, 1986$ \\
\hline
\end{tabular}

* The level for cotal trihalomethanes is set at $0.1 \mathrm{mg} / \mathrm{L}$. Because bromated methanes are rarely detected in SRS groundwater. EHP presumes that most of the trihalomethanes present in plant water are chloroform.

** This is the lower of two levels given for strontium-89.

Sources:

CFR (Code of Federal Regulations), 1986. "National Primary Drinking Water Regulations," 40 CFR, Part 141, Pp, 521-568, Washington, DC.

CFR (Code of Federal Regulations), 1987. "National Primary Drinking water Regulations," 40 CFR, Part 141, PP. 526-575, Washington, DC.

EPA (U.S. Environmental Protection Agency), 1977. National Interim Primary Drinking Water Regulations, EPA-570/9-76-003, Washington, DC.

EPA (U.S. Environmental Protection Agency), 1986. "Water Pollution Control National Primary Drinking Water Regulations, Radionuclides (Proposed)," Federal Register, September 30, 1986, pp. 34836-34862, Washington, DC.

EPA (U.S. Environmental Protection Agency), 1987. "National Primary Drinking Water Regulations: Synthetic Organic Chemicals; Monicoring for Unregulated Contaminants," Federal Register, July 8, 1987, pp. 25690-25717, Washing. ton, DC.

EPA (U.S. Environmental Protection Agency), 1989. "National Primary and Secondary Drinking Water Regulations (Proposed Rule), "Federal Register, May 22, 1989, PP. 22062-2216\%, Washington, DC. 

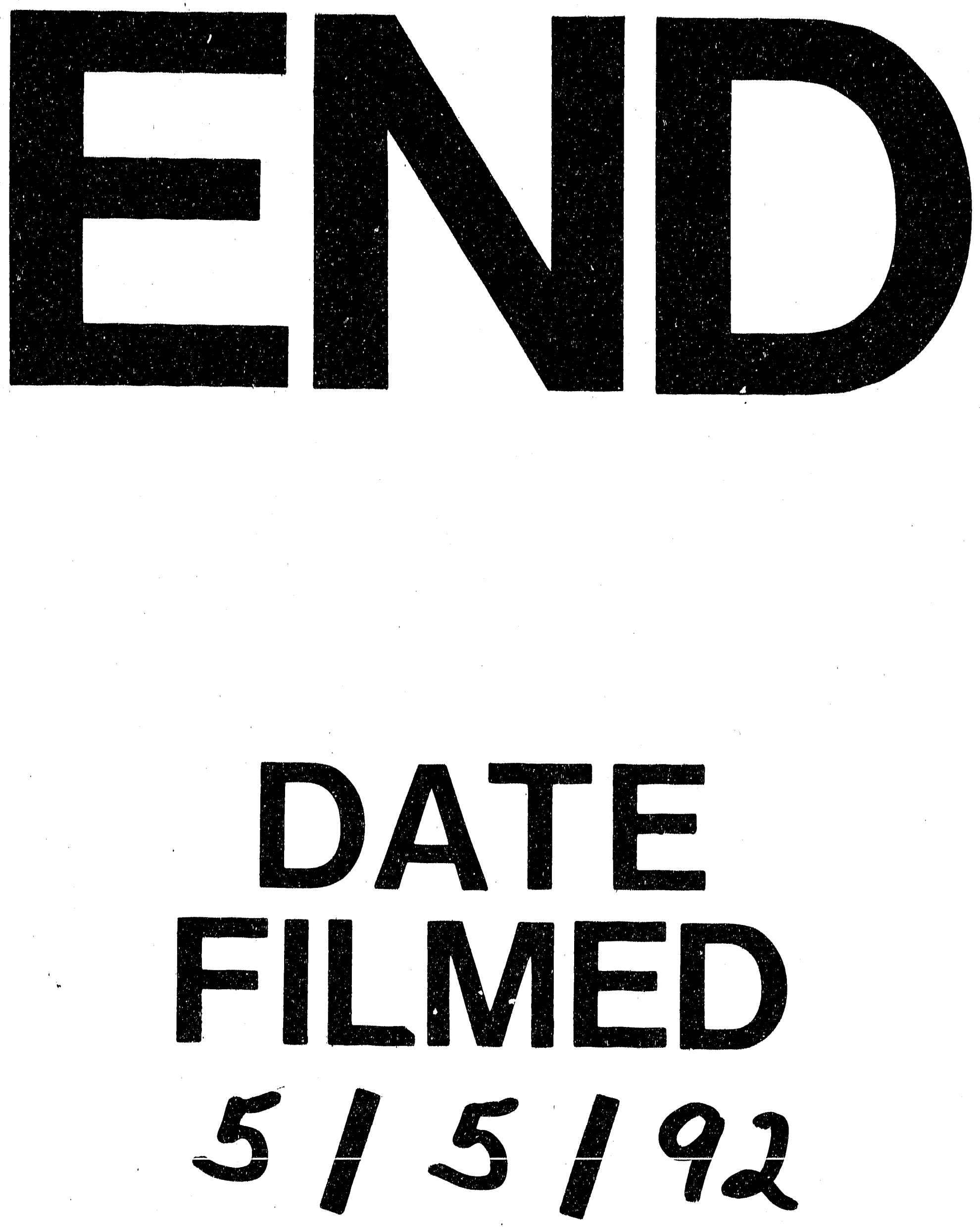
